Federal Reserve Bank of Minneapolis

Research Department Staff Report 373

May 2006

\title{
Asset Prices and Liquidity in an Exchange Economy
}

\author{
Ricardo Lagos* \\ Federal Reserve Bank of Minneapolis \\ and New York University
}

\begin{abstract}
I develop an asset-pricing model in which financial assets are valued for their liquidity - the extent to which they are useful in facilitating exchange - as well as for being claims to streams of consumption goods. The implications for average asset returns, the equity-premium puzzle and the risk-free rate puzzle, are explored in a version of the model that nests the work of Mehra and Prescott (1985).
\end{abstract}

*First draft: May 2005. I am indebted to Ellen McGrattan and Fabrizio Perri for many helpful conversations at various stages. I am grateful to Nobu Kiyotaki for his feedback as a discussant at the Princeton-NY Fed Conference on Liquidity and the Philadelphia Workshop on Monetary and Macroeconomics. I thank V.V. Chari, Mark Gertler, Patrick Kehoe, Narayana Kocherlakota, Hanno Lustig, Erzo G. J. Luttmer, Monika Piazzesi, Ed Prescott, Guillaume Rocheteau, Tom Sargent, Martin Schneider, Pierre-Olivier Weill, Michael Woodford, and Randy Wright for their input. I also thank Katya Kartashova for many useful comments on previous drafts. Daniil Manaenkov, Carlos Serrano, and Jing Zhang provided research assistance. Financial support from the C.V. Starr Center for Applied Economics at NYU is gratefully acknowledged. The views expressed herein are those of the author and not necessarily those of the Federal Reserve Bank of Minneapolis or the Federal Reserve System. 


\section{Introduction}

In this paper I develop an asset-pricing model in which financial assets are valued not only as claims to streams of consumption goods but also for their liquidity. By liquidity I mean the degree to which an asset is valued as a medium of exchange at the margin. Specifically, I study a class of exchange economies in which agents sometimes trade goods and financial assets as in Walrasian theory (in well-organized markets, at market-clearing prices), and sometimes as in search theory (in a decentralized manner, with the terms of trade determined by bargaining). Decentralized trade combined with an exchange motive generates the need for a medium of exchange. The equilibrium price and rate of return of a financial asset are partly determined by the asset's usefulness to facilitate exchange. When an asset is held partly for its exchange value, its price will be higher, and its measured rate of return - which takes into account only the stream of consumption goods that the asset represents - will be lower than they would be if the asset were not used as a medium of exchange.

In Section 2, I consider an economy with two assets: an equity share and a one-period government-issued risk-free real bill. In the basic setup, assets differ only in their payoffs, and agents are free to choose which assets to use as means of payment in decentralized trades. In this case, the theory unambiguously predicts that someone testing an agent's Euler equation for the risk-free bill using its measured rate of return would find that, at the margin, this agent can gain from transferring consumption from the future to the present. That is, there would appear to be a risk-free rate puzzle.

I also analyze versions of the economy in which institutional or legal restrictions give bonds an advantage over equity as a medium of exchange. In this case, it is possible to show that there are degrees of these restrictions for which someone testing an agent's Euler equation for the measured excess returns would find that, at the margin, the agent can gain from disinvesting in bills and investing in equity: there would appear to be an equity-premium puzzle. For this class

of economies, the risk-free rate would still seem too low to an outside observer. In fact, the risk- 
free rate will be even lower than it would be in the absence of institutional or legal restrictions. Without these restrictions, the theory may still be consistent with an equity-premium puzzle, depending on parameter values. These issues are analyzed in Section 3.

In Section 4, I calibrate the model economies and study the extent to which they are able to generate average equity returns and risk-free rates that are in line with U.S. data. Since the class of model economies I consider nests the one studied by Mehra and Prescott (1985), I can quantify the degree to which the liquidity mechanism considered here can help explain the anomalies they identified. Mehra and Prescott's test of their theory essentially consisted of experimenting with different values of the curvature of the agent's utility function (call it $\sigma$ ) to find the values for which the average risk-free rate and equity premium in the model matched those in the U.S. economy. I carry out a similar exercise.

First, I consider the economy with no legal or institutional differences between equity shares and bills, and assess the ability of the model to produce risk-free rates and equity premia that match the data for values of $\sigma$ ranging from 1 to 10. I find that for values of $\sigma$ up to 7 , the liquidity mechanism is inactive, and the equilibrium looks just like the one in Mehra-Prescott. For values of $\sigma$ equal to or greater than 8, equity shares and bills are valuable in decentralized exchange at the margin. This lowers the return on equity and the risk-free rate from what they would be in the Mehra-Prescott economy and brings them closer to the data. However, relative to the data, for this range of $\sigma$ the equity return is a bit too low and the risk-free rate a bit too high, so the average equity premium is still too low.

I also consider the specification with legal or institutional restrictions, in which equity shares cannot be used as means of payment in a fraction $\theta$ of decentralized exchanges. I report how asset returns vary with $\theta$ in general and formulate the following question: For a given value of $\sigma$, how large does $\theta$ (the relative "illiquidity" of equity) have to be for the model to generate an average risk-free rate of $1 \%$ and an average equity premium that matches the long-term average for the U.S. economy? The answer is, quite small. Section 4.3 offers a way to assess the absolute size of the implied $\theta$ by relating it to relative trade volumes of equity and bonds. 
In Section 5, I discuss how the liquidity mechanism operates in this model, and what it adds to the Mehra-Prescott model, by contrasting how the mean and standard deviation of their respective stochastic discount factors fare against the bounds of Hansen and Jagannathan (1991). In this section, I also use the model to decompose the equity premium into two components: a pure risk premium for bearing nondiversifiable aggregate consumption risk, and an illiquidity premium related to bonds being easier to trade away if a decentralized trade opportunity arises.

By now a vast literature seeks to solve the puzzle identified by Mehra and Prescott (1985). As they framed it, the puzzle is the observation that the restrictions that a particular class of general equilibrium models places upon average returns of equity and Treasury bills are violated by U.S. data. This particular class of models has: (i) agents who maximize the expected discounted value of a stream of utilities generated by a power utility function; ( $i i)$ "frictionless" trading (e.g., no brokerage fees or other trading or transaction costs); and (iii) complete asset markets (agents can write insurance contracts against any contingency).

The literature spurred by the work of Mehra and Prescott can be classified depending on which of these ingredients it alters. ${ }^{1}$ Looked at from this angle, this paper relaxes ( $\left.i i\right)$ and (iii). There are trading frictions in the sense that agents sometimes trade bilaterally instead of in a Walrasian marketplace. Markets are incomplete in that agents cannot make binding commitments, and trading histories are private in a way that precludes any borrowing and lending between people. Therefore, all trade - both in the centralized and decentralized markets - must be quid pro quo. In terms of this broad taxonomy, this paper is related to Aiyagari and Gertler (1991). They consider an economy with equity and government bonds in which agents face idiosyncratic shocks, and markets are incomplete in a way that individual agents must self-insure. In their model, agents hold assets not only for the stream of dividends they yield but also as a vehicle of self-insurance. This alone can help to lower the risk-free rate. The basic logic of this mechanism is similar to the one I am emphasizing, except that here, the additional motive for holding assets is their role in transactions rather than self-insurance

\footnotetext{
${ }^{1}$ I will not attempt to list all the relevant work in the area. See Mehra and Prescott (2003) for references.
} 
considerations. $^{2}$ At a conceptual level, the paper also shares the basic premise of Bansal and Coleman (1996) and Kiyotaki and Moore (2005), namely, that an asset's role in facilitating some form of exchange will manifest itself in the equity premium and as a risk-free rate puzzle.

This paper is also closely related to the literature that provides micro foundations for monetary economics based on search theory, as pioneered by Kiyotaki and Wright (1989). This approach has proven useful for understanding the nature of monetary exchange by making explicit the frictions - e.g., the configuration of meetings, specialization patterns, information structure, and so on - that make monetary exchange an equilibrium. Put differently, this approach has proven useful in pricing the most elusive among financial assets: fiat money, an asset that is a formal claim to nothing yet sells at a positive price. Somehow, this literature and the mainstream asset-pricing literature have managed to stay disconnected. ${ }^{3}$ Recently, Duffie, Gârleanu, and Pedersen (2005a,b), Vayanos and Wang (2005), Vayanos and Weill (2005), and Weill (2005a,b) have begun to build some interesting connections between both fields. They model asset trading as a decentralized exchange process that resembles the original vintages of the equilibrium search models of Diamond (1982) and Kiyotaki and Wright (1989). This paper also bridges these fields, in the precise sense that the model can be viewed as a blend of Lagos and Wright (2005) — a recent vintage of the search-based model of exchange - and Lucas (1978). ${ }^{4}$

\footnotetext{
${ }^{2}$ To widen the spread between the risk-free rate and the return on equity, Aiyagari and Gertler (1991) introduce differential (proportional) trading costs across equity and bonds. If transaction costs on bonds are lower than on equity, then in equilibrium equity must pay a premium, which they refer to as a "transactions/liquidity premium." They also emphasize the model implications for the volumes of trade for bonds and equity as a way of assessing the plausibility of the magnitudes of the trading costs that they feed into the model. Heaton and Lucas (1996) analyze an economy similar to the one in Aiyagari and Gertler, but they allow for aggregate uncertainty. Other papers that consider various combinations of transaction costs and short-sale constraints include Telmer (1993), Lucas (1994), He and Modest (1995), and Luttmer (1996). See Heaton and Lucas (1995) for a survey.

${ }^{3}$ Mehra and Prescott (1985) were the first to point out the similarities between the equity premium puzzle and the rate-of-return-dominance puzzle that pervades the pure theory of money. Kocherlakota (1996) picked up on this theme in his concluding section. I will return to it in mine.

${ }^{4}$ Contemporaneously, Ravikumar and Shao (2005) are working on a related model that instead combines features of Lucas (1978) with features of Lagos and Wright (2005) and Shi (1997). Our papers clearly share much ground: both investigate how the role that an asset plays in the exchange process affects its equilibrium price. As for differences - aside from several in terms of modeling - their agents trade a single asset (equity), as the model is designed to address the excess volatility puzzle rather than the equity premium puzzle and the risk-free rate puzzle. So all in all, our work is decidedly complementary.
} 


\section{The model}

There is a $[0,1]$ continuum of agents, time is discrete and the horizon is infinite. Each period is divided into two subperiods where different activities take place. There are three nonstorable and perfectly divisible consumption goods at each date: general goods, and two types of fruit, which I will refer to as apples and coconuts. (By "nonstorable" I mean that the goods cannot be carried from one subperiod to the next.) Let $Z_{t}$ be an aggregate productivity index, and assume that $Z_{t+1}=x_{t+1} Z_{t}$, where $x_{t+1} \in\left\{\gamma_{1}, \ldots, \gamma_{n}\right\}$ and $\operatorname{Pr}\left(x_{t+1}=\gamma_{j} \mid x_{t}=\gamma_{i}\right)=\mu_{i j}$. (The conditional expectation, $E_{t}$, used below, is defined with respect to this transition probability. Throughout, I will assume that the Markov chain is ergodic.) The realization of $x_{t}$ becomes known at the beginning of period $t$.

The only durable commodity in the economy is a set of "Lucas trees." The number of trees is fixed and equal to the number of agents. Trees yield a quantity $d_{t}=Z_{t}$ of applesthe "dividend" - in the second subperiod of every period. Production of apples is entirely exogenous: no resources are utilized, and it is not possible to affect the output at any time. In the second subperiod, every agent is also endowed with $\bar{n}$ units of time and has access to a linear production technology that allows him to transform each unit of time into $Z_{t}$ units of general goods. ${ }^{5}$

In the first subperiod, half of the agents are endowed with $(1+\epsilon) \kappa Z_{t}$ coconuts, and the remaining half with $(1-\epsilon) \kappa Z_{t}$ coconuts, where $\epsilon \in[0,1]$, and $\kappa>0$. Thus, the total endowment of coconuts in period $t$ equals $\kappa Z_{t}$. The agents who receive the high endowment are selected at random from the population at the beginning of each period, so from the perspective of an individual agent, the endowment process is independent across periods. ${ }^{6}$ Note that there is a precise sense in which $Z_{t}$ indexes the economy-wide state of technology: when $Z_{t}$ is relatively

\footnotetext{
${ }^{5}$ This formulation is in some sense the most straightforward integration of the asset-pricing model of Lucas (1978) with the model of exchange in Lagos and Wright (2005). In a different context, I have considered other formulations, for example, one where instead of being consumption goods, apples are a necessary input - together with labor - in the production of the general consumption good. (See the appendix to Lagos (2006) for details.)

${ }^{6}$ It would not be difficult to allow the individual endowment process to be correlated over time. Reed and Waller (2005) use a similar formulation in their study of monetary policy and risk sharing.
} 
high, the Lucas trees yield more apples, the technology that transforms labor into general goods is more efficient, and there is a larger aggregate endowment of coconuts.

Let $u(Q)$ be the utility from consuming $Q$ coconuts in the first subperiod, and let $U(c)$ and $v(y)$ represent the utilities from consuming $c$ apples and $y$ general goods in the second subperiod. Each agent $i$ wishes to maximize

$$
E_{0}\left\{\sum_{t=0}^{\infty} \beta^{t}\left[u\left(Q_{t}^{i}\right)+U\left(c_{t}^{i}\right)+v\left(y_{t}^{i}\right)-A_{t} h_{t}^{i}\right]\right\},
$$

where $\beta \in(0,1), Q_{t}^{i}$ is agent $i$ 's consumption of coconuts in the first subperiod, $c_{t}^{i}$ and $y_{t}^{i}$ are his consumption of apples and general goods, and $h_{t}^{i}$ is his labor supply in the second subperiod. The marginal disutility from working is $A_{t}>0 .^{7}$ Assume $u^{\prime}>0, v^{\prime}>0, U^{\prime}>0, u^{\prime \prime}<0$, $v^{\prime \prime} \leq 0$, and $U^{\prime \prime}<0$. It is also convenient to have $u^{\prime}(0)=v^{\prime}(0)=U^{\prime}(0)=+\infty$.

In the second subperiod, agents trade apples, labor, general goods, and financial assets in a centralized (Walrasian) market. In the first subperiod, agents trade coconuts and financial assets in a decentralized market where trade is bilateral (each meeting is a random draw from the set of pairwise meetings), and the terms of trade are determined by bargaining. Let $\hat{\alpha}$ denote the probability of a meeting. Bilateral trade, together with the fact that some agents receive a high endowment and others a low endowment of coconuts, gives rise to a doublecoincidence-of-wants problem in the first subperiod. There are two relevant types of meetings: $\hat{\alpha} / 2$ of them involve an agent with a high endowment and an agent with a low endowment (naturally, in these "single-coincidence meetings," the agent with the low endowment will be the buyer and the other the seller); and $\hat{\alpha} / 2$ of the meetings are "no-coincidence meetings" that involve either two agents with high endowment or two agents with low endowment. Agents cannot make binding commitments, and trading histories are private in a way that precludes any borrowing and lending between people, so all trade - both in the centralized and decentralized markets - must be quid pro quo.

\footnotetext{
${ }^{7}$ The $t$ subscript on $A_{t}$ is meant to allow for specifications where the disutility of work depends on the aggregate technology index, $Z_{t}$. The usefulness of such specifications has to do with balanced growth considerations, as discussed below.
} 
Each tree has outstanding one durable and perfectly divisible equity share that represents the bearer's ownership of a tree and confers him the right to collect the apple dividends. There is a second perfectly divisible asset, a one-period risk-free government-issued real bill. (Each of these "bonds" pays off an apple at maturity.) Let $B_{t}$ denote the stock of bonds that are outstanding in period $t$, to be redeemed before the centralized trading session of period $t$. (The government sells $B_{t+1}$ in the centralized market at the end of period $t$.) What I call the "government" is essentially summarized by the budget constraint $B_{t}=\phi_{t}^{b} B_{t+1}+\tau_{t}$, where $\phi_{t}^{b}$ is the price of a bond and $\tau_{t}$ a lump-sum tax levied on all agents during the centralized trading session, both expressed in terms of apples. The focus here will not be on how the government should select the path $\left\{B_{t}, \tau_{t}\right\}$, but rather on characterizing the equilibrium, and in particular asset prices and returns, given such a path. All assets are perfectly recognizable, cannot be forged, and can be traded among agents in the centralized and decentralized markets. At $t=0$ each agent is endowed with $a_{0}^{s}$ equity shares and $a_{0}^{b}$ units of the bond.

In the first subperiod, endowment inequality gives agents a motive for trade. In addition, the combination of bilateral meetings and quid pro quo trade generates the need for an asset to act as a medium of exchange. But which of the two assets will play this role - bonds or equity shares? From a theoretical standpoint, one could adhere to Wallace's dictum (e.g., Wallace 1998) and argue that in laying down the environment, one should specify assets only by their physical properties, not by the role they play. That is, given the physical characteristics of the various assets (their payoffs or other inherent properties such as their divisibility, recognizability, etc.), the theory ought to determine their roles in exchange endogenously — as part of the equilibrium. I consider a baseline version of the model that adheres to the dictum. In this version, assets differ only in their payoffs, and there are no exogenous liquidity differences between bonds and equity shares: the agents in the model are free to choose which asset or combination of assets to use in exchange. But since the focus of the paper is on a primarily applied question, namely, the extent to which an asset's usefulness in exchange affects its measured return relative to other assets, I also consider a more general formulation that allows for the possibility that 
bonds and equity shares may have different exogenous liquidity properties. Specifically, I will suppose that an agent can find himself in two types of meetings in the decentralized market: with probability $\theta_{2}$, he is in a meeting where he can use any of the two assets for payment, whereas with probability $\theta_{1}=1-\theta_{2}$, he is able to use only bonds. ${ }^{8}$ Thus, $\theta_{1} \in[0,1]$ indexes the degree of "illiquidity" of equity shares. (The subscript refers to the number of assets that can be used for payment in that particular type of meeting.) The formulation that satisfies Wallace's dictum corresponds to the special case of $\theta_{1}=0$. In the remainder of the section, I describe the problems that agents face in both the decentralized and centralized markets, characterize the terms of trade that will prevail in bilateral meetings, and introduce the notion of equilibrium.

I begin by formulating the Bellman equations that summarize the individual agents' optimization problem. Let $\mathbf{a}_{t} \in \mathbb{R}_{+}^{2}$ denote an agent's portfolio. Since agents can hold two assets, $\mathbf{a}_{t}=\left(a_{t}^{b}, a_{t}^{s}\right)$, where $a_{t}^{b}$ and $a_{t}^{s}$ denote the holdings of bonds and shares, respectively. Let $W\left(\mathbf{a}_{t+1}, \mathbf{s}_{t+1}\right)$ be the value of an agent who enters the centralized market holding portfolio $\mathbf{a}_{t+1}$ in a period when the aggregate state of the economy is $\mathbf{s}_{t+1}=\left(d_{t+1}, x_{t+1}, B_{t+1}\right)$, i.e., in a period when the realization of the endowment process is $d_{t+1}=x_{t+1} d_{t}$ and there are $B_{t+1}$ bonds in circulation. Similarly, let $V_{j}\left(\mathbf{a}_{t+1}, \mathbf{s}_{t+1}\right)$ be the value of search for an agent who enters the

\footnotetext{
${ }^{8}$ To interpret the specific modeling choice, one can follow Aiyagari and Wallace (1997) and relate $\theta_{1}$ to a government transaction policy carried out by a small mass of government agents. This has become the standard way of introducing legal or other institutional restrictions into environments with decentralized exchange. See, for example, Aiyagari, Wallace, and Wright (1996) or Li and Wright (1998). Also, more recently, Shi (2005) has used a similar formulation in a model with fiat money and nominal bonds to study the effects of open market operations. He finds that even an arbitrarily small probability that matured nominal bonds will not be accepted in decentralized exchange is enough for fiat money to drive them out of circulation. Regarding my use of this device, I would like to emphasize that the spirit of this exercise is primarily positive. That is, here I want to explore the implications of (small) liquidity differences for the behavior of asset prices in general, and for the equity premium and risk-free rate puzzles in particular. In fact, given the nature of the findings, I will argue that understanding the deeper reasons for these differences in the liquidity of these assets - by which I mean the differences in the likelihood they can serve as means of payment in decentralized exchange - is a necessary next step. In terms of relating these theoretical institutional or legal restrictions to actual features of "real world" trades, consider the following readily verifiable fact. An investor who places an order to sell shares of an S\&P 500 firm on a given day $T$ will typically have to wait until $T+2$ for settlement, whereas the settlement for the sale of 90-day U.S. Treasury bills will usually take place at $T+1$. These types of considerations seem to suggest that bonds are a more readily available source of funds for agents who must act quickly on some purchase opportunity, which is at least broadly in line with the trade-offs at work in the theoretical formulation laid out here.
} 
decentralized market holding portfolio $\mathbf{a}_{t+1}$ in a period when the aggregate state is $\mathbf{s}_{t+1}$. The subindex $j \in\{h, l\}$ indicates whether the agent has a high $(j=h)$ or a low $(j=l)$ endowment of coconuts.

Since there are, in general, two types of bilateral meetings - unrestricted matches where the buyer can use both assets as means of payment, and restricted matches where he can use only bonds - there are two sets of terms of trade in the decentralized market. The terms of trade in a meeting of type $i$ between a buyer with portfolio $\mathbf{a}_{t}=\left(a_{t}^{b}, a_{t}^{s}\right)$ and a seller with portfolio $\tilde{\mathbf{a}}_{t}=\left(\tilde{a}_{t}^{b}, \tilde{a}_{t}^{s}\right)$ are $\left[q^{i}\left(\mathbf{a}_{t}, \tilde{\mathbf{a}}_{t}\right), \mathbf{p}^{i}\left(\mathbf{a}_{t}, \tilde{\mathbf{a}}_{t}\right)\right]$, where $\mathbf{p}^{i}\left(\mathbf{a}_{t}, \tilde{\mathbf{a}}_{t}\right) \in \mathbb{R}_{+}^{2}$ is the portfolio that the buyer hands over to the seller in exchange for $q^{i}\left(\mathbf{a}_{t}, \tilde{\mathbf{a}}_{t}\right) \in \mathbb{R}_{+}$coconuts. ${ }^{9}$ Then,

$$
\begin{aligned}
V_{h}(\mathbf{a}, \mathbf{s})= & \frac{\hat{\alpha}}{2} \sum_{i=1,2} \theta_{i} \int\left\{u\left[(1+\epsilon) \kappa d-q^{i}(\tilde{\mathbf{a}}, \mathbf{a})\right]+W\left[\mathbf{a}+\mathbf{p}^{i}(\tilde{\mathbf{a}}, \mathbf{a}), \mathbf{s}\right]\right\} d \mathbf{G}(\tilde{\mathbf{a}}) \\
& +\left(1-\frac{\hat{\alpha}}{2}\right)\{u[(1+\epsilon) \kappa d]+W(\mathbf{a}, \mathbf{s})\},
\end{aligned}
$$

where $\mathbf{G}$ denotes the distribution of portfolios across agents. Similarly,

$$
\begin{aligned}
V_{l}(\mathbf{a}, \mathbf{s})= & \frac{\hat{\alpha}}{2} \sum_{i=1,2} \theta_{i} \int\left\{u\left[(1-\epsilon) \kappa d+q^{i}(\mathbf{a}, \tilde{\mathbf{a}})\right]+W\left[\mathbf{a}-\mathbf{p}^{i}(\mathbf{a}, \tilde{\mathbf{a}}), \mathbf{s}\right]\right\} d \mathbf{G}(\tilde{\mathbf{a}}) \\
& +\left(1-\frac{\hat{\alpha}}{2}\right)\{u[(1-\epsilon) \kappa d]+W(\mathbf{a}, \mathbf{s})\} .
\end{aligned}
$$

The expected value of an agent entering the decentralized market with portfolio $a$ in a period when the aggregate state is $\mathbf{s}$, before knowing his endowment of coconuts, is $V(\mathbf{a}, \mathbf{s})=$ $\frac{1}{2}\left[V_{l}(\mathbf{a}, \mathbf{s})+V_{h}(\mathbf{a}, \mathbf{s})\right] ;$ i.e.,

$$
\begin{aligned}
V(\mathbf{a}, \mathbf{s})= & \alpha \sum_{i=1,2} \theta_{i} \int\left\{u\left[(1-\epsilon) \kappa d+q^{i}(\mathbf{a}, \tilde{\mathbf{a}})\right]+W\left[\mathbf{a}-\mathbf{p}^{i}(\mathbf{a}, \tilde{\mathbf{a}}), \mathbf{s}\right]\right\} d \mathbf{G}(\tilde{\mathbf{a}}) \\
& +\alpha \sum_{i=1,2} \theta_{i} \int\left\{u\left[(1+\epsilon) \kappa d-q^{i}(\tilde{\mathbf{a}}, \mathbf{a})\right]+W\left[\mathbf{a}+\mathbf{p}^{i}(\tilde{\mathbf{a}}, \mathbf{a}), \mathbf{s}\right]\right\} d \mathbf{G}(\tilde{\mathbf{a}}) \\
& +(1-2 \alpha)[\bar{u}(d)+W(\mathbf{a}, \mathbf{s})],
\end{aligned}
$$

\footnotetext{
${ }^{9}$ In an unrestricted match $\left(i=2\right.$, with probability $\left.\theta_{2}\right), \mathbf{p}^{2}\left(\mathbf{a}_{t}, \tilde{\mathbf{a}}_{t}\right)=\left[p^{b}\left(\mathbf{a}_{t}, \tilde{\mathbf{a}}_{t}\right), p^{s}\left(\mathbf{a}_{t}, \tilde{\mathbf{a}}_{t}\right)\right]$, where $p^{b}\left(\mathbf{a}_{t}, \tilde{\mathbf{a}}_{t}\right)$ denotes the quantity of bonds and $p^{s}\left(\mathbf{a}_{t}, \tilde{\mathbf{a}}_{t}\right)$ the quantity of shares that the buyer hands over to the seller. In restricted matches $\left(i=1\right.$, with probability $\left.\theta_{1}\right), \mathbf{p}^{1}\left(\mathbf{a}_{t}, \tilde{\mathbf{a}}_{t}\right)=\left[p^{b}\left(\mathbf{a}_{t}, \tilde{\mathbf{a}}_{t}\right), 0\right]$.
} 
where $\alpha \equiv \hat{\alpha} / 4$, and $\bar{u}(d) \equiv \frac{1}{2}\{u[(1+\epsilon) \kappa d]+u[(1-\epsilon) \kappa d]\}{ }^{10}$

The value function of an agent who enters the centralized market with portfolio $\mathbf{a}_{t}$ in $\mathrm{a}$ period when the aggregate state is $\mathbf{s}_{t}$ satisfies

$$
\begin{gathered}
W\left(\mathbf{a}_{t}, \mathbf{s}_{t}\right)=\max _{c_{t}, y_{t}, n_{t}, h_{t}, \mathbf{a}_{t+1}}\left\{U\left(c_{t}\right)+v\left(y_{t}\right)-A_{t} h_{t}+\beta E_{t} V\left(\mathbf{a}_{t+1}, \mathbf{s}_{t+1}\right)\right\} \\
\text { s.t. } c_{t}+w_{t} n_{t}+\phi_{t} \mathbf{a}_{t+1}=\left(\phi_{t}^{s}+d_{t}\right) a_{t}^{s}+a_{t}^{b}+w_{t} h_{t}-\tau_{t} \\
0 \leq c_{t}, 0 \leq n_{t}, 0 \leq h_{t} \leq \bar{n}, 0 \leq \mathbf{a}_{t+1}, y_{t}=Z_{t} n_{t} .
\end{gathered}
$$

The agent chooses consumption of apples $\left(c_{t}\right)$ and of the general good $\left(y_{t}\right)$, how many hours of work to demand $\left(n_{t}\right)$ and supply $\left(h_{t}\right)$, and an end-of-period portfolio $\left(\mathbf{a}_{t+1}\right)$. Dividends are paid to the bearer of the equity share after decentralized trade, but before the time- $t$ centralized trading session. Apples are used as numeraire, $w_{t}$ is the real wage, $\phi_{t}^{b}$ the real price of a bond, $\phi_{t}^{s}$ the real price of a share (ex-dividend), with $\phi_{t}=\left(\phi_{t}^{b}, \phi_{t}^{s}\right)$, and $\tau_{t}$ is a lump-sum tax also expressed in terms of apples. Let $\lambda_{t}^{b}=\frac{A_{t}}{w_{t}}, \lambda_{t}^{s}=\left(\phi_{t}^{s}+d_{t}\right) \lambda_{t}^{b}$, and $\boldsymbol{\lambda}_{t}=\left(\lambda_{t}^{b}, \lambda_{t}^{s}\right)$. Substituting the budget constraint and $y_{t}=Z_{t} n_{t}$ into the objective, the problem becomes

$$
\begin{aligned}
W\left(\mathbf{a}_{t}, \mathbf{s}_{t}\right)= & \boldsymbol{\lambda}_{t} \mathbf{a}_{t}-\lambda_{t}^{b} \tau_{t}+\max _{c_{t}, n_{t}}\left[U\left(c_{t}\right)+v\left(Z_{t} n_{t}\right)-\frac{A_{t}}{w_{t}}\left(c_{t}+w_{t} n_{t}\right)\right] \\
& +\max _{\mathbf{a}_{t+1}}\left[-\frac{A_{t}}{w_{t}} \boldsymbol{\phi}_{t} \mathbf{a}_{t+1}+\beta E V\left(\mathbf{a}_{t+1}, \mathbf{s}_{t+1}\right)\right],
\end{aligned}
$$

with $h_{t}=\frac{1}{w_{t}}\left[c_{t}+w_{t} n_{t}+\phi_{t} \mathbf{a}_{t+1}-\left(\phi_{t}^{s}+d_{t}\right) a_{t}^{s}-a_{t}^{b}+\tau_{t}\right]$, and subject to $0 \leq c_{t}, 0 \leq n_{t}$, $0 \leq \mathbf{a}_{t+1}$, and $0 \leq h_{t} \leq \bar{n}^{11}$

Consider a meeting of type $i$ in the decentralized market between a buyer holding portfolio $\mathbf{a}$ and a seller holding $\tilde{\mathbf{a}}$. The terms of trade $\left[q^{i}(\mathbf{a}, \tilde{\mathbf{a}}), \mathbf{p}^{i}(\mathbf{a}, \tilde{\mathbf{a}})\right]$, for $i=1,2$, are determined by

\footnotetext{
${ }^{10}$ Note that this expression for the value of search is essentially the same as the one in Lagos (2006), except that here, the utility gain of a buyer is $u\left[(1-\epsilon) \kappa d+q^{i}(\mathbf{a}, \tilde{\mathbf{a}})\right]-u[(1-\epsilon) \kappa d]$ instead of $u\left[q^{i}(\mathbf{a}, \tilde{\mathbf{a}})\right]$, the utility cost of a seller is $u[(1+\epsilon) \kappa d]-u\left[(1+\epsilon) \kappa d-q^{i}(\tilde{\mathbf{a}}, \mathbf{a})\right]$ instead of $e\left[q^{i}(\tilde{\mathbf{a}}, \mathbf{a})\right]$, and agents without a trade opportunity consume $(1+\epsilon) \kappa d$ or $(1-\epsilon) \kappa d$, each with probability a half, instead of 0 with certainty.

${ }^{11}$ Note that the choice of next period portfolio, $\mathbf{a}_{t+1}$, is independent of the current portfolio, $\mathbf{a}_{t}$. Thus, as in Lagos and Wright (2005), a unique solution to the portfolio maximization problem implies that the distribution of portfolios, G, must be degenerate (and equal to the mean holding) in the equilibrium, as long as $h_{t}$ is interior. In the appendix (Section A.2), I derive parametric conditions such that this is indeed the case along the equilibrium path. The nonnegativity constraints on $c_{t}$ and $n_{t}$ will not bind because $U^{\prime}(0)=v^{\prime}(0)=+\infty$. Also, bonds and shares will be valued in equilibrium, and someone has to hold them. Thus, $0 \leq \mathbf{a}_{t+1}$ will not bind either.
} 
Nash bargaining where the buyer has all the bargaining power. ${ }^{12}$ Thus, for $i=1,2,\left(q^{i}, \mathbf{p}^{i}\right)$ solves

$$
\begin{aligned}
& \max _{q^{i}, \mathbf{p}^{i}}\left\{u\left[(1-\epsilon) \kappa d+q^{i}\right]+W\left(\mathbf{a}-\mathbf{p}^{i}, \mathbf{s}\right)-u[(1-\epsilon) \kappa d]-W(\mathbf{a}, \mathbf{s})\right\} \\
& \text { s.t. } u\left[(1+\epsilon) \kappa d-q^{i}\right]+W\left(\tilde{\mathbf{a}}+\mathbf{p}^{i}, \mathbf{s}\right)-u[(1+\epsilon) \kappa d]-W(\tilde{\mathbf{a}}, \mathbf{s}) \geq 0,
\end{aligned}
$$

and subject to $\mathbf{p}^{1}=\left(p^{b}, 0\right)$ and $p^{b} \leq a^{b}$ if $i=1$ (matches where only bonds can be used as means of payment), or subject to $\mathbf{p}^{2}=\left(p^{b}, p^{s}\right) \leq \mathbf{a}$ if $i=2$ (matches where both bonds and shares can be used for payment). The agent cannot spend more than all the assets he owns in an unrestricted match, nor more than all the bonds he owns in a restricted match. In what follows, I will refer to these trading constraints as "liquidity constraints." Define the function $q(z ; y): \mathbb{R}_{+}^{2} \rightarrow \mathbb{R}_{+}$as

$$
q(z ; y)= \begin{cases}\epsilon y & \text { if } z \geq u[(1+\epsilon) y]-u(y) \\ \hat{q}(z ; y) & \text { if } z<u[(1+\epsilon) y]-u(y)\end{cases}
$$

where $\hat{q}(z ; y)$ denotes the $q$ that solves $u[(1+\epsilon) y]-u[(1+\epsilon) y-q]=z$. Then, for $i=1,2$, the bargaining solution in period $t$ has $q^{i}\left(\mathbf{a}_{t}, \tilde{\mathbf{a}}_{t}\right)=q\left(\boldsymbol{\lambda}_{t}^{i} \mathbf{a}_{t} ; \kappa d_{t}\right)$, where $\boldsymbol{\lambda}_{t}^{1} \equiv\left(\lambda_{t}^{b}, 0\right)$ and $\boldsymbol{\lambda}_{t}^{2}=\boldsymbol{\lambda}_{t}$.

Given the dividend process $\left\{d_{t}\right\}_{t=0}^{\infty}$ and a path $\left\{B_{t}, \tau_{t}\right\}_{t=0}^{\infty}$, an equilibrium is an allocation $\left\{c_{t}, y_{t}, n_{t}, h_{t}, \mathbf{a}_{t+1}\right\}_{t=0}^{\infty}$, together with a set of prices $\left\{w_{t}, \phi_{t}\right\}_{t=0}^{\infty}$ and bilateral terms of trade $\left\{\left(q_{t}^{i}\right)_{i=1,2}\right\}_{t=0}^{\infty}$, such that: $(i)$ the individual choices $\left\{c_{t}, y_{t}, n_{t}, h_{t}, \mathbf{a}_{t+1}\right\}_{t=0}^{\infty}$ solve the agent's problem in the centralized market, given prices; $(i i)$ the terms of trade in bilateral meetings are determined by Nash bargaining, i.e., $q_{t}^{1}=q\left(\lambda_{t}^{b} a_{t}^{b} ; \kappa d_{t}\right)$ and $q_{t}^{2}=q\left(\boldsymbol{\lambda}_{t} \mathbf{a}_{t} ; \kappa d_{t}\right)$; and $(i i i)$ prices are such that the centralized market clears, i.e., $c_{t}=d_{t}, a_{t+1}^{b}=B_{t+1}, a_{t+1}^{s}=1$, and the government budget constraint, $B_{t}=\phi_{t}^{b} B_{t+1}+\tau_{t}$, is satisfied.

\footnotetext{
${ }^{12}$ In the appendix (Section A.5), I study the general case in which the buyer has bargaining power $\eta \in[0,1]$, both analytically and quantitatively.
} 


\section{The equity premium and the risk-free rate puzzles}

In equilibrium, asset prices $\left\{\phi_{t}\right\}_{t=0}^{\infty}$ satisfy

$$
\begin{aligned}
U^{\prime}\left(d_{t}\right) \phi_{t}^{s} & =\beta E_{t} U^{\prime}\left(d_{t+1}\right) L_{t+1}^{s}\left[\phi_{t+1}^{s}+d_{t+1}\right] \\
U^{\prime}\left(d_{t}\right) \phi_{t}^{b} & =\beta E_{t} U^{\prime}\left(d_{t+1}\right) L_{t+1}^{b}
\end{aligned}
$$

with

$$
\begin{aligned}
& L_{t+1}^{s}=1+\alpha(1-\theta)\left\{\frac{u^{\prime}\left[(1-\epsilon) \kappa d_{t+1}+q_{t+1}^{2}\right]}{u^{\prime}\left[(1+\epsilon) \kappa d_{t+1}-q_{t+1}^{2}\right]}-1\right\} \\
& L_{t+1}^{b}=L_{t+1}^{s}+\alpha \theta\left\{\frac{u^{\prime}\left[(1-\epsilon) \kappa d_{t+1}+q_{t+1}^{1}\right]}{u^{\prime}\left[(1+\epsilon) \kappa d_{t+1}-q_{t+1}^{1}\right]}-1\right\}
\end{aligned}
$$

where $q_{t+1}^{i}=q\left(\boldsymbol{\lambda}_{t+1}^{i} \mathbf{a}_{t+1}, \kappa d_{t+1}\right)$ for $i=1,2$, with $\lambda_{t+1}^{b}=U^{\prime}\left(d_{t+1}\right), \lambda_{t+1}^{s}=\left(\phi_{t+1}^{s}+d_{t+1}\right) \lambda_{t+1}^{b}$, $\mathbf{a}_{t+1}=\left(B_{t+1}, 1\right)$, and $\theta \equiv \theta_{1} \cdot{ }^{13}$ The factors $L_{t+1}^{s}$ and $L_{t+1}^{b}$ have a natural interpretation as the liquidity returns from holding shares and bonds, respectively. Note that $L_{t+1}^{i} \geq 1$ for $i=b, s$, with strict inequality if the trading constraint $\mathbf{p}^{i} \leq \mathbf{a}$ in the decentralized market is binding.

To illustrate, let $\theta=0$, i.e., suppose that all matches are unrestricted. Then, $L_{t+1}^{s}=L_{t+1}^{b}=$ $L_{t+1}$ in all states. In a state $\mathbf{s}_{t+1}$ in which $\boldsymbol{\lambda}_{t+1} \mathbf{a}_{t+1}<u\left[(1+\epsilon) \kappa d_{t+1}\right]-u\left(\kappa d_{t+1}\right)$, buyers do not have enough real value of assets to buy $\epsilon \kappa d_{t+1}$, so $L_{t+1}>1$. In fact, $L_{t+1}-1$ is the slope of the gains from trade that accrue to the buyer, or equivalently, $L_{t+1}$ is the expected marginal benefit that the buyer gets from bringing the value of an additional apple's worth of liquidity into the search market, i.e., $L_{t+1}=(1-\alpha)+\alpha \frac{\partial q_{t+1}}{\partial\left(\boldsymbol{\lambda}_{t+1} \mathbf{a}_{t+1}\right)} u^{\prime}\left[(1-\epsilon) \kappa d_{t+1}+q_{t+1}\right]$. With probability $(1-\alpha)$ the agent has no use for the asset in the decentralized market, and hence he gets no additional return from holding it. But with probability $\alpha$ he is a buyer in the search market, and the additional value of assets he brings into the meeting allows him to obtain $\frac{\partial q_{t+1}}{\partial\left(\boldsymbol{\lambda}_{t+1} \mathbf{a}_{t+1}\right)}$ additional coconuts from the seller, which at the margin, he (the buyer) values at $u^{\prime}\left[(1-\epsilon) \kappa d_{t+1}+q_{t+1}\right]$. Since $\frac{\partial q_{t+1}}{\partial\left(\boldsymbol{\lambda}_{t+1} \mathbf{a}_{t+1}\right)}=\frac{1}{u^{\prime}\left[(1+\epsilon) \kappa d_{t+1}-q_{t+1}\right]}$, we have $L_{t+1}=$ $(1-\alpha)+\alpha \frac{u^{\prime}\left[(1-\epsilon) \kappa d_{t+1}+q_{t+1}\right]}{u^{\prime}\left[(1+\epsilon) \kappa d_{t+1}-q_{t+1}\right]}$, which corresponds to $(4)$ with $\theta=0$. In a state $\mathbf{s}_{t+1}$ in which

\footnotetext{
${ }^{13}$ The Euler equations (2) and (3) are derived in the appendix (Section A.1).
} 
the agent has enough real value of assets to afford the quantity of coconuts that maximizes the gains from trade, namely, $\epsilon \kappa d_{t+1}$, he will buy $q_{t+1}=\epsilon \kappa d_{t+1}$ coconuts, and therefore, $L_{t+1}=1$.

In general, for $\theta \in(0,1]$, (4) and (5) imply $1 \leq L_{t+1}^{s} \leq L_{t+1}^{b}$. The second inequality is due to the fact that bonds provide liquidity (they can be used to relax the trading constraint at the margin) whenever shares do, but in addition, they can also be used as means of payment in the fraction $\theta$ of restricted matches.

Let $\hat{R}_{t+1}^{s}=\frac{\phi_{t+1}^{s}+d_{t+1}}{\phi_{t}^{s}}$ denote the measured return on equity between period $t$ and $t+1$, i.e., the return that would be computed by a financial analyst, which includes capital gains and dividend payoffs. Similarly, let $\hat{R}_{t+1}^{b}=\frac{1}{\phi_{t}^{b}}$ denote the measured return on bonds between period $t$ and $t+1$. Also, define $R_{t+1}^{s}=L_{t+1}^{s} \hat{R}_{t+1}^{s}$ and $R_{t+1}^{b}=L_{t+1}^{b} \hat{R}_{t+1}^{b}$, the full (liquidity augmented) returns, as perceived by an agent in the model. With these definitions, and letting $M_{t+1}=\beta \frac{U^{\prime}\left(d_{t+1}\right)}{U^{\prime}\left(d_{t}\right)},(2)$ and (3) lead to

$$
\begin{aligned}
E_{t}\left[M_{t+1}\left(R_{t+1}^{s}-R_{t+1}^{b}\right)\right] & =0 \\
E_{t}\left(M_{t+1} R_{t+1}^{b}-1\right) & =0
\end{aligned}
$$

a pair of statistical restrictions on the marginal rate of substitution and equilibrium asset returns. ${ }^{14}$ Using the definitions of $R_{t+1}^{i}$ and $\hat{R}_{t+1}^{i}$, the unconditional counterparts of (6) and (7) can be written as

$$
\begin{aligned}
E\left[M_{t+1}\left(\hat{R}_{t+1}^{s}-\hat{R}_{t+1}^{b}\right)\right] & =\omega^{e} \\
E\left(M_{t+1} \hat{R}_{t+1}^{b}-1\right) & =\omega^{b}
\end{aligned}
$$

where

$$
\begin{aligned}
\omega^{e} & =E\left\{M_{t+1}\left[\left(L_{t+1}^{b}-1\right) \hat{R}_{t+1}^{b}-\left(L_{t+1}^{s}-1\right) \hat{R}_{t+1}^{s}\right]\right\} \\
\omega^{b} & =-E\left[M_{t+1} \hat{R}_{t+1}^{b}\left(L_{t+1}^{b}-1\right)\right] .
\end{aligned}
$$

\footnotetext{
${ }^{14}$ Note that for the special case of an economy with no liquidity needs (i.e., $L_{t+1}^{s}=L_{t+1}^{b}=1$ ), the measured returns equal the full returns, $\hat{R}_{t+1}^{i}=R_{t+1}^{i}$ for $i=b, s$, and (6) and (7) are equivalent to (2a) and (2b) in Kocherlakota (1996), except for the fact that his expressions assume $U(c)=\frac{c^{1-\sigma}}{1-\sigma}$.
} 
Note that if neither asset provides liquidity services in any state $\left(L_{t+1}^{i}-1=0\right)$, then $\hat{R}_{t+1}^{i}=$ $R_{t+1}^{i}$ and $\omega^{e}=\omega^{b}=0$, so (8) and (9) reduce to (2a') and (2b') in Kocherlakota (1996). ${ }^{15}$

Using actual data, one could estimate the expectations on the left-hand sides of (8) and (9) with the sample means

$$
\begin{aligned}
\hat{\omega}^{e} & =\frac{1}{T} \sum_{t=1}^{T} \beta \frac{U^{\prime}\left(c_{t+1}\right)}{U^{\prime}\left(c_{t}\right)}\left(\hat{R}_{t+1}^{s}-\hat{R}_{t+1}^{b}\right), \\
\hat{\omega}^{b} & =\frac{1}{T} \sum_{t=1}^{T} \beta \frac{U^{\prime}\left(c_{t+1}\right)}{U^{\prime}\left(c_{t}\right)} \hat{R}_{t+1}^{b}-1 .
\end{aligned}
$$

A vast body of work has been devoted to trying to rationalize the finding that for standard parametrizations of preferences, the statistical restrictions $\hat{\omega}^{e}=\hat{\omega}^{b}=0$ are violated by U.S. data. For instance, suppose that $\hat{R}_{t+1}^{s}=\frac{\phi_{t+1}^{s}+d_{t+1}}{\phi_{t}^{s}}$ is constructed using the Standard and Poor's stock index for $\phi_{t}^{s}$ and the real dividends for the Standard and Poor's series for $d_{t}$, and that $\hat{R}_{t}^{b}$ is taken to be the real return on 90-day Treasury bills. Then, if $\beta=0.99$ and $U\left(c_{t}\right)=\frac{c_{t}^{1-\sigma}}{1-\sigma}\left(c_{t}\right.$ is a measure of per capita consumption), one finds $\hat{\omega}^{e}>0$ and $\hat{\omega}^{b}<0$ for "reasonable" values of $\sigma$. (For example, Tables 2 and 3 in Kocherlakota (1996) report $\hat{\omega}^{e}>0$ for $\sigma \leq 8.5$ and $\hat{\omega}^{b}<0$ for $\sigma \geq 0.5$.) The finding that $\hat{\omega}^{e}>0$ constitutes the equity premium puzzle while $\hat{\omega}^{b}<0$ is commonly referred to as the risk-free rate puzzle.

The statistics $\hat{\omega}^{e}$ and $\hat{\omega}^{b}$ that define these puzzles are constructed using the measured returns $\hat{R}_{t+1}^{s}$ and $\hat{R}_{t}^{b}$. But according to the theory developed in the previous sections, agents price assets using the full returns $R_{t+1}^{s}$ and $R_{t+1}^{b}$, that is, the measured returns augmented by their respective liquidity factors, $L_{t+1}^{s}$ and $L_{t+1}^{b}$. To the extent that agents experience liquidity needs in some states, the theory does not imply that $\hat{\omega}^{e}=\hat{\omega}^{b}=0$. In fact, $\omega^{e}$ and $\omega^{b}$, the theoretical counterparts to $\hat{\omega}^{e}$ and $\hat{\omega}^{b}$, will not be zero if the assets are held partly for their exchange value.

From (11) it is clear that $\omega^{b}=0$ if $L_{t+1}^{b}=1$ in all states, i.e., if bonds never provide liquidity services. In this case, condition (9) states that given the measured expected return on bonds, at the margin, the investor must be indifferent between consuming an apple or saving it by using

\footnotetext{
${ }^{15}$ Kocherlakota's (2a') has been divided through by $\beta$ and contains a typo (the marginal rate of substitution appears as $\left(C_{t+1} C_{t}\right)^{-\sigma}$ instead of $\left.\left(C_{t+1} / C_{t}\right)^{-\sigma}\right)$.
} 
it to purchase bonds. In this context, the finding that $\hat{\omega}^{b}<0$ is puzzling because it indicates that the investor could gain by shifting consumption from the future to the present (by saving less in the form of bonds and consuming more today). Or put differently, it appears that the investor is too willing to hold bonds given their relatively low rate of return. But suppose that bonds sometimes offer liquidity services, i.e., suppose that $L_{t+1}^{b}>1$ at least in some state. Then (11) immediately implies $\omega^{b}<0$ : the theory unambiguously predicts that someone testing the agent's Euler equation for the risk-free bill using its measured rate of return $\hat{R}_{t+1}^{b}$ would find that at the margin, this agent can gain from transferring consumption from the future to the present. That is, there would appear to be a risk-free rate puzzle. But this is not a puzzle from the point of view of the model, since the full return on bonds, $L_{t+1}^{b} \hat{R}_{t+1}^{b}$, on average exceeds the measured return $\hat{R}_{t+1}^{b}$, which ignores the liquidity return. Notice that the model is qualitatively consistent with the risk-free rate puzzle for any value of $\theta$, including $\theta=0$, i.e., even without assuming exogenous liquidity differences between equity shares and bonds.

From (10) it is clear that $L_{t+1}^{b}=L_{t+1}^{s}=1$ implies $\omega^{e}=0$. That is, in a world with no liquidity needs, the measured returns $\hat{R}_{t+1}^{s}$ and $\hat{R}_{t+1}^{b}$ have to be such that at the margin, the agent cannot gain by substituting from bonds into stocks, or vice versa. In this context, the finding that $\hat{\omega}^{e}>0$ is puzzling because it indicates that the investor could gain by shifting his portfolio from bonds to stocks. Or put differently, it appears that the investor is too willing to hold bonds vis-à-vis stocks given that, according to the measured returns, equity pays such a large premium over bonds. But suppose that $\theta=1$. Then $(10)$ becomes $\omega^{e}=$ $E\left[M_{t+1}\left(L_{t+1}^{b}-1\right) \hat{R}_{t+1}^{b}\right] \geq 0$, with strict inequality if bonds provide liquidity in some state. In this case, according to the theory, someone testing the investor's Euler equation for the measured excess returns would find that, at the margin, the agent can gain from disinvesting in bonds and investing more in equity. There would appear to be an equity-premium puzzle because if there are liquidity needs but equity shares cannot be used to satisfy them, equity must pay an illiquidity premium over bonds, and this shows up as a wedge $\omega^{e}>0$.

The model can generate $\omega^{e}>0$ even if $\theta=0$, i.e., even if no advantage in exchange 
is given to bonds over stocks. In this case, $L_{t+1}^{b}=L_{t+1}^{s}=L_{t+1}$, and (10) becomes $\omega^{e}=$ $E\left[M_{t+1}\left(L_{t+1}-1\right)\left(\hat{R}_{t+1}^{b}-\hat{R}_{t+1}^{s}\right)\right]$. The wedge $\omega^{e}$ is a weighted average of the measured return differentials between bonds and equity over states. The sign of $\omega^{e}$ is ambiguous in general. But, for example, suppose there is a high- and a low-growth state and that $\left(\hat{R}_{t+1}^{b}-\hat{R}_{t+1}^{s}\right)$ is positive in the low state and negative in the high state. Then if the "weight" $M_{t+1}\left(L_{t+1}-1\right)$ tends to be larger in the low state, the model will generate $\omega^{e}>0$. Thus, even without legal or institutional restrictions that give bonds an advantage over equity as a medium of exchange, the theory can still be consistent with an equity-premium puzzle. In fact, in the calibration of Section 4 , I will find that for some values of risk aversion, the model with $\theta=0$ conforms with this reasoning, and as a result, it indeed implies $\omega^{e}>0$.

To summarize, the model with liquidity needs is always (for any $\theta$ ) qualitatively consistent with the risk-free rate puzzle. In addition, the model may also help to rationalize the equitypremium puzzle - even if $\theta=0$ - provided that measured returns, liquidity returns, and the marginal rate of substitution covary in certain ways.

\section{Quantitative analysis}

I will follow the original formulation in Mehra and Prescott (1985) and let the utility over apples and coconuts be $U(c)=u(c)=\frac{c^{1-\sigma}}{1-\sigma}$, with $0<\sigma<\infty$. For this parametrization, $q\left(z ; \kappa d_{t+1}\right)$, i.e., the quantity of coconuts traded in a meeting where the buyer can pay from a portfolio of end-of-subperiod real value ${ }^{16} z$ when the aggregate dividend is $d_{t+1}$, is given by

$$
q\left(z ; \kappa d_{t+1}\right)= \begin{cases}\epsilon \kappa d_{t+1} & \text { if } z \geq g\left(\kappa d_{t+1}\right) \\ (1+\epsilon) \kappa d_{t+1}-\left\{\left[(1+\epsilon) \kappa d_{t+1}\right]^{1-\sigma}-(1-\sigma) z\right\}^{\frac{1}{1-\sigma}} & \text { if } z<g\left(\kappa d_{t+1}\right)\end{cases}
$$

where $g\left(\kappa d_{t+1}\right)=\left[(1+\epsilon)^{1-\sigma}-1\right] \frac{\left(\kappa d_{t+1}\right)^{1-\sigma}}{1-\sigma}$. (If $\sigma=1$, then $q\left(z ; \kappa d_{t+1}\right)=\epsilon \kappa d_{t+1}$ if $z \geq$ $\ln (1+\varepsilon)$, and $q\left(z ; \kappa d_{t+1}\right)=\left(1-e^{-z}\right)(1+\epsilon) \kappa d_{t+1}$ otherwise. $)$

\footnotetext{
${ }^{16} \mathrm{By}$ "real value" here, I mean real value in terms of marginal utility of fruit. For example, in a period when the aggregate dividend is $d_{t}$, the "real value" of a portfolio $\mathbf{a}_{t}=\left(a_{t}^{s}, a_{t}^{b}\right)$ is $\boldsymbol{\lambda}_{t} \mathbf{a}_{t}=\lambda_{t}^{s} a_{t}^{s}+\lambda_{t}^{b} a_{t}^{b}$, where $\lambda_{t}^{b}=U^{\prime}\left(d_{t}\right)$ and $\lambda_{t}^{s}=U^{\prime}\left(d_{t}\right)\left(\phi_{t}^{s}+d_{t}\right)$.
} 
Hereafter, I specify that the government chooses the stock of bonds according to a policy rule $B_{t+1}=f\left(d_{t}, x_{t}\right)$. At any date, the variables $\left(d_{t}, x_{t}\right)$ are sufficient for predicting the subsequent evolution of the economy. So in a recursive equilibrium (one where all equilibrium values are invariant functions of the state), one can think of the state of the economy as being the pair $(d, i)$ if $d_{t}=d$ and $x_{t}=\gamma_{i}$. With this convention, asset prices can be written as $\phi^{s}(d, i)$ and $\phi^{b}(d, i)$. To guarantee that the ratio of government debt to GDP is stationary, let $f\left(d_{t}, x_{t}\right)=$ $\hat{B} E\left[d_{t+1} \mid\left(d_{t}, i\right)\right]$ if $x_{t}=\gamma_{i}$, where $\hat{B} \geq 0$. Thus, given that $x_{t}=\gamma_{i}, B_{t+1}=f\left(d_{t}, x_{t}\right)=B_{i} d_{t}$, where $B_{i}=\hat{B} \sum_{j=1}^{n} \mu_{i j} \gamma_{j}$. Restricting attention to equilibria that are stationary in growth rates amounts to focusing on equilibria where share prices are homogeneous of degree one in $d$, and can therefore be written as $\phi^{s}(d, j)=\phi_{j}^{s} d$, where $\phi_{j}^{s}$ is a constant. In turn, this implies that $\phi^{b}(d, i)$ is independent of $d$, so one can write $\phi_{i}^{b}=\phi^{b}(d, i)$. Now (2)-(5) reduce to

$$
\begin{aligned}
\phi_{i}^{s} & =\beta \sum_{j} \mu_{i j} \gamma_{j}^{1-\sigma} L_{i j}^{s}\left(\phi_{j}^{s}\right)\left(1+\phi_{j}^{s}\right) \\
\phi_{i}^{b} & =\beta \sum_{j} \mu_{i j} \gamma_{j}^{-\sigma} L_{i j}^{b}\left(\phi_{j}^{s}\right),
\end{aligned}
$$

for $i=1, \ldots, n$, with

$$
\begin{aligned}
& L_{i j}^{s}\left(\phi_{j}^{s}\right)=1+\alpha(1-\theta) \max \left\{\left[\frac{2}{\left[(1+\epsilon)^{1-\sigma}+\frac{(\sigma-1)}{\kappa^{1-\sigma}}\left(1+\phi_{j}^{s}+\frac{B_{i}}{\gamma_{j}}\right)\right]^{\frac{1}{1-\sigma}}}-1\right]^{-\sigma}-1,0\right\} \\
& L_{i j}^{b}\left(\phi_{j}^{s}\right)=L_{i j}^{s}\left(\phi_{j}^{s}\right)+\alpha \theta \max \left\{\left[\frac{2}{\left[(1+\epsilon)^{1-\sigma}+\frac{(\sigma-1)}{\kappa^{1-\sigma}} \frac{B_{i}}{\gamma_{j}}\right]^{\frac{1}{1-\sigma}}}-1\right]^{-\sigma}-1,0\right\} .
\end{aligned}
$$

Let $\left(\bar{\mu}_{i}\right)_{i=1}^{n}$ denote the vector of stationary probabilities on $i$, i.e., the vector that solves $\bar{\mu}=\mu^{\prime} \bar{\mu}$, with $\sum_{i} \bar{\mu}_{i}=1$, where $\mu$ is the Markov matrix $\left[\mu_{i j}\right]$ and $\mu^{\prime}=\left[\mu_{j i}\right]$. Then,

$$
\begin{aligned}
\sum_{i} \sum_{j} \bar{\mu}_{i} \mu_{i j} \beta \gamma_{j}^{-\sigma}\left(\hat{R}_{i j}^{s}-\hat{R}_{i}^{b}\right) & =\omega^{e} \\
\sum_{i} \sum_{j} \bar{\mu}_{i} \mu_{i j}\left(\beta \gamma_{j}^{-\sigma} \hat{R}_{i}^{b}-1\right) & =\omega^{b}
\end{aligned}
$$


are the analogues of (8) and (9), where $\hat{R}_{i j}^{s}=\frac{\left(1+\phi_{j}^{s}\right) \gamma_{j}}{\phi_{i}^{s}}, \hat{R}_{i}^{b}=1 / \phi_{i}^{b}$, and

$$
\begin{aligned}
\omega^{e} & =\sum_{i} \sum_{j} \bar{\mu}_{i} \mu_{i j} \beta \gamma_{j}^{-\sigma}\left\{\left[L_{i j}^{b}\left(\phi_{j}^{s}\right)-1\right] \hat{R}_{i}^{b}-\left[L_{i j}^{s}\left(\phi_{j}^{s}\right)-1\right] \hat{R}_{i j}^{s}\right\} \\
\omega^{b} & =-\sum_{i} \sum_{j} \bar{\mu}_{i} \mu_{i j} \beta \gamma_{j}^{-\sigma}\left[L_{i j}^{b}\left(\phi_{j}^{s}\right)-1\right] \hat{R}_{i}^{b} .
\end{aligned}
$$

The average measured returns on equity and the bond are

$$
\hat{R}^{s}=\sum_{i} \sum_{j} \bar{\mu}_{i} \mu_{i j} \hat{R}_{i j}^{s} \quad \text { and } \quad \hat{R}^{b}=\sum_{i} \bar{\mu}_{i} \hat{R}_{i}^{b}
$$

and the equity premium is $\pi=\hat{R}^{s}-\hat{R}^{b}$.

The liquidity factors (16) and (17) reflect the fact that the trading constraints in the bargaining problem, $\mathbf{p}^{k} \leq \mathbf{a}$ for $k=1,2$ (where $k=1$ denotes a restricted match, and $k=2$ an unrestricted match), may bind in some states and not in others. Suppose that the current realization of the growth rate is $\gamma_{i}$. Then from (16), it is clear that the set of next-period states in which the trading constraint binds in unrestricted matches is

$$
\Omega\left(B_{i}\right)=\left\{j \in\{1, \ldots, n\}: 1+\phi_{j}^{s}+\frac{B_{i}}{\gamma_{j}}<\frac{\kappa^{1-\sigma}}{\sigma-1}\left[1-(1+\epsilon)^{1-\sigma}\right]\right\} .
$$

Hence, an economy with $\theta \in[0,1)$ has $L_{i j}^{s}>1$ if and only if $j \in \Omega\left(B_{i}\right)$. Similarly, given that the current period growth rate is $\gamma_{i}$, the set of next-period states in which the liquidity constraint binds in restricted matches is

$$
\Omega_{\theta}\left(B_{i}\right)=\left\{j \in\{1, \ldots, n\}: \frac{B_{i}}{\gamma_{j}}<\frac{\kappa^{1-\sigma}}{\sigma-1}\left[1-(1+\epsilon)^{1-\sigma}\right]\right\} .
$$

Naturally, $\Omega\left(B_{i}\right) \subseteq \Omega_{\theta}\left(B_{i}\right)$; i.e., if the liquidity constraint binds in unrestricted trades, it also binds in restricted trades. If $\Omega_{\theta}\left(B_{i}\right)=\varnothing$ for all $i$, then $L_{i j}^{s}\left(\phi_{j}^{s}\right)=L_{i j}^{b}\left(\phi_{j}^{s}\right)=1$ for all $i$ and $j$, $\omega^{e}=\omega^{b}=0$, and the model reduces to Mehra and Prescott (1985). The following example can help build some intuition.

Example 1 Suppose $\mu_{i j}=\mu_{j}$ for all $i$, let $\Delta \equiv \beta \sum_{j} \mu_{j} \gamma_{j}^{1-\sigma}<1, \bar{\gamma}=\max _{i} \gamma_{i}$, and $\underline{\gamma}=$ $\min _{i} \gamma_{i}$. Let $\bar{B}=\left\{\frac{\kappa^{1-\sigma}}{\sigma-1}\left[1-(1+\epsilon)^{1-\sigma}\right]-\frac{1}{1-\Delta}\right\} \bar{\gamma}$. Then, $\phi_{i}^{s}=\phi^{s}, \phi_{i}^{b}=\phi^{b}$, and $B_{i}=B$ for 
all $i$, and: (a) For $B \geq \bar{B}$, we have $\phi^{s}=\frac{\Delta}{1-\Delta}, \phi^{b}=\beta \sum_{j} \mu_{j} \gamma_{j}^{-\sigma}$, and $\Omega_{\theta}=\Omega=\varnothing$; i.e., $L_{j}^{s}\left(\phi^{s}\right)=L_{j}^{b}\left(\phi^{s}\right)=1$ for all $j$. (b) For $B<\bar{B}, \phi^{s}=\phi^{*}$, where $\phi^{*}$ is the unique solution to $\frac{\phi}{1+\phi}=\beta \sum_{j} \mu_{j} \gamma_{j}^{1-\sigma} L_{j}^{s}(\phi)$, and given $\phi^{*}, \phi^{b}=\beta \sum_{j} \mu_{j} \gamma_{j}^{-\sigma} L_{j}^{b}\left(\phi^{*}\right)$. In this case, $\Omega_{\theta} \neq \varnothing$ and $\Omega \neq \varnothing$, so $L_{j}^{b}\left(\phi^{*}\right)>L_{j}^{s}\left(\phi^{*}\right)$ for (at least) some $j$, and $L_{i}^{s}\left(\phi^{*}\right)>1$ for (at least) some i. To provide a full characterization, let $\bar{B}_{\theta}=\frac{\kappa^{1-\sigma}}{\sigma-1}\left[1-(1+\epsilon)^{1-\sigma}\right] \bar{\gamma}, \underline{B}_{\theta}=(\underline{\gamma} / \bar{\gamma}) \bar{B}_{\theta}$, and $\underline{B}=\left\{\frac{\kappa^{1-\sigma}}{\sigma-1}\left[1-(1+\epsilon)^{1-\sigma}\right]-\left(1+\phi^{*}\right)\right\} \underline{\gamma}$. (It is easy to see that in general, $\underline{B}_{\theta}<\bar{B}_{\theta}, \underline{B}<\bar{B}$, $\bar{B}<\bar{B}_{\theta}$, and $\underline{B}<\underline{B}_{\theta}$.) Let $\Omega_{\theta}^{c}$ denote the complement of $\Omega_{\theta}$ and $\Omega^{c}$ the complement of $\Omega$. Then it can be shown that (i) $\Omega_{\theta}^{c}=\varnothing$ iff $B<\underline{B}_{\theta}$, and $\Omega_{\theta}=\varnothing$ iff $\bar{B}_{\theta} \leq B$; and (ii) that $\Omega^{c}=\varnothing$ iff $B<\underline{B}$, and $\Omega=\varnothing$ iff $\bar{B} \leq B$.

\subsection{Calibration}

The three basic types of parameters are those that define preferences ( $\beta$ and $\sigma$ ), those that define technology $\left(\alpha, \epsilon, \kappa\right.$, and the elements of $\left[\mu_{i j}\right]$ and $\left.\left[\gamma_{i}\right]\right)$, and $\hat{B}$, which defines government policy. In the formulations that assume differential liquidity, there is also $\theta$, the probability that a randomly drawn trading partner will not accept shares as a means of payment in a decentralized exchange. ${ }^{17}$

I follow Mehra and Prescott (1985) and assume that the growth rate of the aggregate fruit endowment follows a two-state Markov chain with $\gamma_{1}=\bar{\gamma}+\delta, \gamma_{2}=\bar{\gamma}-\delta, \mu_{11}=\mu_{22}=\mu$, and $\mu_{12}=\mu_{21}=1-\mu$, and set $\bar{\gamma}=1.018, \delta=0.036$, and $\mu=0.43 .{ }^{18}$ Next, I propose a calibration procedure for the remaining parameters. Since the main goal here is to explore the quantitative relevance of the liquidity channel (rather than to devise a definitive calibration strategy for this class of models), I will also consider various alternative parametrizations for the variables that were not in Mehra and Prescott's original analysis.

\footnotetext{
${ }^{17}$ There are also the parameters associated with the utility of general goods and the disutility of work, but they are irrelevant for asset prices within the class of equilibria studied here. (See Section A.2 in the appendix, in particular footnote 39 , for details.)

${ }^{18}$ Mehra and Prescott selected these parameter values so that the average growth rate of per capita consumption, the standard deviation of the growth rate of per capita consumption, and the first-order serial correlation of this growth rate (all with respect to the model's invariant distribution) matched the sample values for the U.S. economy between 1889 and 1978. The same is true here.
} 
The strategy for calibrating $\kappa$ consists of selecting the value for which the fraction of GDP that corresponds to production units ("trees") that have outstanding tradeable equity shares in the model matches the sample value for the U.S. economy. Since this statistic is not readily available, I have instead guided the choice of $\kappa$ by using the gross value added of the nonfinancial corporate business sector as a share of GDP. In the U.S. National Income and Product Accounts (NIPA), this number is slightly above 0.5 for the fifty-seven-year period $1947-2004 .{ }^{19}$ Not all businesses have outstanding tradeable equity, so this amounts to an upper bound. With this in mind, I target 0.4 as a benchmark, which results in $\kappa=0.5 .^{20}$ In the appendix, I also report results for different targets, both larger and smaller.

The policy parameter $\hat{B}$ determines the size of the stock of government-issued assets that can be used as a medium of exchange, and hence the extent to which agents may experience liquidity needs in decentralized trades. Government bonds are the only such asset in the model, but in the United States, agents may use other government-issued assets (and claims to these assets) to satisfy their liquidity needs. With this in mind, I constructed a new empirical monetary aggregate, M1*, which augments and adjusts the standard M1 measure. The measure M1* differs from M1 in two ways. First, it includes Treasury bills in circulation. And second, it makes an adjustment for the amounts of M1 and Treasury bills outstanding that are held outside the United States. ${ }^{21}$ The ratio of M1* to annual GDP was about 0.3 in 1959, declined

\footnotetext{
${ }^{19}$ The data for Gross Value Added of Domestic Corporate Business and GDP are from NIPA, Tables 1.1.4 and 1.15 , respectively.

${ }^{20}$ In the model, the fraction of GDP produced by trees that have outstanding, tradeable equity shares is $\frac{1}{2+\kappa}$. In period $t$, there is an endowment $\kappa d_{t}$ of fruit in the first subperiod and an endowment $d_{t}$ in the second, so the total endowment of fruit in period $t$ is $(1+\kappa) d_{t}$. The output of general goods in the second subperiod is $n^{*} d_{t}$, where $n^{*}=A^{-1 / \sigma}$. (See Section A.2 in the appendix for details.) Multiplying this quantity by the relative price of general goods in terms of fruit, $A$, yields the value of production of general goods expressed in terms of fruit, $A n^{*} d_{t}$. So real GDP (in terms of fruit) is $\left(1+\kappa+A n^{*}\right) d_{t}$, or just $(2+\kappa) d_{t}$, given $A=1$.

${ }^{21}$ Data for M1 are from the Board of Governors of the Federal Reserve System (Money Stock Measures, Release H.6). The amount of Treasury bills outstanding for 1959-2004 was obtained from the Bureau of the Public Debt (Monthly Statement of the Public Debt, Table I: Summary of Treasury Securities Outstanding). The Flow of Funds Accounts of the United States compiled by the Board of Governors of the Federal Reserve System provides estimates of the portion of checkable deposits and currency and of Treasury securities that is held by the rest of the world (Table L.204, line 20, and Table L.209, line 11, respectively). The series for M1 was adjusted by assuming that the proportion that is held domestically is the same as the proportion of currency and checkable deposits that is held domestically. Similarly, the stock of outstanding Treasury bills held domestically was estimated by assuming that the fraction of Treasury bills held by the rest of the world is the same as the
} 
through 1979, hovered around 0.2 until 1995, and has been about 0.15 since then. So summing up, this ratio has been in the interval $[0.15,0.3]$ over the last 40 years. I use 0.3 as a benchmark target - a conservative choice, since the magnitude of the liquidity factor is decreasing in the value of this ratio. This choice, together with the benchmark value for $\kappa$, implies $\hat{B}=0.75$. In the appendix, I will also report results for different values of $\hat{B}$.

The parameters $\alpha$ and $\epsilon$ index the agent's ability and desire to engage in bilateral exchange, respectively. If $\alpha=0$, the economy is just a sequence of Walrasian markets, and assets are valued only for their intrinsic payoffs but not for their role as media of exchange: in this case, the model reduces to the one studied by Mehra and Prescott (1985). If $\epsilon=0$, each agent has the same endowment every first subperiod, so there are no benefits from trade in any bilateral meeting and therefore no role for a medium of exchange. In this case, asset prices and returns will again be just as in the economy studied by Mehra and Prescott.

The choice of $\epsilon$ has implications for consumption inequality. In the baseline calibration, I set $\epsilon=0.8$, which implies that the variance of the log of total (including general goods and fruit in both subperiods) per capita consumption is $0.013 .{ }^{22}$ In the appendix (Section A.4), I will also explore the implications of higher and lower values for $\epsilon$. Note that $\alpha=\hat{\alpha} / 4$, where $\hat{\alpha}$ is the probability that the agent finds an opportunity to trade in the decentralized market. I set $\hat{\alpha}=1$ in the baseline, which basically says that every agent has a trading opportunity in the decentralized market over the course of the year. However, only the products $\alpha \theta$ and $\alpha(1-\theta)$ matter for asset prices and returns, and I will be experimenting extensively with $\theta$. In the appendix (Section A.4), I verify the robustness of the main findings to changes in the

fraction of Treasury securities held by the rest of the world. The series for M1* was constructed by adding the estimated domestic holdings of Treasury bills to the estimated stock of M1 that is held domestically.

${ }^{22}$ This number is rather low relative to the values of about 0.15 that Krueger and Perri (2005) report for the within-group (i.e., conditioning on education, age, sex, experience, occupation, region of residence) variance of the $\log$ of per capita consumption in the United States. The cross-sectional variance of the log of total consumption is low even for values of $\epsilon$ close to 1 . This is so because in the model, there is only inequality in consumption of fruit in the first subperiod, and this is only a fraction of total consumption (in addition, all agents also consume the same amounts of fruit and general goods in the second subperiod). For the various parametrizations, I have also computed the variance of the log of per capita consumption in the first subperiod only - and found it to be in line with the estimates of Krueger and Perri (2005). 
values of $\hat{B}, \kappa$, and $\varepsilon$, the parameters that were not in Mehra and Prescott (1985).

The nature of Mehra and Prescott's test of the theory was to experiment with different values of $\beta$ and $\sigma$ to find the combinations of these parameters for which the model's average risk-free rate and equity premium matched those observed for the U.S. economy. (Table 1, taken from Mehra and Prescott (2003), reports the basic facts according to various data sets.) In the next section I will carry out a similar exercise, except that I will not experiment with $\beta$, which is set to 0.99 throughout. I will first consider the economy with no exogenous liquidity differences between equity shares and bonds, and assess the model's ability to produce bond returns and equity premia that resemble those observed for the U.S. economy. I will then go on to consider the specification with exogenous liquidity differences where shares are not accepted as a means of payment in a fraction $\theta$ of decentralized exchanges. For this specification, the question I pose is: how large does $\theta$ (the relative illiquidity of equity) need to be for the model to generate an average risk-free rate of $1 \%$ and an average equity premium that matches the one observed in the U.S. economy?

\begin{tabular}{lccc}
\hline \hline Data Set & $\begin{array}{c}\text { \% Real Return } \\
\text { on a Market } \\
\text { Index (Mean) }\end{array}$ & $\begin{array}{c}\text { \% Real Return on a } \\
\text { Relatively Riskless } \\
\text { Security (Mean) }\end{array}$ & $\begin{array}{c}\text { \% Equity Premium } \\
\text { (Mean) }\end{array}$ \\
\hline 1889-1978 (Mehra-Prescott, 1985) & 6.98 & 0.80 & 6.18 \\
1889-2000 (Mehra-Prescott, 2003) & 8.06 & 1.14 & 6.92 \\
1926-2000 (Ibbotson Associates, 2001) & 8.80 & 0.40 & 8.40 \\
1871-1999 (Shiller, 1990) & 6.99 & 1.74 & 5.25 \\
1802-1998 (Siegel, 1998) & 7.00 & 2.90 & 4.10 \\
\hline
\end{tabular}

Table 1: U.S. equity premium from different data sets

\subsection{Results}

Table 2 reports the average percentage return on equity, the average percentage return on the bond, and their difference (the equity premium) in the Mehra-Prescott economy for values of $\sigma$ ranging from 1 to 10. For these "reasonable" values of $\sigma$, the risk-free rate is too high and the equity premium too low. Table 3 corresponds to the benchmark calibration of the 
basic model augmented to allow for a liquidity motive, as outlined in the previous sections. (Table 3 assumes $\theta=0$, i.e., no exogenous liquidity differences between equity and bonds.) The last column of each of these tables reports the pair of wedges $\left(\omega^{e}, \omega^{b}\right)$ to the agent's Euler equations (see (18) and (19)). These wedges are zero if agents have enough liquidity to conduct decentralized transactions in all states of the world, or if the liquidity channel is shut off, as in the Mehra-Prescott economy.

\begin{tabular}{ccccc}
\hline \hline$\sigma$ & $\begin{array}{c}\text { Equity } \\
\text { Return }\end{array}$ & $\begin{array}{c}\text { Bond } \\
\text { Return }\end{array}$ & $\begin{array}{c}\text { Equity } \\
\text { Premium }\end{array}$ & $\begin{array}{c}\text { EE wedges } \\
\omega^{e}, \omega^{b}\end{array}$ \\
\hline 1 & 2.83 & 2.70 & 0.13 & 0,0 \\
2 & 4.58 & 4.30 & 0.28 & 0,0 \\
3 & 6.27 & 5.79 & 0.48 & 0,0 \\
4 & 7.89 & 7.18 & 0.71 & 0,0 \\
5 & 9.42 & 8.45 & 0.97 & 0,0 \\
6 & 10.88 & 9.62 & 1.26 & 0,0 \\
7 & 12.24 & 10.67 & 1.57 & 0,0 \\
8 & 13.52 & 11.60 & 1.92 & 0,0 \\
9 & 14.70 & 12.41 & 2.29 & 0,0 \\
10 & 15.79 & 13.10 & 2.69 & 0,0 \\
\hline
\end{tabular}

Table 2: The Mehra-Prescott economy

\begin{tabular}{ccccc}
\hline \hline$\sigma$ & $\begin{array}{c}\text { Equity } \\
\text { Return }\end{array}$ & $\begin{array}{c}\text { Bond } \\
\text { Return }\end{array}$ & $\begin{array}{c}\text { Equity } \\
\text { Premium }\end{array}$ & $\begin{array}{c}\text { EE wedges } \\
\omega^{e}, \omega^{b}\end{array}$ \\
\hline 1 & 2.83 & 2.70 & 0.13 & 0,0 \\
2 & 4.58 & 4.30 & 0.28 & 0,0 \\
3 & 6.27 & 5.79 & 0.48 & 0,0 \\
4 & 7.89 & 7.18 & 0.71 & 0,0 \\
5 & 9.42 & 8.45 & 0.97 & 0,0 \\
6 & 10.88 & 9.62 & 1.26 & 0,0 \\
7 & 12.24 & 10.67 & 1.57 & 0,0 \\
8 & 8.80 & 6.76 & 2.04 & $.0010,-.0435$ \\
9 & 5.97 & 3.54 & 2.43 & $.0014,-.0790$ \\
10 & 4.35 & 1.50 & 2.85 & $.0017,-.1027$ \\
\hline
\end{tabular}

Table 3: Benchmark economy with $\theta=0$

The first thing to note is that the first seven rows in Table 3 are identical to the corresponding rows in Table 2: the economy behaves exactly as the Mehra-Prescott economy up to $\sigma=7$. By 
the time $\sigma$ reaches 8 , equilibrium asset prices and returns begin to differ across both economies. For example, in Table 2, when the curvature parameter goes from 7 to 8 , the return on equity rises from $12.24 \%$ to $13.52 \%$, and the bond return rises from $10.67 \%$ to $11.6 \%$. In contrast, in the economy of Table 3, the equity and bond returns fall from $12.24 \%$ to $8.8 \%$, and from $10.67 \%$ to $6.76 \%$, respectively. The two economies behave differently for higher values of $\sigma$ because agents face binding liquidity constraints in bilateral trades for high values of $\sigma$, but not for low values.

To see why this is the case, let $\Omega\left(B_{i}\right)$ denote the set of states for which the liquidity constraints bind in the economy with $\theta=0$, in a period when the outstanding stock of bonds is $\frac{B_{i}}{\gamma_{j}} d$. From the analysis in the previous sections, we know that

$$
\Omega\left(B_{i}\right)=\left\{j \in\{1,2\}: 1+\phi_{j}^{s}+\frac{B_{i}}{\gamma_{j}}<\frac{\kappa^{1-\sigma}}{\sigma-1}\left[1-(1+\epsilon)^{1-\sigma}\right]\right\} .
$$

Recall that $\left(1+\phi_{j}^{s}+\frac{B_{i}}{\gamma_{j}}\right) d$ is the real (value in terms of fruit of the) equilibrium portfolio at the beginning of the first subperiod when the state is $(d, j)$ and the outstanding stock of bonds is $\frac{B_{i}}{\gamma_{j}} d$, and hence $\left(1+\phi_{j}^{s}+\frac{B_{i}}{\gamma_{j}}\right) d^{1-\sigma}$ is the "price" of this portfolio in terms of marginal utility of fruit. The definition of the set $\Omega\left(B_{i}\right)$ states that the buyer's liquidity constraint binds if this value of her portfolio falls short of $u[(1+\epsilon) \kappa d]-u(\kappa d)=\frac{\kappa^{1-\sigma}}{\sigma-1}\left[1-(1+\epsilon)^{1-\sigma}\right] d^{1-\sigma}$, namely, the amount of utility she has to transfer to the seller for him to be willing to consume $\kappa d$ instead of $(1+\epsilon) \kappa d$ in the first subperiod. In the benchmark parametrization, it can be shown that $\frac{\kappa^{1-\sigma}}{\sigma-1}\left[1-(1+\epsilon)^{1-\sigma}\right]$ is strictly increasing in $\sigma$. This means that, keeping $\phi_{j}^{s}$ constant, the set $\Omega$ is nondecreasing in $\sigma$. Of course, in the general equilibrium $\sigma$ also affects equity prices $\phi_{j}^{s}$, but for the baseline parametrization, the direct effect that tends to tighten the liquidity constraint quantitatively dominates the indirect effect that operates through the price of equity (which in general tends to loosen the liquidity constraint, since equity prices typically rise with $\sigma$ when the liquidity constraints are not always slack).

To be more precise, for the economy reported in Table 3 , there is a $\sigma_{h} \approx 7.153$ such that for all $i \in\{1,2\}$, we have $\Omega\left(B_{i}\right)=\varnothing$ for $\sigma \leq \sigma_{h}$ and $\Omega\left(B_{i}\right)=\{1,2\}$ for $\sigma>\sigma_{h}$. That is, for 
$\sigma>\sigma_{h}$, buyers in the decentralized market face binding liquidity constraints for all realizations of the aggregate endowment process and every outstanding stock of bonds implied by the government's policy rule. When liquidity constraints bind, assets help relax those constraints, and this additional "liquidity service" they provide is reflected in asset prices and their measured returns - the asset returns as they are conventionally measured. When the liquidity constraints bind, asset prices rise, and their measured, intrinsic returns fall with $\sigma$. The reason is that the liquidity constraint becomes progressively tighter with larger values of $\sigma$, so agents have an additional incentive to hold assets: they help relax liquidity constraints. This means thatrelative to a world with no liquidity - investors no longer require such large expected measured returns to be willing to hold those assets. (Conversely, in the Mehra-Prescott economy of Table 2 , asset prices are strictly decreasing and returns strictly increasing in $\sigma$, at least for reasonable values, e.g., $\sigma \leq 20$.)

If we compare the third columns of Tables 2 and 3 , it is clear that, for $\sigma>\sigma_{h}$, the equity premium is only slightly higher in the model with liquidity and $\theta=0$, relative to the MehraPrescott economy. Take the row corresponding to $\sigma=10$, for instance: the standard model generates a premium of $2.69 \%$ and the model with liquidity a premium of $2.85 \%$. The big difference is in the actual asset returns that compose this premium. In the standard model the $2.69 \%$ is the difference between an equity return of $15.79 \%$ and a risk-free rate of $13.10 \%$ - both much too high relative to the values reported in Table 1 . In contrast, the $2.85 \%$ premium in the model with liquidity is the difference between an equity return of $4.35 \%$ and a risk-free rate of $1.5 \%$. The former is rather low, but the latter is within the range of estimates reported in Table 1. All this is illustrated in Figure 1.

The last column of Tables 2 and 3 reports the "wedges" to the Euler equations defined in (18) and (19). This column can be used as an indicator of when the liquidity constraints bind and when they do not. In Table 3 , for $\sigma \leq \sigma_{h}$, i.e., when these constraints are slack in all states, both wedges equal zero, and the equilibrium asset prices and returns coincide with those in the Mehra-Prescott economy. For $\sigma>\sigma_{h}$ the liquidity constraints bind, so these wedges become 

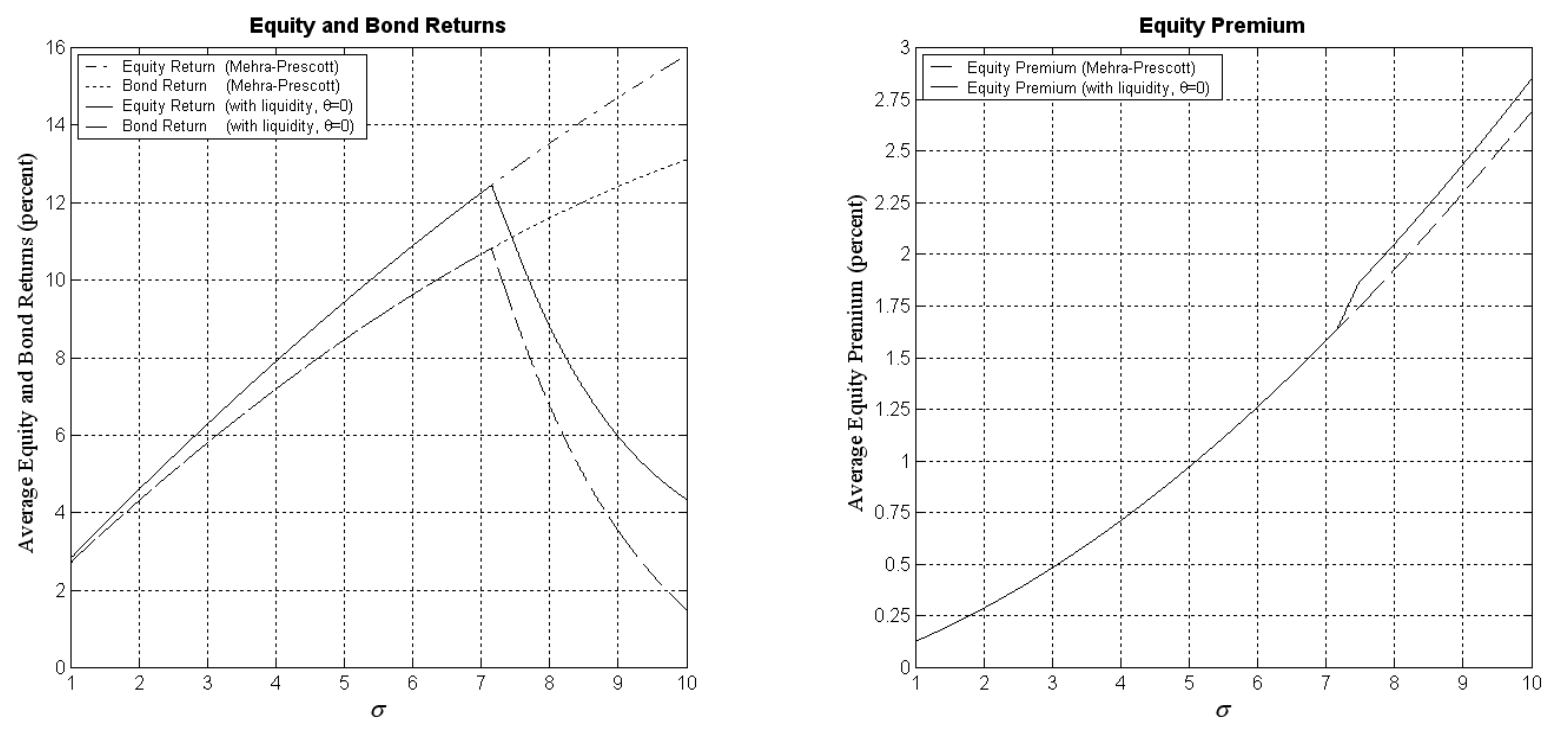

Figure 1: Returns for Mehra-Prescott and for the economy with liquidity $(\theta=0)$

nonzero. Since Table 3 assumes $\theta=0$, we have $L_{i j}^{b}\left(\phi_{j}^{s}\right)=L_{i j}^{s}\left(\phi_{j}^{s}\right)$, and therefore (see (18)) the wedge $-\omega^{e}$ is a weighted average of $\left(\hat{R}_{i j}^{s}-\hat{R}_{i}^{b}\right)$, the state-by-state equity premium. Since the average equity premium is modest and the "weight" $\beta \gamma_{j}^{-\sigma} L_{i j}$ is large in the low-growth state, when $\left(\hat{R}_{i j}^{s}-\hat{R}_{i}^{b}\right)<0$, and small in the high-growth state, when $\left(\hat{R}_{i j}^{s}-\hat{R}_{i}^{b}\right)>0$, the model delivers $\omega^{e}>0 .{ }^{23}$ The wedge $-\omega^{b}$ is a weighted average of the risk-free rate, $\hat{R}_{i}^{b}$, so we have $\omega^{b}<0(\operatorname{see}(19))$.

As mentioned in Section 3, a large body of empirical work in asset pricing specifies and tests the sample counterpart to the moment restriction implied by the Euler equation of candidate structural models (e.g., Hansen and Singleton (1982)). The wedges $\omega^{e}$ and $\omega^{b}$ in the last column of Table 3 can be thought of as the theoretical counterparts of the sample means reported in Kocherlakota (1996) (Tables 2 and 3, pp. 50-51). Interestingly, even with $\theta=0$, the model is able to replicate the signs of these wedges. Quantitatively, however, $\omega^{e}$ is too small relative to

\footnotetext{
${ }^{23}$ For $\sigma=10$, for example, $\hat{R}_{1}^{b}=0.96, \hat{R}_{2}^{b}=1.07, \hat{R}_{11}^{s}=1.076, \hat{R}_{21}^{s}=1.169, \hat{R}_{12}^{s}=0.92$, and $\hat{R}_{22}^{s}=1$. Note that $\hat{R}_{i 1}^{s}-\hat{R}_{i}^{b}>0$ and $\hat{R}_{i 2}^{s}-\hat{R}_{i}^{b}<0$ for $i=1,2$. The liquidity factors are $L_{11}=1.0726, L_{21}=1.0725$, $L_{12}=1.1355$, and $L_{22}=1.1353$.
} 
the sample means that Kocherlakota reports in his Table $2 .{ }^{24}$ Conversely, the value of $\omega^{b}$ for $\sigma$ ranging from 7 to 10 is roughly in line with magnitudes of the sample means that Kocherlakota reports in his Table 3 - an indication that, even with $\theta=0$, the model is able to rationalize the risk-free rate puzzle.

The risk-free rate remains low for high risk aversion because assets, and in particular bonds, yield a liquidity return which agents take into account when choosing their portfolios, but financial analysts don't include in their calculations of actual measured returns. In the usual Mehra-Prescott economy, the risk-free rate puzzle arises because at relatively high levels of $\sigma$, the equilibrium must offer agents in a growing economy a high return on bonds for them to be willing to hold them. From the optic of the model with liquidity needs, at higher levels of $\sigma$ the equilibrium compensates agents for holding bonds (and since $\theta=0$, equity) with a high overall return composed of a relatively high liquidity return and a relatively low "measured" return of about $1.5 \%$.

Now consider the more general formulation with liquidity differences between bonds and equity indexed by an arbitrary $\theta \in[0,1]$. Figure 2 reports the asset returns and equity premia corresponding to various economies indexed by $\theta$ and $\sigma$, with $\theta$ ranging from 0 to $3 \%$, and $\sigma$ ranging from 1 to 5. Specifically, the figure depicts the "level sets" for the theoretical average equity returns, bond returns, and equity premia. The first panel, for instance, shows the various $\theta-\sigma$ combinations that generate a given average equity return. Equity returns are independent of $\theta$, so the level sets are flat lines. For example, if $\sigma$ is slightly above 4 (if $\sigma=4.072$, to

\footnotetext{
${ }^{24}$ For $\sigma$ ranging from 7 to 10 , the magnitude of the $\omega^{e}$ wedge generated by the model is relatively close to some of the mean values one obtains from data sets other than the one used by Kocherlakota (1996). See Table 3 in İmrohoroğlu (2003), for example. There, he compares estimates of the wedges for two measures of the "riskfree" rate, the usual one based on 90-day Treasury bills, and another based on high-grade long-term corporate bonds; and for two measures of equity returns, the standard one based on S\&P 500, and another based on S\&P 500 but subject to the adjustments suggested by McGrattan and Prescott (2001, 2003). Their adjustments are intended to account for taxes, regulatory constraints, and diversification costs. McGrattan and Prescott's emphasis on defining the equity premium relative to long-term bonds - as opposed to relative to 90-day Treasury bills - seems to be an attempt to control for possible liquidity premia: "One problem [with Mehra and Prescott's analysis] is interpreting the return on a 90-day T-bill as the rate at which households intertemporally substitute consumption. We do not interpret it as such. Treasury bills provide considerable liquidity services and are a negligible part of individuals' long-term debt holdings" (McGrattan and Prescott (2003), p. 393).
} 
be precise), the model economy generates an average equity return of $8 \%$ for any value of $\theta$. Similarly, the level sets in the middle panel show the various $\theta-\sigma$ combinations that generate a given average bond return. Notice that even for this relatively narrow parameter range $(0 \leq \theta \leq 0.03$ and $1 \leq \sigma \leq 5)$, the model can display a wide range of bond returns, as high as $5 \%$ and as low as $-10 \%$. For example, if $\sigma=4$, the equilibrium average bond return would be $1 \%$ if $\theta=0.0222$. The last panel shows the average equity premium generated by the model for each $\theta-\sigma$ combination. For a given value of $\sigma$, the equity premium is increasing in $\theta$, and in fact, it is more responsive to $\theta$ for larger values of $\sigma$.
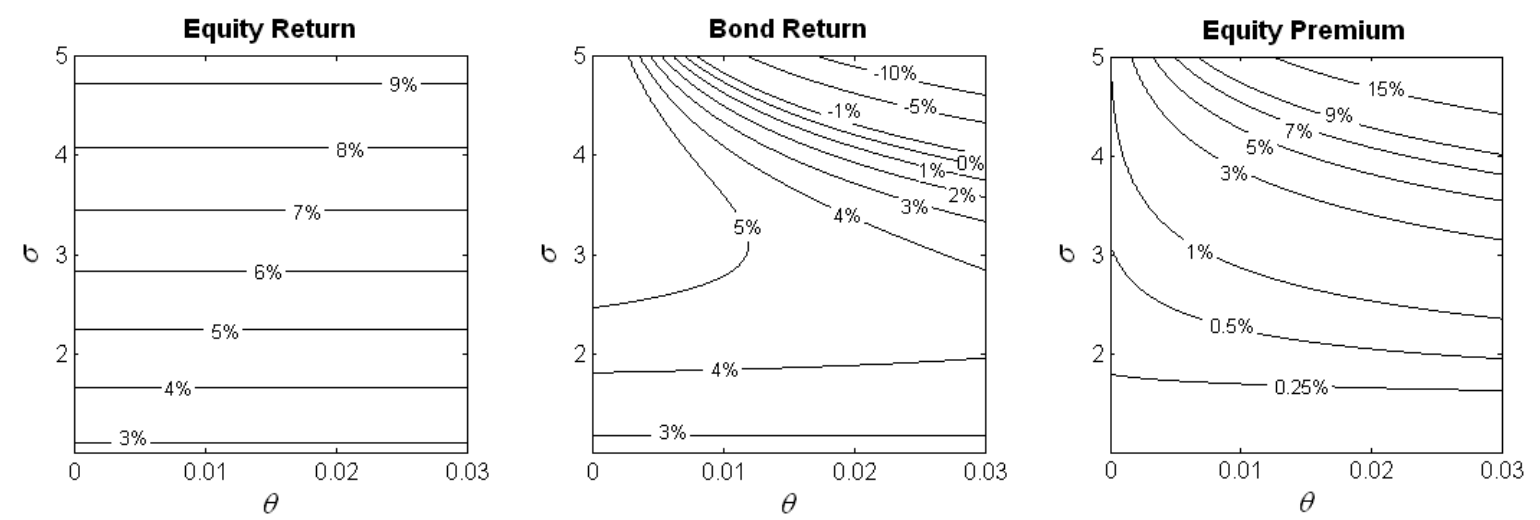

Figure 2: Economies with exogenous liquidity differences

I can now address one of the questions posed in the introduction: for any given view on the value that $\sigma$ should take, how large does $\theta$ (the relative assumed "illiquidity" of equity) have to be for the model to generate an average risk-free rate of $1 \%$ and an average equity premium that matches the long-term average for the U.S. economy? The answer is Table 4.

Table 4 was generated with the baseline parametrization used to generate Table 3 . The difference is that in Table 4, for each value of $\sigma$, the parameter $\theta$ was chosen so that the riskfree rate is equal to $1 \%$, whenever possible. (The middle panel of Figure 2 gives a clear idea of how this can be done.) The resulting value of $\theta$, denoted $\theta^{*}$, is reported in the last column.

The key difference between an economy with $\theta>0$ relative to one with $\theta=0$ is that in the 


\begin{tabular}{cccccc}
\hline \hline$\sigma$ & $\begin{array}{c}\text { Equity } \\
\text { Return }\end{array}$ & $\begin{array}{c}\text { Bond } \\
\text { Return }\end{array}$ & $\begin{array}{c}\text { Equity } \\
\text { Premium }\end{array}$ & $\begin{array}{c}\text { EE Wedges } \\
\omega^{e}, \omega^{b}\end{array}$ & $\theta^{*}$ \\
\hline 1 & 2.83 & 2.70 & 0.13 & 0,0 & - \\
2 & 4.58 & 1 & 3.58 & $.0316,-.0316$ & .3799 \\
3 & 6.27 & 1 & 5.27 & $.0453,-.0453$ & .0742 \\
4 & 7.89 & 1 & 6.89 & $.0576,-.0576$ & .0222 \\
5 & 9.42 & 1 & 8.42 & $.0687,-.0687$ & $.062 \times 10^{-1}$ \\
6 & 10.88 & 1 & 9.88 & $.0786,-.0786$ & $.015 \times 10^{-1}$ \\
7 & 12.24 & 1 & 11.24 & $.0873,-.0873$ & $.032 \times 10^{-2}$ \\
8 & 8.80 & 1 & 7.80 & $.0526,-.0950$ & $3.40 \times 10^{-5}$ \\
9 & 5.97 & 1 & 4.97 & $.0240,-.1016$ & $2.45 \times 10^{-6}$ \\
10 & 4.35 & 1 & 3.35 & $.0062,-.1072$ & $7.26 \times 10^{-8}$ \\
\hline
\end{tabular}

Table 4: Benchmark economy with exogenous liquidity differences

former there are two types of decentralized trades: some where agents can pay using bonds and equity, and a fraction $\theta$ where they can use only bonds. This means that in any given state of the world, agents may face binding liquidity constraints in all decentralized trades, just in those where only bonds can be used as a medium of exchange, or in none of the decentralized trades. Formally, in addition to the set $\Omega(\cdot)$ defined in $(20)$, there is now another set,

$$
\Omega_{\theta}\left(B_{i}\right)=\left\{j \in\{1,2\}: \frac{B_{i}}{\gamma_{j}}<\frac{\kappa^{1-\sigma}}{\sigma-1}\left[1-(1+\epsilon)^{1-\sigma}\right]\right\},
$$

that contains the states for which the liquidity constraints are binding in meetings where only bonds can be used in exchange when the outstanding stock of bonds is $\frac{B_{i}}{\gamma_{j}} d$.

The first row of Table 4 is identical to the first row of Table 3 (and of Table 2). That is, if $\sigma=1$, the agents' liquidity needs are so modest that the value of their equilibrium bond holdings is large enough to allow them to buy the first-best quantity $\epsilon \kappa d_{t}$ in every round of decentralized trade, for all realizations of the dividend process. Formally, $\Omega_{\theta}\left(B_{i}\right)=\varnothing$ for all $i$ and $\Omega\left(B_{i}\right)=\varnothing$, since $\Omega\left(B_{i}\right) \subseteq \Omega_{\theta}\left(B_{i}\right)$. Thus, the equilibrium is independent of the value of $\theta$.

For $\sigma=2$, agents' liquidity needs are already strong enough so that the liquidity constraint is no longer slack in all decentralized meetings. (The nonzero wedges indicate the agent is sometimes short of liquidity.) In fact, for the benchmark parametrization, the equilibrium has $\Omega_{\theta}\left(B_{i}\right)=\{1,2\}$ for all $i$. That is, for all realizations of the aggregate endowment process 
and every outstanding stock of bonds implied by the government's policy rule, the liquidity constraint binds in decentralized trades where the buyer can use only bonds. With $\sigma=2$ liquidity constraints do not bind in decentralized trades where both equity shares and bonds can be used as means of payment, i.e., $\Omega\left(B_{i}\right)=\varnothing$ for all $i$. The equilibrium remains qualitatively the same (in the sense that $\Omega\left(B_{i}\right)=\varnothing$ and $\Omega_{\theta}\left(B_{i}\right)=\{1,2\}$ for all $i$ ) up to $\sigma=\sigma_{h}$. For $\sigma>\sigma_{h}$, the liquidity motive becomes so strong that the equilibrium has $\Omega_{\theta}\left(B_{i}\right)=\Omega\left(B_{i}\right)=\{1,2\}$ for all $i$; that is, liquidity constraints bind at all dates, in all decentralized trades - even when agents can pay with bond and equity shares. ${ }^{25}$

Figure 3 illustrates and complements the findings summarized in Table 4. For each value of $\sigma$ ranging from 1 to 10 , the dashed line gives the corresponding value of $\theta^{*}$, which can be read off the left vertical axis. As mentioned while discussing the first row of Table 4, there is a $\sigma_{m} \approx 1.760$ such that there is no $\theta \leq 1$ that can make the bond return $1 \%$ for $\sigma<\sigma_{m}$. Thus, the first point on the dashed line is $\left(\sigma_{m}, \theta^{*}\right)$ with $\theta^{*}=1$. As we increase $\sigma$ away from $\sigma_{m}$, the implied $\theta^{*}$ falls rapidly for relatively small values of $\sigma$, e.g., up to $\sigma=5$, and levels off-at very low values - for larger $\sigma$.

For each value of $\sigma$, the solid line in Figure 3 displays the equity premium generated by the model with $\theta=\theta^{*}$. (The percentage equity premium can be read off the right vertical axis.) For values of $\sigma$ between 1 and $\sigma_{l} \approx 1.695<\sigma_{m}$, the liquidity needs are so mild that the bond portfolio alone is enough to satisfy them. This is the range at which $\Omega\left(B_{i}\right)=\Omega_{\theta}\left(B_{i}\right)=\varnothing$, so neither bonds nor equity yields a liquidity return. The equilibrium is independent of $\theta$, and the equity premium increases slightly with $\sigma$ just as in Mehra-Prescott. The first kink (the one at $\sigma_{l}$ ) occurs because for $\sigma>\sigma_{l}$, the liquidity constraint starts to bind in restricted matches. However, even though bonds yield a liquidity return, it is too small to lower the measured return to $1 \%$, even if $\theta=1$. That is, even with $\theta=1$, the measured bond return is above $1 \%$ for $\sigma<\sigma_{m}$. The second kink (the one at $\sigma=\sigma_{m}$ ) indicates that from then on (i.e., to

\footnotetext{
${ }^{25}$ Again, notice that the column that reports the Euler equation "wedges" $\omega^{e}$ and $\omega^{b}$ can be used as an indicator of the binding patterns of the liquidity constraints. To see this, refer back to (18) and (19) and note that: ( $i$ ) $\omega^{e}=\omega^{b}=0$ if $\Omega_{\theta}\left(B_{i}\right)=\Omega\left(B_{i}\right)=\varnothing,(i i) \omega^{b}<0$ iff $\Omega_{\theta}\left(B_{i}\right) \neq \varnothing$, and $(i i i) \omega^{e}=\left|\omega^{b}\right|$ iff $\Omega\left(B_{i}\right)=\varnothing$.
} 


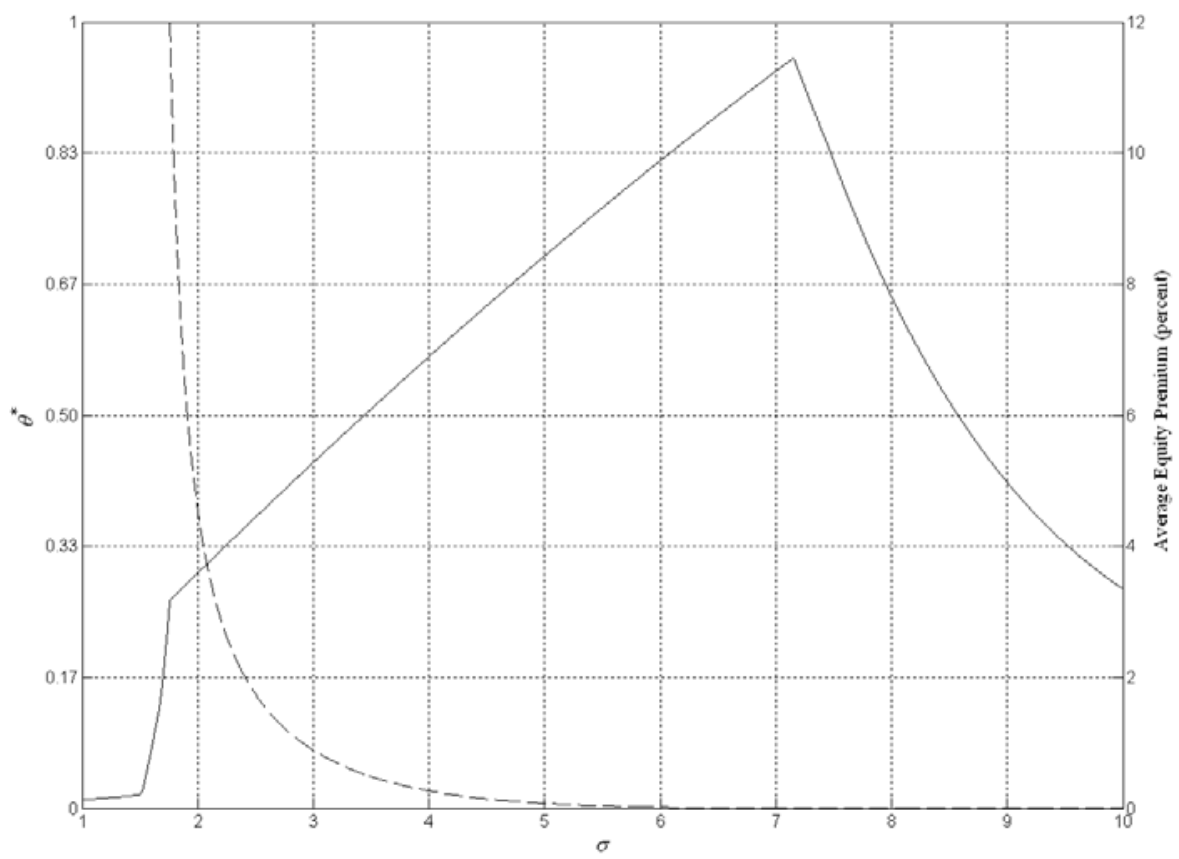

Figure 3: The equity premium in economies with liquidity differences

the right of $\left.\sigma_{m}\right), \theta$ can and is chosen to peg the bond return at 1\%. For values of $\sigma$ between $\sigma_{m}$ and $\sigma_{h}$, the implied equilibrium has $\Omega_{\theta}\left(B_{i}\right)=\{1,2\}$ and $\Omega\left(B_{i}\right)=\varnothing$ for all $i$. The equity premium peaks at $11.45 \%$, corresponding to an economy with $\sigma=\sigma_{h}$ and $\theta^{*} \approx 4.21 \times 10^{-5}$. The third kink (the one at $\sigma_{h}$ ) occurs because for parametrizations with $\sigma$ larger than $\sigma_{h}$, liquidity needs are so severe that agents are constrained in all trades, restricted and unrestricted, i.e., $\Omega\left(B_{i}\right)=\Omega_{\theta}\left(B_{i}\right)=\{1,2\}$ for all $i$.

Notice that equity returns are identical across Tables 3 and 4 , as is to be expected from the results in the first panel of Figure 2. Thus, just as for the economy with $\theta=0$, equity returns coincide with those of the Mehra-Prescott economy for $\sigma \leq \sigma_{h}$. Although equity shares can in principle provide liquidity in the economies of Tables 3 and 4, they don't for relatively low levels of $\sigma$. Accordingly, for this range of $\sigma$, equity returns are increasing in $\sigma{ }^{26}$ Interestingly,

\footnotetext{
${ }^{26}$ Just as in Table 3, equity returns start falling for $\sigma$ above $\sigma_{h}$, once equity shares can be used to relax liquidity constraints at the margin, i.e., once liquidity constraints start binding in decentralized trades where agents can
} 
the equilibrium equity returns and the equity premium implied by $\sigma=3, \sigma=4$, and $\sigma=5$ in Table 4 lie within the range of estimates reported in Table $1 .{ }^{27}$ The corresponding values of $\theta^{*}$ are $0.0742,0.0222$, and 0.0062 , respectively. So, for example, if $\sigma=4$, this says that in 2 out of 100 decentralized trading opportunities, agents find themselves in a situation in which they cannot trade away the shares in their portfolios. At face value, this proportion seems rather small, especially given that the institutional arrangements for trading away equity and bonds are rather different in actual economies. ${ }^{28}$ But this view may leave room for dissent, so in the following section, I propose a more systematic way to gauge the absolute size of the difference in the relative ability of shares and bonds to serve as mediums of exchange, which are needed for the liquidity motive to be able to rationalize the full observed equity premium. ${ }^{29}$

The signs and magnitudes of the Euler equation wedges in Table 4 are in line with those estimated by Kocherlakota (1996) using Mehra and Prescott's (1985) data. For example, if $\sigma=5,\left(\omega^{e}, \omega^{b}\right)=(.0687,-.0687)$, compared to $(.0433,-.0675)$ in Kocherlakota's Tables 2 and 3. For $\sigma=4$, the model implies $\left(\omega^{e}, \omega^{b}\right)=(.0576,-.0576)$, and Kocherlakota's estimates are $(.0464,-.0569)$, and for $\sigma=3,\left(\omega^{e}, \omega^{b}\right)=(.0453,-.0453)$, compared to $(.0496,-.0448) .{ }^{30}$

To see the effects that liquidity differences among assets have on equilibrium returns, consider Figure 4, which is analogous to Figure 1, but with $\theta=0.0222$ (the $\theta^{*}$ corresponding to $\sigma=4$ in Table 4). The only difference between the first panel of Figure 1 and the first panel of

pay with any combination of shares and bonds.

${ }^{27}$ Note that although the equity return (and consequently also the equity premium) is too high for $\sigma=6$ and 7 , the equity return and the equity premium are also in line with the data for $\sigma=8$. Liquidity constraints bind in all trades for $\sigma \geq \sigma_{h}$, so in this range equity yields a liquidity return, and this depresses the measured intrinsic return back to a level consistent with some of the estimates in Table 1.

${ }^{28}$ Here I have in mind the fact that from an investor's standpoint, executing and settling a sale of shares routinely takes longer than executing and settling a sale of bonds (see footnote 8). These differences may imply that once in a while, the proceeds of a sale of equity shares may not be available in time to take advantage of a fleeting trade opportunity.

${ }^{29}$ Lest there be any confusion, I want to stress that these exercises are not meant to suggest that only liquidity considerations are behind the observed equity premium. The spirit is rather to put this liquidity mechanism to an extreme test by asking whether it could conceivably account for the whole premium. To the extent that these sorts of liquidity considerations are only one of several ingredients of a broader theory of the equity premium, the implied values of $\theta^{*}$ will be even smaller.

${ }^{30}$ For $\sigma=8$, the other parametrization that can generate reasonable average equity return and premium, the model has $\left(\omega^{e}, \omega^{b}\right)=(.0526,-.0950)$, and Kocherlakota reports $(.0341,-.0910)$. 
Figure 4 is the dashed line, i.e., the measured bond return, which is much lower in the latter. The most noteworthy feature of the second panel of Figure 4 is the sheer size of the equity premium in an economy with only modest liquidity differences. (Note that the vertical axis here goes from 0 to $7 \%$ but only up to $3 \%$ in the second panel of Figure 1.) For example, the equity premium is $7 \%$ if $\sigma \approx 4.0125$.
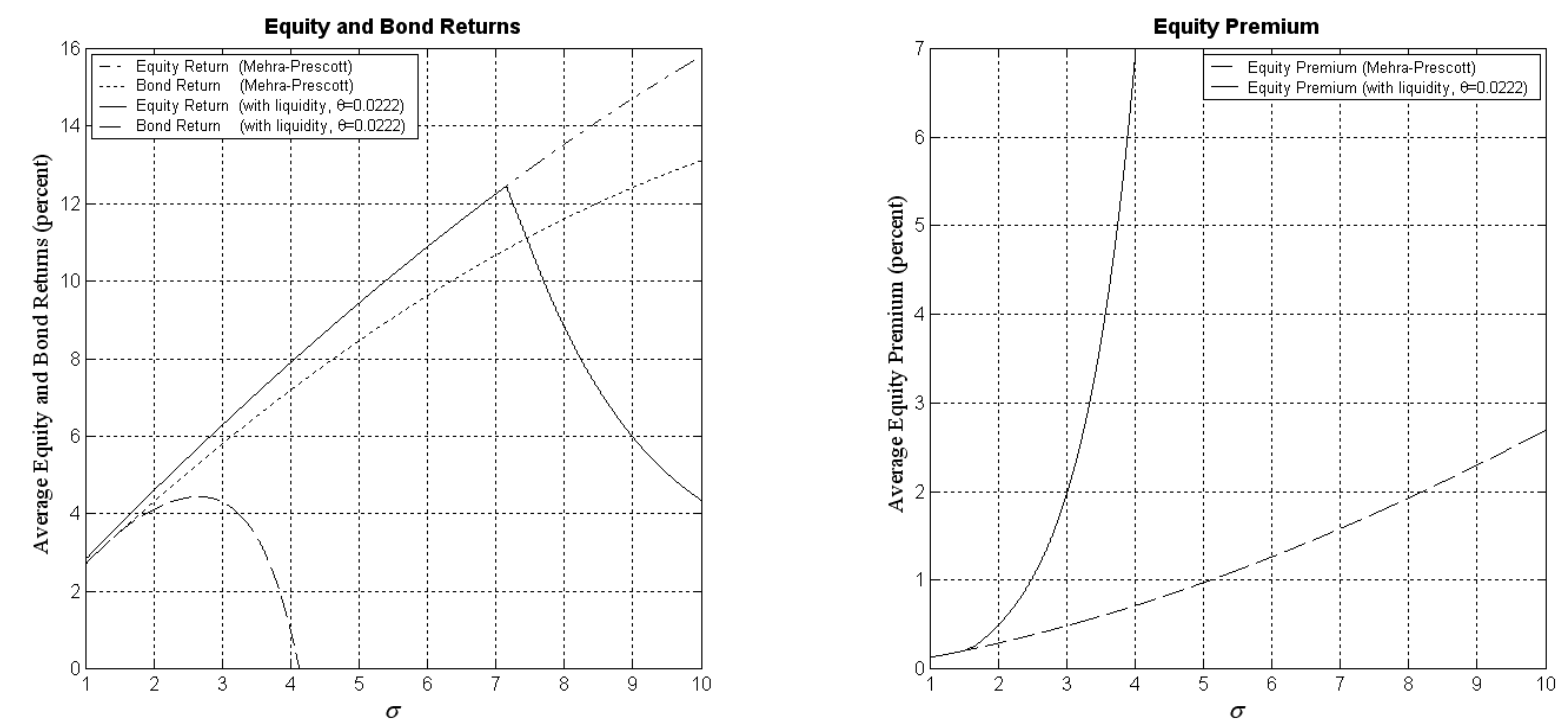

Figure 4: Returns for Mehra-Prescott and for the economy with liquidity $(\theta=0.0222)$

The focus of the paper is on the first moments of asset returns, but I conclude this section with a remark on second moments. The standard deviations of equity returns in the model with liquidity (for any value of $\theta$ ) do not differ much from the Mehra-Prescott model. For example, they are $3.6 \%$ for $\sigma=1,6.2 \%$ for $\sigma=5$, and $9.6 \%$ for $\sigma=10$. (The standard deviations are identical to the Mehra-Prescott counterparts up to $\sigma=\sigma_{h}$ and vary only slightly for higher values of $\sigma$.) Even though these standard deviations are rather low (the standard deviation is roughly $16 \%$ in the data), the Mehra-Prescott model still generates a Sharpe ratio that is too low ( 0.035 for $\sigma=1,0.156$ for $\sigma=5$, and 0.276 for $\sigma=10$ ) relative to the standard estimates (about 0.5). Interestingly, the Sharpe ratio is too high in the model with liquidity 
for the parametrizations that generate the right premium. Of course, this is just another way of saying that the liquidity mechanism, as modeled here, does not induce additional volatility in equity returns.

\subsection{Bond-equity trade-volume bounds}

A natural way to assess whether the values of $\theta$ that are needed for the model to rationalize the equity premium as a liquidity premium are "reasonable" follows from the realization that $\theta$ determines the frequency with which bonds get traded vis-à-vis shares. For example, in the extreme case with $\theta=1$, equity shares never change hands in this economy: each agent holds a single equity share at the end of the period, and since these shares are never used in decentralized trades, each agent also enters every second subperiod with the same share, so no shares are traded along the equilibrium. Conversely, in this case the volume of bonds traded will be positive along the equilibrium path, since at least some bonds will be exchanged in the first subperiod and again in the second subperiod. The idea, then, is to construct the ratio of the value of bonds traded in a given period (as a proportion of the total value of outstanding stock of bonds), relative to the value of shares traded (as a proportion of the total value of outstanding equity).

Let $v_{i j}^{b}$ and $v_{i j}^{s}$ denote the quantities or volumes of bonds and equity shares that are traded in a period when the aggregate state is $(d, j)$ and the outstanding stock of bonds is $\frac{B_{i}}{\gamma_{j}} d$. Then $v_{i j}^{b}$ and $\phi_{j}^{s} d v_{i j}^{s}$ are, respectively, the value of bonds and shares that change hands during the period, expressed in terms of fruit, the numeraire. The total values of outstanding bonds and

shares in the same period are $\frac{B_{i}}{\gamma_{j}} d$ and $\phi_{j}^{s} d$. The proportion of the outstanding value of the stock of bonds traded during the period - the bond turnover ratio-is $v_{i j}^{b} \gamma_{j} /\left(B_{i} d\right)$. Similarly, the proportion of the outstanding value of equity that was traded during the period - the equity turnover ratio - is $v_{i j}^{s}$. Therefore,

$$
v_{i j}=\frac{v_{i j}^{b} \gamma_{j}}{v_{i j}^{s} B_{i} d}
$$

can be used as a measure of the turnover of bonds relative to the turnover of equity. 
The total volumes traded are $v_{i j}^{b}=\frac{\hat{\alpha}}{2}\left[\theta p_{i j}^{b}+(1-\theta) \hat{p}_{i j}^{b}\right]$, and $v_{i j}^{s}=\hat{\alpha}(1-\theta) \hat{p}_{i j}^{s}$, where $p_{i j}^{b}$ is the quantity of bonds traded in a match where shares cannot be used, and $\left(\hat{p}_{i j}^{s}, \hat{p}_{i j}^{b}\right)$ is the portfolio that changes hands in a match where both shares and bonds can be used for payment, in a period when the aggregate state is $(d, j)$ and the outstanding stock of bonds is $\frac{B_{i}}{\gamma_{j}} d$. (Clearly, as mentioned earlier, $v_{i j}^{s} \rightarrow 0$ as $\theta \rightarrow 1$, so values of $\theta$ close to 1 are unreasonable in that they would imply an unrealistically large volume of trade for bonds relative to shares.)

To get a sense for how the quantities $p_{i j}^{b}, \hat{p}_{i j}^{s}$, and $\hat{p}_{i j}^{b}$ of assets traded in the decentralized market are determined, it is convenient to consider the four possible types of double-coincidence trades a buyer may find himself in when $\theta \in(0,1)$. The buyer may be in a meeting where he can use shares as means of payment, or where he cannot, and in each case, the relevant liquidity constraint may be binding or slack. In the two cases where the relevant constraint is binding, the agent simply spends all his portfolio if the meeting is one in which both can be used in exchange, or just all his bond holdings if the meeting is one in which shares cannot be traded. If the liquidity constraint is slack in a meeting where only bonds can be used in exchange (for example, as is the case when $\sigma=1$ ), he will spend just enough bonds to afford $\epsilon \kappa d$. However, if the liquidity constraint is slack in trades where either asset can be used as a medium of exchange, then only the real value of the portfolio that changes hands is pinned down by the equilibrium - the precise composition of the portfolio that the buyer gives the seller in exchange for the $\epsilon \kappa d$ quantity of fruit is indeterminate. ${ }^{31}$

This means that in those cases where the liquidity constraint is slack in trades where either asset can be used in exchange (as is the case for $\sigma=2$ through 7 in the benchmark parametrization of the model with $\theta \in(0,1))$, the precise trading volumes of bonds and shares are indeterminate in the equilibrium. Nonetheless, for each value of $\theta$, it is possible to derive implied upper and lower bounds for $v_{i j}$, denoted $\bar{v}_{i j}$ and $\underline{v}_{i j}$, respectively. From these, I derive the average (with respect to the model's invariant distribution) upper and lower bounds, $\bar{v}$ and $\underline{v}$ for the ratio of the value of traded bonds (as a proportion of the value of the outstanding stock

\footnotetext{
${ }^{31}$ For details, see the discussion around (27) in the appendix (Section A.3).
} 
of bonds) to the value of traded shares (as a proportion of the value of outstanding equity). (See the appendix for details.) Values of $\theta$ that imply bounds $\bar{v}$ and $\underline{v}$ such that the ratio of bond to equity trade volumes computed from actual data is smaller than $\underline{v}$ would be deemed too large. Similarly, values of $\theta$ for which the value of the ratio observed in the data is larger than the implied $\bar{v}$ would be too low.

From Table 4, the values of $\sigma$ for which the model is able to generate a risk-free rate of $1 \%$ and an equity premium that is in line with the data are $\sigma=3,4$, and 5. For $\sigma=5, \theta=0.0062$, and this value of $\theta$ implies $[\underline{v}, \bar{v}]=[.01,2.55]$. This means that the model is consistent with the value of traded bonds (relative to the value of outstanding bonds) being at most two and a half times and at least $1 \%$ of the value of traded equity (relative to the value of outstanding equity). Similarly, the volume bounds implied by $\theta=0.0222$ (the value corresponding to $\sigma=4$ ) are $[.09,6.29]$, and those implied by $\theta=0.0742$ (the value corresponding to $\sigma=3$ ) are $[0.7,20]$. The bound for the pair $(\sigma, \theta)=(6, .0015)$ is [.0015, 1.17]. Naturally, $\bar{v}$ is increasing in $\theta$ (decreasing in $\sigma)$.

\section{Discussion}

The bounds of Hansen and Jagannathan (1991) provide a way of assessing the magnitude of asset-pricing puzzles and are often used as diagnostics tests for asset-pricing models. I begin by asking how the Mehra-Prescott economy and the benchmark economy with liquidity fare against these bounds for different values of $\sigma$. This is a useful way to understand how the two models differ at the core, i.e., in terms of the first and second moments of their respective stochastic discount factors.

Let $m$ denote a stochastic discount factor that satisfies $E(m R)=\mathbf{1}$, the unconditional version of the agent's Euler equations, where $R=\left[R_{1}, R_{2}\right]^{\prime}$ and $\mathbf{1}$ is a vector of ones. Let $\Sigma_{m}$ denote the standard deviation of $m$. The cup-shaped line in Figure 5 is the Hansen-Jagannathan bound for returns; i.e., $\Sigma_{m} \geq\left[b^{\prime} \operatorname{cov}(R, R) b\right]^{1 / 2}$, with $b=[\operatorname{cov}(R, R)]^{-1}[\mathbf{1}-E(m) E(R)]$. The

straight line is the Hansen-Jagannathan bound on excess returns; i.e., $\Sigma_{m} \geq\left[b^{\prime} \operatorname{cov}(z, z) b\right]^{1 / 2}$, 
with $b=-[\operatorname{cov}(z, z)]^{-1} E(m) E(z)$ and $z=R_{1}-R_{2} \cdot{ }^{32}$ The lines are drawn for the original Mehra-Prescott data: $R^{\prime}=[1.07,1.01], \operatorname{var}\left(R_{1}\right)=0.0274, \operatorname{var}\left(R_{2}\right)=0.00308$, and $\operatorname{cov}\left(R_{1}, R_{2}\right)=0.00104$. According to these data, the minimum standard deviation an admissible stochastic discount factor must have is about 0.3509 (the minimum height of the cup-shaped curve). This value of $\Sigma_{m}$ corresponds to an $E(m)$ of about 0.9855 . Since the sides of the cup are rather steep, a model that satisfies this bound must have a stochastic discount factor with a mean that is close to 0.9855 , unless one is willing to accept a dramatically higher standard deviation for the stochastic discount factor. (If agents have access to a risk-free return, then this value of $E(m)$ implies a risk-free rate close to 1.0147.)

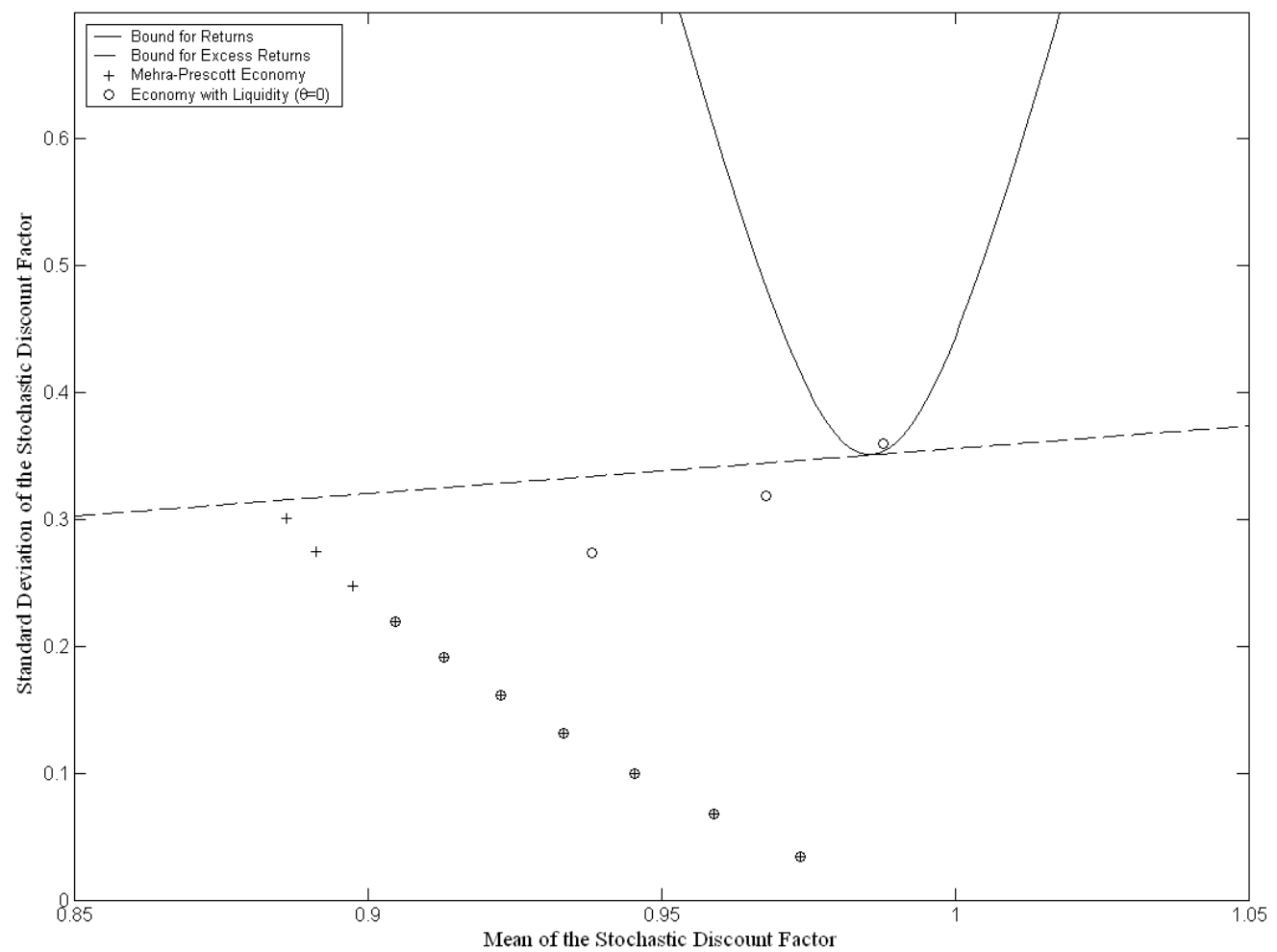

Figure 5: Hansen-Jagannathan bounds

\footnotetext{
${ }^{32}$ See Ljungqvist and Sargent (2000) for a textbook treatment. The bounds in Figure 1 are identical to those in Ljungqvist and Sargent's Figure 10.4, p. 287. McGrattan and Prescott (2003) suggest some adjustments to the basic data that result in a different-lower-pair of bounds. See İmrohoroğlu (2003) for details.
} 
Consider the basic model with no exogenous liquidity differences between assets. The Euler equations (14) and (15) imply: $\sum_{j} \mu_{i j} m_{i j} \hat{R}_{i j}^{s}=\sum_{j} \mu_{i j} m_{i j} \hat{R}_{i}^{b}=1$, where $L_{i j}=L_{i j}^{s}$ (as given in (16), with $\theta=0$ ) and $m_{i j}=\beta \gamma_{j}^{-\sigma} L_{i j}$ is the stochastic discount factor between states $i$ and $j$. The circles in Figure 5 give the mean-standard deviation pairs of this discount factor implied by the benchmark calibration of Table 3, for values of $\sigma$ ranging from 1 to 10 . The crosses are the analogous mean-standard deviation pairs obtained by setting $L_{i j}=1$ for all $i$ and $j$. In this case, the stochastic discount factor for state $j$ is just $m_{j}=\beta \gamma_{j}^{-\sigma}$ : this is the basic Mehra-Prescott economy of Table 2. For example, if $\sigma=1$, the mean-standard deviation pair is $(0.9737,0.0346)$ for both models. This is the lowest point in the figure; higher points correspond to higher values of $\sigma$. In fact, for $\sigma=1$ through 7 , the liquidity constraints are slack (recall that the Euler equation wedges are zero in Table 3), so both models share the same stochastic discount factor, and hence they generate the same mean-standard deviation pair for each $\sigma$. (These seven points appear marked with a circle and a cross in Figure 5.) The stochastic discount factors of the two models diverge for $\sigma=8$ and higher, i.e., once the liquidity constraints start to bind. The liquidity mechanism increases the standard deviation, but especially the mean of the stochastic discount factor. For instance, at $\sigma=10$, the model with liquidity has a stochastic discount factor with mean and standard deviation $(0.9878,0.3602)$ just inside the admissible cup-shaped area. ${ }^{33}$

I would like to stress that this discussion is not intended to suggest that the model developed here solves the equity premium puzzle because the point corresponding to $\sigma=10$ lies inside the cup-shaped area. Instead, the spirit is that, by understanding how the new ingredients I have added to the standard Lucas-type asset-pricing model (e.g., decentralized exchange, anonymity, and the resulting need for a medium of exchange) affect the moments of the stochastic discount factor that prices returns, one can get a better sense for why these ingredients can enhance the ability of the model to explain asset returns.

\footnotetext{
${ }^{33}$ For $\sigma=10$, the Mehra-Prescott economy implies $(0.8861,0.3010)$, which does not even satisfy the bound for excess returns. It takes a value of $\sigma$ of about 20 in the Mehra-Prescott model to bring the mean and standard deviation of their stochastic discount factor within the bounds implied by their data.
} 
Next, consider the more general formulation with $\theta \in[0,1]$. The (unconditional versions of the) corresponding Euler equations (2) and (3) imply the following expression for the average risk premium:

$$
\begin{aligned}
E\left(\hat{R}^{s}-\hat{R}^{b}\right)= & \frac{-\operatorname{cov}\left(R^{s}, M R S\right)}{E(M R S)}+\frac{\operatorname{cov}\left(R^{b}, M R S\right)}{E(M R S)}-\operatorname{cov}\left(\hat{R}^{s}, L^{s}\right)+\operatorname{cov}\left(\hat{R}^{b}, L^{b}\right) \\
& +\left[E\left(L^{b}\right)-1\right] E\left(\hat{R}^{b}\right)-\left[E\left(L^{s}\right)-1\right] E\left(\hat{R}^{s}\right),
\end{aligned}
$$

where $M R S_{t+1}$ denotes the marginal rate of substitution, $\beta U^{\prime}\left(c_{t+1}\right) / U^{\prime}\left(c_{t}\right)$. (Recall that $\hat{R}_{t+1}^{i}$ is the measured return of asset $i$, and $R_{t+1}^{i}=L_{t+1}^{i} \hat{R}_{t+1}^{i}$ is the full return, as perceived by the agents in the model.) The first two terms are standard: excess returns are partly due to the fact that the full return on equity covaries with the growth rate of consumption more than does the average bond return. The third term is an adjustment for the degree to which equity returns covary with the liquidity constraints; i.e., it reflects the extent to which equity shares are a good hedge against binding liquidity constraints. For example, if the liquidity constraints that can be relaxed with shares are looser in periods when the equity return is relatively high, then $\operatorname{cov}\left(\hat{R}^{s}, L^{s}\right)<0$, and the third term tends to magnify the measured equity premium. The fourth term is an analogous adjustment for bonds. The last two terms reflect the liquidity return differential between the assets: their sum will be positive if $\ln E\left(L^{b}\right)-\ln E\left(L^{s}\right)>$ $\ln E\left(\hat{R}^{s}\right)-\ln E\left(\hat{R}^{b}\right)$, namely, if the average (geometric) excess liquidity return of bonds over shares is larger than the average (geometric) excess measured return of shares over bonds. When this is the case, the combination of the last two terms adds on to the equity premium as it is typically measured. ${ }^{34}$

To get some intuition about the signs and relative magnitudes of the various components of the equity premium, Tables 5 and 6 report the first and second moments of the asset returns,

\footnotetext{
${ }^{34}$ If $L_{t}^{s}=L_{t}^{b}=1$ with probability 1 at all $t$, as is the case for the benchmark parametrization with $\theta=0$ for $\sigma=7$ or lower, and for any parametrization of the Mehra-Prescott model, then

$$
E\left(R^{s}\right)-R^{b}=\frac{-\operatorname{cov}\left(R^{s}, M R S\right)+\operatorname{cov}\left(R^{b}, M R S\right)}{E(M R S)},
$$
}

and the model relies only on the first two terms discussed above to account for the whole equity premium. 


\begin{tabular}{r|l|ccccccc}
\hline \hline & \multirow{2}{*}{ Mean } & \multicolumn{7}{|c}{ Variance-Covariance } \\
\cline { 3 - 8 } & & $\hat{R}^{s}$ & $\hat{R}^{b}$ & $L^{s}$ & $L^{b}$ & $R^{s}$ & $R^{b}$ & $M R S$ \\
\hline$\hat{R}^{s}$ & 1.043518 & .00914 & .00282 & -.00268 & -.00268 & .00728 & .00039 & -.02569 \\
$\hat{R}^{b}$ & 1.015041 & & .00269 & -.00023 & -.00023 & .00287 & .00274 & -.00219 \\
$L^{s}$ & 1.103996 & & & .00099 & .00099 & -.00193 & .00075 & .00946 \\
$L^{b}$ & 1.103996 & & & & .00099 & -.00193 & .00075 & .00946 \\
$R^{s}$ & 1.149354 & & & & & .00601 & .00121 & -.01849 \\
$R^{b}$ & 1.120370 & & & & & & .00378 & .00719 \\
$M R S$ & 0.886144 & & & & & & & .09063 \\
\hline
\end{tabular}

Table 5: Moments of returns, baseline with $\sigma=10$ and $\theta=0$

\begin{tabular}{r|l|ccccccc}
\hline \hline & \multirow{2}{*}{ Mean } & \multicolumn{7}{|c}{ Variance-Covariance } \\
\cline { 3 - 8 } & & $\hat{R}^{s}$ & $\hat{R}^{b}$ & $L^{s}$ & $L^{b}$ & $R^{s}$ & $R^{b}$ & $M R S$ \\
\hline$\hat{R}^{s}$ & 1.078871 & .00305 & .00042 & 0 & .00028 & .00305 & .00074 & -.00701 \\
$\hat{R}^{b}$ & 1.01 & & .00039 & 0 & $-2.8 \times 10^{-8}$ & .00042 & .00042 & -.00036 \\
$L^{s}$ & 1.00 & & & 0 & 0 & 0 & 0 & 0 \\
$L^{b}$ & 1.061942 & & & & .00003 & .00028 & .00003 & -.00073 \\
$R^{s}$ & 1.078871 & & & & & .00305 & .00074 & -.00701 \\
$R^{b}$ & 1.072561 & & & & & & .00048 & -.00112 \\
$M R S$ & 0.933394 & & & & & & & .01722 \\
\hline
\end{tabular}

Table 6: Moments of returns, baseline with $\sigma=4$ and $\theta=0.0222$

liquidity premia, and marginal rate of substitution implied by the model. Table 5 corresponds to the baseline economy, but with $\theta=0$ and $\sigma=10$ (the tenth line of Table 3). In this case, we know that there is no liquidity differential, i.e., $L^{b}=L^{s}=L$ always, so the premium can be written as

$$
\underbrace{E\left(\hat{R}^{s}-\hat{R}^{b}\right)}_{.028477}=\underbrace{\frac{-\operatorname{cov}\left(R^{s}, M R S\right)}{E(M R S) E(L)}}_{.018904}+\underbrace{\frac{\operatorname{cov}\left(R^{b}, M R S\right)}{E(M R S) E(L)}-\frac{\operatorname{cov}\left(\hat{R}^{s}, L\right)}{E(L)}}_{.00735}+\underbrace{\frac{\operatorname{cov}\left(\hat{R}^{b}, L\right)}{E(L)}}_{.002433}
$$

The numbers below each term are for the decomposition implied by the model. The first two terms account for about $92 \%$ of the premium and the third for $8.5 \%$. In this case, since $\operatorname{cov}(L, M R S)>0$, the liquidity mechanism is causing the total return on equity, $R^{s}$, to covary more with the MRS than the measured return $\hat{R}^{s}$, and this tends to bring the equity premium down from what it would have been in an economy with $L=1 .^{35}$ However, the fact that

\footnotetext{
${ }^{35}$ For Mehra-Prescott, in this parametrization the premium is 0.02689 , and it is accounted for fully by the
} 
$\operatorname{cov}(L, M R S)>0$ turns $\operatorname{cov}\left(R^{b}, M R S\right)$ positive even though $\operatorname{cov}\left(\hat{R}^{b}, M R S\right)<0$ (see Table 5$)$. Intuitively, the measured asset returns $\hat{R}^{s}$ and $\hat{R}^{b}$ tend to be high in times when the growth rate of consumption is high (the MRS low), but these are also times when the value of the average portfolio is high, which means that each agent will face looser liquidity constraints, or equivalently, that $L$ will tend to be low in those periods.

Table 6 corresponds to the baseline economy, with $\sigma=4$ and $\theta=0.0222$ (the fourth row of Table 4). In this case $L^{s}=1$ always, so the third and fifth terms of (22) are identically zero, and the premium can be decomposed as

$$
\underbrace{E\left(\hat{R}^{s}-\hat{R}^{b}\right)}_{.068871} \approx \underbrace{\left[-\operatorname{cov}\left(R^{s}, M R S\right)+\operatorname{cov}\left(R^{b}, M R S\right)\right] E(M R S)^{-1}}_{.0063}+\underbrace{\left[E\left(L^{b}\right)-1\right] E\left(\hat{R}^{b}\right)}_{.062571} .
$$

The liquidity differential between bonds and equity accounts for about $90 \%$ of the equity premium. ${ }^{36}$ In yet another way to try to assess which fraction of the equity premium is due to risk and which to liquidity considerations, I have set $\delta$ (the standard deviation of the growth rate of the endowment) to zero in the baseline. For example, with $\sigma=4$ and $\theta=0.0222$ (see Tables 4 and 6 ), the equity premium goes down from $6.8871 \%$ to $6.3146 \%$. So in this case, about $90 \%$ of the equity premium is due to the fact that equity pays an illiquidity premium.

\section{Conclusion}

I have presented an asset-pricing model in which financial assets are valued for their liquiditythe degree to which they are valued as a medium of exchange - as well as for being claims to streams of consumption. The key implications of the model for average asset returns, the equity-premium and risk-free rate puzzles, were explored analytically and quantitatively.

Explicitly modeling the exchange process, and allowing for the possibility that the value of equity shares and bonds may partly depend on the role that each plays in exchange, rationalizes the two most commonly addressed asset-pricing anomalies. Quantitatively, the model performs negative covariance of equity returns with the MRS.

\footnotetext{
${ }^{36}$ The fourth term in $(22), \operatorname{cov}\left(\hat{R}^{b}, L^{b}\right)$, is negative, but zero to the fifth decimal, as a percentage.
} 
better than the Mehra-Prescott frictionless benchmark, even if shares are just as useful as bonds for exchange purposes. But with standard constant relative risk aversion preferences, it still takes a coefficient of relative risk aversion of about 10 for the model to be consistent with asset return data.

If, in addition, one allows for the fact that bonds may be (slightly) better suited than equity shares to play the medium-of-exchange role, then the model is able to match the historical average return to equity and the risk-free rate for the United States with values of the risk aversion coefficient between 3 and 5. These results indicate that prying deeper into the microeconomics of the decentralized exchange process may add to our understanding of how asset prices are determined in actual economies.

Kocherlakota (1996) ended his survey on the equity premium puzzle by drawing a parallel between the pure theory of money-much of which seeks to understand issues such as the coexistence of interest-bearing risk-free nominal bonds and fiat money - and the branch of financial economics that deals with the equity-premium puzzle. He argued that "we must seek to identify what fundamental features of goods and asset markets lead to large risk-adjusted price differences between stocks and bonds." And he concluded with, "While I have no idea what these 'fundamental features' are, it is my belief that any true resolution to the equity premium puzzle lies in finding them."

In this paper, I have tried to pursue this line of reasoning a step further. I have advanced some candidate "fundamental features." These features are those that go into making an asset a medium of exchange, which - aside from the measured properties of the asset - are bound to be related to the frequency of trade, the determination of the terms of trade, and the nature of the information structure. I have also asked whether these features stand a chance quantitatively. They do.

Having identified these features, at a deeper level, the key issue becomes, why is asset $X$ more generally accepted or better suited than asset $Y$ to function as a medium of exchange? In terms of the equity premium, the next step is to explain precisely how these particular features 
can lead to differences in acceptability or, more generally, in the readiness for exchange between equity and bonds. In this regard, Kocherlakota (2003) and Zhu and Wallace (2005) may provide some valuable hints. 


\section{References}

[1] Aiyagari, S. Rao, and Mark Gertler. "Asset Returns with Transaction Costs and Uninsured Individual Risk." Journal of Monetary Economics 27(3) (June 1991): 311-331.

[2] Aiyagari, S. Rao, and Neil Wallace. "Government Transaction Policy, the Medium of Exchange, and Welfare." Journal of Economic Theory 74(1) (May 1997): 1-18.

[3] Aiyagari, S. Rao, Neil Wallace, and Randall Wright. "Coexistence of Money and Interestbearing Securities." Journal of Monetary Economics 37(3) (June 1996): 397-419.

[4] Bansal, Ravi, and John Wilbur Coleman II. "A Monetary Explanation of the Equity Premium, Term Premium, and Risk-free Rate Puzzles." Journal of Political Economy 104(6) (December 1996): 1135-1171.

[5] Diamond, Peter A. "Aggregate Demand Management in Search Equilibrium." Journal of Political Economy 90(5) (October 1982): 881-894.

[6] Duffie, Darrell, Nicolae Gârleanu, and Lasse Heje Pedersen. "Over-the-Counter Markets." Econometrica 73(6) (November 2005a): 1815-1847.

[7] Greenwood, Jeremy, Richard Rogerson, and Randall Wright. "Household Production in Real Business Cycle Theory." In Frontiers of Business Cycle Research, ed. Thomas J. Cooley. Princeton, NJ: Princeton University Press, 1995.

[8] Hansen, Lars Peter, and Ravi Jagannathan. "Implications of Security Market Data for Models of Dynamic Economies." Journal of Political Economy 99(2) (April 1991): 225262.

[9] Hansen, Lars Peter, and Kenneth J. Singleton. "Generalized Instrumental Variables Estimation of Nonlinear Rational Expectations Models." Econometrica 50(5) (September 1982): 1269-1286. 
[10] He, Hua, and David M. Modest. "Market Frictions and Consumption-Based Asset Pricing." Journal of Political Economy 103(1) (February 1995): 94-117.

[11] Heaton, John, and Deborah J. Lucas. "The Importance of Investor Heterogeneity and Financial Market Imperfections for the Behavior of Asset Prices." Carnegie-Rochester Conference Series on Public Policy 42 (June 1995): 1-32.

[12] Heaton, John, and Deborah J. Lucas. "Evaluating the Effects of Incomplete Markets on Risk Sharing and Asset Pricing." Journal of Political Economy 104(3) (June 1996): 443487.

[13] Ibbotson Associates. Stocks, Bonds, Bills, and Inflation: 2000 Yearbook. Chicago: Ibbotson Associates, 2001.

[14] İmrohoroğlu, Selahattin. "A Note on the McGrattan and Prescott (2003) Adjustments and the Equity Premium Puzzle.” University of Southern California mimeo, 2003.

[15] Kiyotaki, Nobuhiro, and John Moore. "Liquidity and Asset Prices." International Economic Review 46(2) (May 2005): 317-350.

[16] Kiyotaki, Nobuhiro, and Randall Wright. "On Money as a Medium of Exchange." Journal of Political Economy 97(4) (August 1989): 927-954.

[17] Kocherlakota, Narayana. "The Equity Premium: It's Still a Puzzle." Journal of Economic Literature 34(1) (March 1996): 42-71.

[18] Kocherlakota, Narayana R. "Societal Benefits of Illiquid Bonds." Journal of Economic Theory 108(2) (February 2003): 179-193.

[19] Krueger, Dirk, and Fabrizio Perri. "Does Income Inequality Lead to Consumption Inequality? Evidence and Theory." Federal Reserve Bank of Minneapolis mimeo, 2005. 
[20] Lagos, Ricardo. "Asset Prices, Liquidity, and Monetary Policy." Federal Reserve Bank of Minneapolis mimeo (in progress), 2006.

[21] Lagos, Ricardo, and Guillaume Rocheteau. "Money and Capital as Competing Media of Exchange." Federal Reserve Bank of Minneapolis Staff Report 341, August 2004.

[22] Lagos, Ricardo, and Randall Wright. "A Unified Framework for Monetary Theory and Policy Analysis." Journal of Political Economy 113(3) (June 2005): 463-484.

[23] Li, Yiting, and Randall Wright. "Government Transaction Policy, Media of Exchange, and Prices." Journal of Economic Theory 81(2) (August 1998): 290-313.

[24] Ljungqvist, Lars, and Thomas J. Sargent. Recursive Macroeconomic Theory. Cambridge, MA: MIT Press, 2000.

[25] Lucas, Deborah J. "Asset Pricing with Undiversifiable Income Risk and Short Sales Constraints: Deepening the Equity Premium Puzzle." Journal of Monetary Economics 34(3) (December 1994): 325-341.

[26] Lucas, Robert E., Jr. "Asset Prices in an Exchange Economy." Econometrica 46(6) (November 1978): 1426-1445.

[27] Luttmer, Erzo G. J. "Asset Pricing in Economies with Frictions." Econometrica 64(6) (November 1996): 1439-1467.

[28] McGrattan, Ellen R., and Edward C. Prescott. "Taxes, Regulations, and Asset Prices." Federal Reserve Bank of Minneapolis Working Paper 610, 2001.

[29] McGrattan, Ellen R., and Edward C. Prescott. "Average Debt and Equity Returns: Puzzling?" American Economic Review 93(2) (May 2003): 392-397.

[30] Mehra, Rajnish, and Edward C. Prescott. "The Equity Premium: A Puzzle." Journal of Monetary Economics 15(2) (March 1985): 145-161. 
[31] Mehra, Rajnish, and Edward C. Prescott. "The Equity Premium in Retrospect." In Handbook of the Economics of Finance, ed. G. M. Constantinides, M. Harris, and R. M. Stulz. Amsterdam: Elsevier/North-Holland, 2003.

[32] Neumeyer, Pablo A., and Fabrizio Perri. "Business Cycles in Emerging Economies: The Role of Interest Rates." Journal of Monetary Economics 52(2) (March 2005): 345-380.

[33] Ravikumar, B., and Enchuan Shao. "Search Frictions and Asset Price Volatility." University of Iowa mimeo, 2005.

[34] Reed, Robert, and Christopher Waller. "Money and Risk Sharing." University of Kentucky and University of Notre Dame mimeo, 2005.

[35] Shi, Shouyong. "A Divisible Search Model of Fiat Money." Econometrica 65(1) (January 1997): $75-102$.

[36] Shi, Shouyong. "Nominal Bonds and Interest Rates." International Economic Review 46(2) (May 2005): 579-612.

[37] Shiller, Robert J. Market Volatility. Cambridge, MA: MIT Press, 1990.

[38] Siegel, Jeremy J. Stocks for the Long Run. 2nd ed. New York: Irwin, 1998.

[39] Telmer, Chris, I. "Asset-pricing Puzzles and Incomplete Markets." Journal of Finance 48(5) (December 1993): 1803-1832.

[40] Vayanos, Dimitri, and Tan Wang. "Search and Endogenous Concentration of Liquidity in Asset Markets." London School of Economics mimeo, 2005.

[41] Vayanos, Dimitri, and Pierre-Olivier Weill. "A Search-Based Theory of the On-the-Run Phenomenon.” NYU Stern School of Business mimeo, 2005.

[42] Wallace, Neil. "A Dictum for Monetary Theory." Federal Reserve Bank of Minneapolis Quarterly Review 22(1) (Winter 1998): 20-26. 
[43] Weill, Pierre-Olivier. "Leaning Against the Wind." NYU Stern School of Business mimeo, 2005a.

[44] Weill, Pierre-Olivier. "Liquidity Premia in Dynamic Bargaining Markets." NYU Stern School of Business mimeo, 2005b.

[45] Zhu, Tao, and Neil Wallace. "Pairwise Trade and Coexistence of Money and Higher-Return Assets." Pennsylvania State University mimeo, 2005. 


\section{A Appendix}

\section{A.1 Euler equations}

Here I show how to derive the Euler equations (2) and (3). First, note that the bargaining solution derived at the end of Section 2 , and the fact that $W\left[\mathbf{a}+\mathbf{p}^{i}(\mathbf{a}, \tilde{\mathbf{a}}), \mathbf{s}\right]=W(\mathbf{a}, \mathbf{s})+$ $\lambda \mathbf{p}^{i}(\mathbf{a}, \tilde{\mathbf{a}})$, imply that the expected value of search can be written as

$$
V(\mathbf{a}, \mathbf{s})=\alpha \sum_{i=1,2} \theta_{i} \mathcal{S}\left(\boldsymbol{\lambda}^{i} \mathbf{a} ; d\right)+W(\mathbf{a}, \mathbf{s})+(1-2 \alpha) \bar{u}(d)
$$

where $\mathcal{S}\left(\boldsymbol{\lambda}_{t}^{i} \mathbf{a}_{t} ; d_{t}\right)=u\left[(1-\epsilon) \kappa d_{t}+q\left(\boldsymbol{\lambda}_{t}^{i} \mathbf{a}_{t} ; \kappa d_{t}\right)\right]+u\left[(1+\epsilon) \kappa d_{t}-q\left(\boldsymbol{\lambda}_{t}^{i} \mathbf{a}_{t} ; \kappa d_{t}\right)\right]$. Then, focus on the agent's maximization problem in the centralized market, i.e., (1). The optimal choices of $c_{t}$ and $n_{t}$ satisfy

$$
\begin{aligned}
U^{\prime}\left(c_{t}\right) & =\frac{A_{t}}{w_{t}} \\
v^{\prime}\left(Z_{t} n_{t}\right) & =\frac{A_{t}}{Z_{t}} .
\end{aligned}
$$

The portfolio choice, $\mathbf{a}_{t+1}$, satisfies

$$
U^{\prime}\left(c_{t}\right) \phi_{t}^{i}=\beta E_{t} \frac{\partial V\left(\mathbf{a}_{t+1}, \mathbf{s}_{t+1}\right)}{\partial a_{t+1}^{i}}
$$

for $i=b, s$, where

$$
\begin{aligned}
\frac{\partial V\left(\mathbf{a}_{t+1}, \mathbf{s}_{t+1}\right)}{\partial a_{t+1}^{b}} & =\left[1+\alpha \sum_{i=1,2} \theta_{i}\left(\frac{u^{\prime}\left[(1-\epsilon) \kappa d_{t+1}+q\left(\boldsymbol{\lambda}_{t+1}^{i} \mathbf{a}_{t+1} ; \kappa d_{t+1}\right)\right]}{u^{\prime}\left[(1+\epsilon) \kappa d_{t+1}-q\left(\boldsymbol{\lambda}_{t+1}^{i} \mathbf{a}_{t+1} ; \kappa d_{t+1}\right)\right]}-1\right)\right] \lambda_{t+1}^{b}, \\
\frac{\partial V\left(\mathbf{a}_{t+1}, \mathbf{s}_{t+1}\right)}{\partial a_{t+1}^{s}} & =\left[1+\alpha \theta_{2}\left(\frac{u^{\prime}\left[(1-\epsilon) \kappa d_{t+1}+q\left(\lambda_{t+1}^{2} \mathbf{a}_{t+1} ; \kappa d_{t+1}\right)\right]}{u^{\prime}\left[(1+\epsilon) \kappa d_{t+1}-q\left(\lambda_{t+1}^{2} \mathbf{a}_{t+1} ; \kappa d_{t+1}\right)\right]}-1\right)\right] \lambda_{t+1}^{s},
\end{aligned}
$$

are obtained by differentiating (23). Using $\lambda_{t}^{1}=\left(\lambda_{t}^{b}, 0\right)$ and $\lambda_{t}^{2}=\left(\lambda_{t}^{b}, \lambda_{t}^{s}\right)$, with $\lambda_{t}^{b}=\frac{A_{t}}{w_{t}}$ and $\lambda_{t}^{s}=\left(\phi_{t}^{s}+d_{t}\right) \lambda_{t}^{b},(26)$ can be rewritten as $(2)-(5)$.

\section{A.2 Equilibrium allocations and prices}

In this section, I derive the full set of equilibrium allocations and prices for the economy of Section 4. Given that $U(c)=u(c)=\frac{c^{1-\sigma}}{1-\sigma}$ and that the supply of bonds is set according to 
the rule $B_{t+1}=f\left(d_{t}, x_{t}\right)=B_{i} d_{t}$ if $x_{t}=\gamma_{i}$, and focusing on recursive equilibria where share prices are homogeneous of degree one in $d$, leads to (14)-(17). The equilibrium asset prices are $\phi^{s}(d, i)=\phi_{i}^{s} d$ and $\phi^{b}(d, i)=\phi_{i}^{b}$, where $\left(\phi_{i}^{s}, \phi_{i}^{b}\right)_{i=1}^{n}$ solve (14) and (15) for $i=1, \ldots, n$. The decision rules for bond holdings, shares, and consumption are $a^{b}(d, i)=B_{i} d, a^{s}(d, i)=1$ and $c(d, i)=d$, respectively.

A buyer who enters a bilateral meeting holding $\mathbf{a}=\left(a^{s}, a^{b}\right)$ in a period where the state is $(d, i)$ has a portfolio that is worth $\left(d+\phi_{i}^{s} d\right) a^{s}+a^{b}$ in terms of fruit in the round of centralized trade that follows. ${ }^{37}$ Such a buyer purchases

$$
q\left(a^{b} d^{-\sigma} ; \kappa d\right)=\min \left\{\epsilon \kappa,(1+\epsilon) \kappa-\left(\frac{1}{[(1+\epsilon) \kappa]^{1-\sigma}+(\sigma-1) \frac{a^{b}}{d}}\right)^{\frac{1}{\sigma-1}}\right\} d
$$

in a decentralized meeting in which he can pay only with bonds, or

$$
q\left[\left(d+\phi_{i}^{s} d\right) a^{s} d^{-\sigma}+a^{b} d^{-\sigma}, \kappa d\right]=\min \left\{\epsilon \kappa,(1+\epsilon) \kappa-\left(\frac{1}{[(1+\epsilon) \kappa]^{1-\sigma}+(\sigma-1)\left[\left(1+\phi_{i}^{s}\right) a^{s}+\frac{a^{b}}{d}\right]}\right)^{\frac{1}{\sigma-1}}\right\} d
$$

in a meeting in which he can pay with bonds or shares. Along the equilibrium path, if the state today is $\left(d_{t}, i\right)$, all agents bring $a^{s}\left(d_{t}, i\right)=1$ and $a^{b}\left(d_{t}, i\right)=B_{i} d_{t}$ into the following period's round of decentralized trade. Note that if in the following period the state is $\left(d_{t+1}, j\right)$, then the bond holdings of agents in that period, $a^{b}\left(d_{t}, i\right)$, can be written as $\frac{B_{i}}{\gamma_{j}} d_{t+1}$. Thus, along the equilibrium path, in a period where the state is $(d, j)$ and the outstanding stock of bonds is $\frac{B_{i}}{\gamma_{j}} d$, a buyer purchases a quantity

$$
\min \left\{\epsilon \kappa,(1+\epsilon) \kappa-\left(\frac{1}{[(1+\epsilon) \kappa]^{1-\sigma}+(\sigma-1) \frac{B_{i}}{\gamma_{j}}}\right)^{\frac{1}{\sigma-1}}\right\} d
$$

in a decentralized meeting in which he can pay only with bonds, or a quantity

$$
\min \left\{\epsilon \kappa,(1+\epsilon) \kappa-\left(\frac{1}{[(1+\epsilon) \kappa]^{1-\sigma}+(\sigma-1)\left(1+\phi_{j}^{s}+\frac{B_{i}}{\gamma_{j}}\right)}\right)^{\frac{1}{\sigma-1}}\right\} d
$$

\footnotetext{
${ }^{37}$ In terms of marginal utility, the value of this portfolio is $\boldsymbol{\lambda}(d, i) \mathbf{a}$, where $\boldsymbol{\lambda}(d, i)=\left[\lambda^{s}(d, i), \lambda^{b}(d)\right]$, with $\lambda^{b}(d)=U^{\prime}(d)$, and $\lambda^{s}(d, i)=U^{\prime}(d)\left(1+\phi_{i}^{s}\right) d$. Intuitively, $\lambda^{b}(d, i)$ and $\lambda^{s}(d, i)$ are the end-of-period values (in terms of marginal utility of apples) of a bond and a share, respectively, in a period where the state is $(d, i)$.
} 
in a meeting in which he can pay with bonds or shares. In the former, the buyer pays

$$
\min \left\{\frac{B_{i}}{\gamma_{j}},\left[(1+\epsilon)^{1-\sigma}-1\right] \frac{\kappa^{1-\sigma}}{1-\sigma}\right\} d
$$

bonds, and in the latter, he pays with a combination of bonds and shares, $\mathbf{p}^{2}=\left(p^{s}, p^{b}\right) \in \mathbb{R}$, with real value (in terms of apples)

$$
\left(1+\phi_{j}^{s}\right) p^{s} d+p^{b}=\min \left\{1+\phi_{j}^{s}+\frac{B_{i}}{\gamma_{j}},\left[(1+\epsilon)^{1-\sigma}-1\right] \frac{\kappa^{1-\sigma}}{1-\sigma}\right\} d,
$$

where $0 \leq p^{s} \leq 1$ and $0 \leq p^{b} \leq \frac{B_{i}}{\gamma_{j}} d$. The real value of the portfolio that changes hands is pinned down, but the precise composition is indeterminate. ${ }^{38}$

To derive equilibrium real wage $\left(w_{t}\right)$ and the remaining individual choices, namely, labor demand $\left(n_{t}\right)$, consumption of general goods in the second subperiod $\left(y_{t}\right)$, and labor supply $\left(h_{t}\right)$, we need to parametrize $v(\cdot)$ and $A_{t}$. Let $v(y)=\frac{y^{1-\sigma}}{1-\sigma}, A_{t}=A Z_{t}^{1-\sigma}$, where $\sigma$ and $A$ are positive constants, and recall that $Z_{t}=d_{t} \cdot{ }^{39}$ Then, (24) and (25) imply $w(d)=A d, n(d)=n^{*}$, and $y(d)=n^{*} d$, where $n^{*}=A^{-1 / \sigma}$.

Finally, along the equilibrium path, an agent who enters the centralized market with portfolio $\left(a_{t}^{b}, a_{t}^{s}\right)$ at the end of period $t$ chooses to work

$$
h_{t}=n^{*}+\frac{1}{w_{t}}\left[\left(\phi_{t}^{s}+d_{t}\right)\left(1-a_{t}^{s}\right)+B_{t}-a_{t}^{b}\right] .
$$

\footnotetext{
${ }^{38}$ This is also a feature that can be found in Lagos and Rocheteau (2004), a version of Lagos and Wright (2005) where capital goods and fiat money compete as media of exchange.

${ }^{39}$ Setting $A_{t}=A Z_{t}^{1-\sigma}$ means that the disutility from work depends on the level of technology of the economy. This specification is useful for two reasons. First, it makes the agent's preferences consistent with balanced growth. And second, it allows one to derive relatively simple conditions to ensure that the constraints $0 \leq h_{t} \leq \bar{n}$ are slack at all dates and for all states of the world - which must be the case for the distribution of asset holdings to be degenerate in the equilibrium. Alternatively, one could assume $A_{t}=A \bar{\gamma}^{(1-\sigma) t}$, where $\bar{\gamma}$ is the mean growth rate of the economy, as done in many RBC models (see Greenwood, Rogerson, and Wright (1995), or Neumeyer and Perri (2005)). This formulation is enough to guarantee that $h_{t}$ has no secular trend, but in the present context, one would still have to verify that the structure of the shocks and other primitives of the model are such that the choice of $h_{t}$ is interior for all realizations of the uncertainty. In fact, this should not be too difficult, for instance, if the variance of the shocks is small, since both formulations coincide if the economy grows deterministically. To conclude this technical digression, I would like to stress that although the particular specification for $A_{t}$ (and $v$ ) matters "globally," i.e., to ensure that the equilibrium is indeed within the class of those with a degenerate distribution of asset holdings - as was conjectured for the derivations - it does not matter "locally," e.g., for the behavior of asset prices. That is, given that the equilibrium distribution of asset holdings is degenerate, asset prices are independent of $A_{t}$ and $v$. Observe that (14)-(17) were derived before parametrizing $A_{t}$ and $v$.
} 
Intuitively, agents supply labor to finance their consumption of the general good (the first term) and possibly also to rebalance their portfolios. ${ }^{40}$

Recall that the analysis has been predicated on the premise that the distribution of assets is degenerate at the beginning of each decentralized round of trade - which will indeed be the case, as in Lagos and Wright (2005) - provided the constraints $0 \leq h_{t} \leq \bar{n}$ are always slack. Next, I provide two alternative sets of sufficient conditions on parameters, such that this is indeed the case along the equilibrium path. In a period where the state is $(d, i)$ and the outstanding stock of bonds is $\frac{B_{j}}{\gamma_{i}} d$, the right-hand side of $(28)$ can be written as

$$
h\left(B_{j}, i, d\right)=A^{-1 / \sigma}+\frac{1}{A}\left[\left(1+\phi_{i}^{s}\right)\left(1-a^{s}\right)+\frac{B_{j}}{\gamma_{i}}-\frac{a^{b}}{d}\right] .
$$

Along the conjectured equilibrium path, $0 \leq a^{s} \leq 2$ and $0 \leq a^{b} \leq 2 \frac{B_{j}}{\gamma_{i}} d$. That is, after a round of decentralized trade, an agent can enter the centralized market neither with a negative asset position nor holding more than twice his beginning-of-period portfolio. Thus, the inequalities $\underline{h}(j, i) \leq h\left(B_{j}, i, d\right) \leq \bar{h}(j, i)$, where $\underline{h}(j, i) \equiv A^{-1 / \sigma}-\frac{1}{A}\left(1+\phi_{i}^{s}+\frac{B_{j}}{\gamma_{i}}\right)$, and $\bar{h}(j, i) \equiv A^{-1 / \sigma}+$ $\frac{1}{A}\left(1+\phi_{i}^{s}+\frac{B_{j}}{\gamma_{i}}\right)$, hold along the equilibrium path in a period where the dividend level is $d$, the realization of the dividend growth rate is $\gamma_{i}$, and the outstanding stock of bonds is $\frac{B_{j}}{\gamma_{i}} d$. Let $\Delta=\max _{i, j}\left(1+\phi_{i}^{s}+\frac{B_{j}}{\gamma_{i}}\right)$, and note that $\Delta$ is independent of $A$. Then, it follows that the inequalities $A^{-1 / \sigma}-\frac{\Delta}{A} \leq h\left(B_{j}, i, d\right) \leq A^{-1 / \sigma}+\frac{\Delta}{A}$ hold along the equilibrium path in every period and all states (i.e., for every $d, B_{j}$ and $i$ ). Thus, $\Delta \leq A^{\frac{\sigma-1}{\sigma}} \leq A \bar{n}-\Delta$ implies $0 \leq h\left(B_{j}, i, d\right) \leq \bar{n}$, for all $i, d$ and $B_{j}$, and is a sufficient condition for the constraints $0 \leq h_{t} \leq \bar{n}$ to be slack at all dates, with probability one.

An alternative, simpler way to ensure that $h_{t}$ stays off corners is to interpret it as effort and proceed as follows. Since the asset-pricing implications of the model are independent of the specification of preferences over general goods, $v(y)$, one can normalize $v(y)=y$. Then,

\footnotetext{
${ }^{40}$ For example, an agent who neither bought nor sold in the decentralized market enters the centralized market holding $\left(a_{t}^{b}, a_{t}^{s}\right)=\left(B_{t}, 1\right)$ and therefore chooses $h_{t}=n^{*}$.
} 
the problem in the centralized market becomes

$$
\begin{gathered}
W\left(\mathbf{a}_{t}, \mathbf{s}_{t}\right)=\max _{c_{t}, n_{t}, h_{t}, \mathbf{a}_{t+1}}\left\{U\left(c_{t}\right)+Z_{t} n_{t}-A_{t} h_{t}+\beta E V\left(\mathbf{a}_{t+1}, \mathbf{s}_{t+1}\right)\right\} \\
\text { s.t. } c_{t}+w_{t} n_{t}+\phi_{t} \mathbf{a}_{t+1}=\left(\phi_{t}^{s}+d_{t}\right) a_{t}^{s}+a_{t}^{b}+w_{t} h_{t}-\tau_{t} .
\end{gathered}
$$

Interpreting $h_{t}$ as effort amounts to assuming that $\bar{n}$ is arbitrarily large, and then the maximization is subject only to $0 \leq n_{t}$ and $0 \leq h_{t}$ (the nonnegativity constraints on $c_{t}$ and $\mathbf{a}_{t+1}$ will not bind). Assume, as above, that the disutility from work is indexed by the state of technology; in particular, let $A_{t}=Z_{t}$. Then, the budget constraint implies $h_{t}-n_{t}=$ $\frac{1}{w_{t}}\left\{c_{t}+\phi_{t} \mathbf{a}_{t+1}+\tau_{t}-\left[\left(\phi_{t}^{s}+d_{t}\right) a_{t}^{s}+a_{t}^{b}\right]\right\}$. This can be substituted into the objective to yield the unconstrained maximization problem:

$$
W\left(\mathbf{a}_{t}, \mathbf{s}_{t}\right)=\boldsymbol{\lambda}_{t} \mathbf{a}_{t}-\frac{A_{t}}{w_{t}} \tau_{t}+\max _{c_{t}}\left[U\left(c_{t}\right)-\frac{A_{t}}{w_{t}} c_{t}\right]+\max _{\mathbf{a}_{t+1}}\left[-\frac{A_{t}}{w_{t}} \boldsymbol{\phi}_{t} \mathbf{a}_{t+1}+\beta E V\left(\mathbf{a}_{t+1}, \mathbf{s}_{t+1}\right)\right]
$$

Since $h_{t}=\frac{1}{w_{t}}\left\{c_{t}+\phi_{t} \mathbf{a}_{t+1}+\tau_{t}-\left[\left(\phi_{t}^{s}+d_{t}\right) a_{t}^{s}+a_{t}^{b}\right]\right\}+n_{t}$, and $n_{t}$ can be chosen to be any arbitrary nonnegative number, it follows that the constraint $h_{t} \geq 0$ can be made slack at all dates and states.

\section{A.3 Bond-equity trade-volume bounds}

In this section I derive the bond-equity trade-volume bounds discussed in Section 4.3. In equilibrium, in a period where the state is $(d, j)$ and the outstanding stock of bonds is $\frac{B_{i}}{\gamma_{j}} d$, the portfolio that is traded in meetings where only bonds can be used to pay is $\mathbf{p}_{i j}^{1}=\left(0, p_{i j}^{b}\right)$, with $p_{i j}^{b}=\min \left\{\frac{B_{i}}{\gamma_{j}},\left[(1+\epsilon)^{1-\sigma}-1\right] \frac{\kappa^{1-\sigma}}{1-\sigma}\right\} d$. In meetings where both assets can be used in exchange, the portfolio that is traded is $\mathbf{p}_{i j}^{2}=\left(1, \frac{B_{i}}{\gamma_{j}} d\right)$ if the liquidity constraint binds and any vector $(0,0) \leq\left(p_{i j}^{s}, p_{i j}^{b}\right) \leq\left(1, \frac{B_{i}}{\gamma_{j}} d\right)$ that satisfies $(27)$ if it is slack. Given all this, it is possible to derive an upper and a lower bound for the quantities of bonds and shares traded in each state, $v_{i j}^{b}$ and $v_{i j}^{s}$. To derive the upper bound, resolve the (potential) indeterminacy in $\left(\hat{p}_{i j}^{s}, \hat{p}_{i j}^{b}\right)$, namely, in the quantities of shares and bonds that get traded in a meeting where either can be used for payment, by assuming that buyers always follow a spend-bonds-first rule. This rule 
means that $\left(\hat{p}_{i j}^{s}, \hat{p}_{i j}^{b}\right)=\left(\underline{p}_{i j}^{s}, \bar{p}_{i j}^{b}\right)$, where

$$
\begin{aligned}
& \bar{p}_{i j}^{b}=\min \left\{\frac{B_{i}}{\gamma_{j}},\left[(1+\epsilon)^{1-\sigma}-1\right] \frac{\kappa^{1-\sigma}}{1-\sigma}\right\} d \\
& \underline{p}_{i j}^{s}=\min \left\{1, \max \left\{\left[(1+\epsilon)^{1-\sigma}-1\right] \frac{\kappa^{1-\sigma}}{1-\sigma}-\frac{B_{i}}{\gamma_{j}}, 0\right\} \frac{1}{1+\phi_{j}^{s}}\right\} .
\end{aligned}
$$

To derive the lower bound, resolve the indeterminacy in $\left(\hat{p}_{i j}^{s}, \hat{p}_{i j}^{b}\right)$ by assuming that buyers follow a spend-shares-first rule. This implies $\left(\hat{p}_{i j}^{s}, \hat{p}_{i j}^{b}\right)=\left(\bar{p}_{i j}^{s}, \underline{p}_{i j}^{b}\right)$, where

$$
\begin{aligned}
& \bar{p}_{i j}^{s}=\min \left\{1,\left[(1+\epsilon)^{1-\sigma}-1\right] \frac{\kappa^{1-\sigma}}{1-\sigma} \frac{1}{1+\phi_{j}^{s}}\right\} \\
& \underline{p}_{i j}^{b}=\min \left\{\frac{B_{i}}{\gamma_{j}}, \max \left\{\left[(1+\epsilon)^{1-\sigma}-1\right] \frac{\kappa^{1-\sigma}}{1-\sigma}-\left(1+\phi_{j}^{s}\right), 0\right\}\right\} d .
\end{aligned}
$$

Thus, $\bar{p}_{i j}^{b}$ and $\underline{p}_{i j}^{s}$ are the quantities of assets traded in matches of type 2 when the buyer uses the spend-bonds-first rule, and $\bar{p}_{i j}^{s}$ and $\underline{p}_{i j}^{b}$ are the assets traded in matches of type 2 when the buyer uses the spend-shares-first rule. (Note that the formulas for $\bar{p}_{i j}^{b}, \underline{p}_{i j}^{s}, \underline{p}_{i j}^{b}$, and $\bar{p}_{i j}^{s}$ are general in that they apply both when the liquidity constraint binds and when it is slack.) Recall that the total quantities traded in the period are $v_{i j}^{b}=\frac{\hat{\alpha}}{2}\left[\theta p_{i j}^{b}+(1-\theta) \hat{p}_{i j}^{b}\right]$ and $v_{i j}^{s}=\hat{\alpha}(1-\theta) \hat{p}_{i j}^{s}$, and define the following bounds on the total quantities traded:

$$
\begin{aligned}
\bar{v}_{i j}^{b} & =\frac{\hat{\alpha}}{2}\left[\theta p_{i j}^{b}+(1-\theta) \bar{p}_{i j}^{b}\right] \\
\underline{v}_{i j}^{b} & =\frac{\hat{\alpha}}{2}\left[\theta p_{i j}^{b}+(1-\theta) \underline{p}_{i j}^{b}\right] \\
\bar{v}_{i j}^{s} & =\hat{\alpha}(1-\theta) \bar{p}_{i j}^{s} \\
\underline{v}_{i j}^{s} & =\hat{\alpha}(1-\theta) \underline{p}_{i j}^{s} .
\end{aligned}
$$

So $\bar{v}_{i j}^{b}$ and $\underline{v}_{i j}^{s}$ are the total quantities of bonds and shares traded under the spend-bonds-first regime, and $\underline{v}_{i j}^{b}$ and $\bar{v}_{i j}^{s}$ are the total quantities of bonds and shares traded under the spendshares-first regime. In turn, these bounds can be used to derive upper and lower bounds for the ratio $v_{i j}$, namely,

$$
\bar{v}_{i j}=\frac{\gamma_{j} \bar{v}_{i j}^{b}}{\underline{v}_{i j}^{s} B_{i} d} \quad \text { and } \quad \underline{v}_{i j}=\frac{\gamma_{j} \underline{v}_{i j}^{b}}{\bar{v}_{i j}^{s} B_{i} d}
$$


Finally, the average upper and lower bounds for the ratio of the value of traded bonds (as a proportion of the value of the outstanding stock of bonds) to the value of traded shares (as a proportion of the value of outstanding equity) are $\bar{v}=\sum_{i} \sum_{j} \bar{\mu}_{i} \mu_{i j} \bar{v}_{i j}$, and $\underline{v}=\sum_{i} \sum_{j} \bar{\mu}_{i} \mu_{i j} \underline{v}_{i j}$. These bounds can be compared with actual trade volume data to assess the plausibility of the underlying value of $\theta$.

\section{A.4 Robustness}

In this section I verify the robustness of the benchmark results to changes in the values of the parameters $\hat{B}, \kappa$, and $\varepsilon$, the key parameters that were not in Mehra and Prescott (1985).

The benchmark parametrization is perhaps too conservative, in the sense that it biases the case against the liquidity mechanism by using 0.3 as the target ratio of $\mathrm{M}^{*}$ to annual GDP, which implied $\hat{B}=0.75$. Tables 7 and 8 are analogous to Tables 3 and 4 , but with $\hat{B}=0.5$, which is consistent with a ratio of $\mathrm{M}^{*}$ to annual GDP of 0.2 (this is roughly the average for the 1975-1995 period). All the other parameters are kept at their benchmark levels. All entries corresponding to $\sigma=1$ through 7 in Table 7 are identical to those in Table 3 . In both, the liquidity constraints bind for $\sigma \geq 8$. When the constraints bind, the asset returns are only slightly lower in Table 7, as is natural, since the relative scarcity of outside bonds means that both assets yield larger liquidity returns. The equity premium is essentially unchanged.

The first four columns of Table 8 are identical to the corresponding columns of Table 4 through $\sigma=7$. Again, equity returns and premia are slightly lower in the economy with lower $\hat{B}$ for higher values of $\sigma$. Since the bond to GDP ratio is smaller in this economy, the liquidity constraint is even more binding in trades where only bonds can circulate, and therefore the implied values for $\theta$ are uniformly lower in Table 8 relative to Table 4 . In Table 8 , the volume bounds implied by the values of $\theta$ corresponding to $\sigma=5,4$, and 3, i.e., those that imply returns consistent with the data, are $[.004,2],[.03,5]$, and $[.23,14]$, respectively.

In the benchmark calibration, I set $\kappa=0.5$ so that the model implies that the fraction of GDP that corresponds to trees that have outstanding tradeable equity shares equals 0.4 . Next, 


\begin{tabular}{ccccc}
\hline \hline$\sigma$ & $\begin{array}{c}\text { Equity } \\
\text { Return }\end{array}$ & $\begin{array}{c}\text { Bond } \\
\text { Return }\end{array}$ & $\begin{array}{c}\text { Equity } \\
\text { Premium }\end{array}$ & $\begin{array}{c}\text { EE Wedges } \\
\omega^{e}, \omega^{b}\end{array}$ \\
\hline 1 & 2.83 & 2.70 & 0.13 & 0,0 \\
2 & 4.58 & 4.30 & 0.28 & 0,0 \\
3 & 6.27 & 5.79 & 0.48 & 0,0 \\
4 & 7.89 & 7.18 & 0.71 & 0,0 \\
5 & 9.42 & 8.45 & 0.97 & 0,0 \\
6 & 10.88 & 9.62 & 1.26 & 0,0 \\
7 & 12.24 & 10.67 & 1.57 & 0,0 \\
8 & 8.70 & 6.65 & 2.05 & $.0010,-.0444$ \\
9 & 5.94 & 3.50 & 2.44 & $.0014,-.0794$ \\
10 & 4.34 & 1.49 & 2.85 & $.0017,-.1028$ \\
\hline
\end{tabular}

Table 7: Robustness, lower $\hat{B}$ with $\theta=0$

\begin{tabular}{cccccc}
\hline \hline$\sigma$ & $\begin{array}{c}\text { Equity } \\
\text { Return }\end{array}$ & $\begin{array}{c}\text { Bond } \\
\text { Return }\end{array}$ & $\begin{array}{c}\text { Equity } \\
\text { Premium }\end{array}$ & $\begin{array}{c}\text { EE Wedges } \\
\omega^{e}, \omega^{b}\end{array}$ & $\theta^{*}$ \\
\hline 1 & 2.83 & 2.70 & 0.13 & 0,0 & - \\
2 & 4.58 & 1 & 3.58 & $.0316,-.0316$ & .078000 \\
3 & 6.27 & 1 & 5.27 & $.0453,-.0453$ & .026300 \\
4 & 7.89 & 1 & 6.89 & $.0576,-.0576$ & .008020 \\
5 & 9.42 & 1 & 8.42 & $.0687,-.0687$ & .002080 \\
6 & 10.88 & 1 & 9.88 & $.0786,-.0786$ & .000464 \\
7 & 12.24 & 1 & 11.24 & $.0873,-.0873$ & $9.0 \times 10^{-5}$ \\
8 & 8.70 & 1 & 7.70 & $.0517,-.0950$ & $8.7 \times 10^{-6}$ \\
9 & 5.94 & 1 & 4.94 & $.0237,-.1016$ & $5.8 \times 10^{-7}$ \\
10 & 4.34 & 1 & 3.34 & $.0061,-.1072$ & $1.6 \times 10^{-8}$ \\
\hline
\end{tabular}

Table 8: Robustness, lower $\hat{B}$ with exogenous liquidity differences

I report how the results change if the share of GDP produced by traded "trees" is in fact larger, say 0.45. (Recall that the gross value added of the nonfinancial corporate business sector as a share of GDP, i.e., the upper bound on the relevant target, is about 0.5.) Table 8 corresponds to the economy with $\kappa=2 / 9$ and $\hat{B}=0.6658$, which imply that tradeable trees produce $45 \%$ of GDP and that the average bond to GDP ratio is 0.3 (as in the benchmark) and $\theta=0$. For this parametrization, the liquidity constraints start binding for $\sigma=4$. The equilibrium for lower values of $\sigma$ (and hence all entries in the table) are just as in Mehra-Prescott. If we compare Table 9 with Table 3, it is apparent that liquidity constraints are tighter in the economy with 
lower $\kappa \cdot{ }^{41}$ First, they start binding for lower values of $\sigma$ (i.e., 4 as opposed to 8 ), and second, they are tighter when they both bind. (This is evident from comparing the magnitudes of the Euler equation wedges in Table 9 to their counterparts in Table 3.) Tighter liquidity constraints imply that equity and bond returns are uniformly lower in this economy than in the one with higher $\kappa$. For $\sigma=9$ or larger, agents are so eager for liquidity that they are willing to hold bonds even though their measured return is negative on average (bonds still sell at a discount in the high state).

Table 10 reports the results for the model with exogenous liquidity differences. For $\sigma=3$ and lower, the first four columns are the same as those of Table 4. But for each value of $\sigma$, the implied values of $\theta$ are uniformly lower in Table 10, the case with smaller $\kappa$. For $\sigma=6$ the liquidity needs are so severe that there is no positive $\theta$ that can induce a risk-free rate as high as $1 \%$. To summarize, the liquidity mechanism is magnified in parametrizations with smaller values of $\kappa$. This implies that somewhat smaller values of $\theta$ are needed to account for the equity premium. $^{42}$

Higher values of $\kappa$ tend to relax the liquidity constraints, and in fact, they will bind in at least some state of the world iff $\kappa<\bar{\kappa}$, where

$$
\bar{\kappa}=\left[\frac{1-(1+\epsilon)^{1-\sigma}}{(\sigma-1) \min _{i, j}\left(B_{i} / \gamma_{j}\right)}\right]^{\frac{1}{\sigma-1}} .
$$

Given that the rest of the parameters and data targets are as in the benchmark, I found that for targets of the share of GDP produced by trees with tradeable equity that are less than $35 \%$, there are no liquidity needs in any state. ${ }^{43}$

\footnotetext{
${ }^{41}$ With CRRA preferences, the difference $u[(1+\epsilon) \kappa]-u(\kappa)$ is decreasing in $\kappa$ for $\sigma>1$. This difference is essentially what determines the size of the right-hand side of the inequalities that appear in the definitions of the sets $\Omega$ and $\Omega_{\theta}$ in (20) and (21). Thus, the liquidity mechanism is stronger for smaller $\kappa$.

${ }^{42}$ The volume bounds do not appear too sensitive, e.g., those corresponding to $\sigma=3$ are now [.69, 20].

${ }^{43}$ If this target is set slightly higher - at 36\%, for example - then the liquidity constraints are slack in all trades, even those where only bonds can be used, up to $\sigma=7$. For $\sigma=8$ and $\sigma=9$, setting $\theta=1$ is not enough of a liquidity advantage on bonds to bring their return down to $1 \%$. With $\sigma=8$ and $\theta=1$, the risk-free rate is $11.27 \%$, virtually as high as in the Mehra-Prescott economy. With $\sigma=9$ and $\theta=1$, the risk-free rate is $6 \%$, half of what it would be for Mehra-Prescott, but still high. With $\sigma=10$, setting $\theta=0.7$ induces a risk-free rate of $1 \%$, and the return on equity is $15.79 \%$, essentially the same as in Mehra-Prescott. (With $\sigma=10, \theta=0.7$ implies $[\underline{v}, \bar{v}]=[9,62]$.
} 


\begin{tabular}{ccccc}
\hline \hline$\sigma$ & $\begin{array}{c}\text { Equity } \\
\text { Return }\end{array}$ & $\begin{array}{c}\text { Bond } \\
\text { Return }\end{array}$ & $\begin{array}{c}\text { Equity } \\
\text { Premium }\end{array}$ & $\begin{array}{c}\text { EE Wedges } \\
\omega^{e}, \omega^{b}\end{array}$ \\
\hline 1 & 2.83 & 2.70 & 0.13 & 0,0 \\
2 & 4.58 & 4.30 & 0.28 & 0,0 \\
3 & 6.27 & 5.79 & 0.48 & 0,0 \\
4 & 6.33 & 5.58 & 0.75 & $.0003,-.0149$ \\
5 & 3.15 & 2.13 & 1.02 & $.0006,-.0584$ \\
6 & 2.29 & 0.96 & 1.33 & $.0008,-.0790$ \\
7 & 2.10 & 0.43 & 1.67 & $.0011,-.0926$ \\
8 & 2.08 & 0.04 & 2.04 & $.0014,-.1036$ \\
9 & 2.12 & -0.31 & 2.43 & $.0016,-.1133$ \\
10 & 2.18 & -0.67 & 2.85 & $.0019,-.1219$ \\
\hline
\end{tabular}

Table 9: Robustness, lower $\kappa$ with $\theta=0$

\begin{tabular}{cccccc}
\hline \hline$\sigma$ & $\begin{array}{c}\text { Equity } \\
\text { Return }\end{array}$ & $\begin{array}{c}\text { Bond } \\
\text { Return }\end{array}$ & $\begin{array}{c}\text { Equity } \\
\text { Premium }\end{array}$ & $\begin{array}{c}\text { EE Wedges } \\
\omega^{e}, \omega^{b}\end{array}$ & $\theta^{*}$ \\
\hline 1 & 2.83 & 2.70 & 0.13 & 0,0 & - \\
2 & 4.58 & 1 & 3.58 & $.0316,-.0316$ & .0260 \\
3 & 6.27 & 1 & 5.27 & $.0453,-.0453$ & $.022 \times 10^{-1}$ \\
4 & 6.33 & 1 & 5.33 & $.0430,-.0576$ & $.011 \times 10^{-2}$ \\
5 & 3.15 & 1 & 2.15 & $.0110,-.0687$ & $17 \times 10^{-7}$ \\
6 & 2.29 & 0.96 & 1.33 & $.0008,-.0790$ & 0 \\
\hline
\end{tabular}

Table 10: Robustness, lower $\kappa$ with exogenous liquidity differences

In the baseline calibration, I use $\epsilon=0.8$. Now suppose $\epsilon=0.5$, and keep all other parameters as in the benchmark. Tables 11 and 12 are very similar to Tables 3 and 4, so the key results appear robust to lowering $\varepsilon$. The volume bounds implied by the values of $\theta$ corresponding to $\sigma=5,4$, and 3 in Table 12 are $[.05,3],[.3,8]$, and [3, 43], respectively.

Next, consider the parametrization with $\epsilon=1$, and keep all other parameters at their benchmark values. The results for the case with $\theta=0$ and for the one where $\theta$ is chosen so that the risk-free rate is $1 \%$ are reported in Tables 13 and 14, respectively. The results do not seem to be altered much by increasing $\epsilon$ away from its benchmark value. For example, in Table 14, the volume bounds implied by the values of $\theta$ corresponding to $\sigma=5,4$, and 3 are $[.007,2.4]$, $[.05,5.75]$, and $[.39,16]$, respectively. 


\begin{tabular}{ccccc}
\hline \hline$\sigma$ & $\begin{array}{c}\text { Equity } \\
\text { Return }\end{array}$ & $\begin{array}{c}\text { Bond } \\
\text { Return }\end{array}$ & $\begin{array}{c}\text { Equity } \\
\text { Premium }\end{array}$ & $\begin{array}{c}\text { EE Wedges } \\
\omega^{e}, \omega^{b}\end{array}$ \\
\hline 1 & 2.83 & 2.70 & 0.13 & 0,0 \\
2 & 4.58 & 4.30 & 0.28 & 0,0 \\
3 & 6.27 & 5.79 & 0.48 & 0,0 \\
4 & 7.89 & 7.18 & 0.71 & 0,0 \\
5 & 9.42 & 8.45 & 0.97 & 0,0 \\
6 & 10.88 & 9.62 & 1.26 & 0,0 \\
7 & 12.24 & 10.67 & 1.57 & 0,0 \\
8 & 9.13 & 7.09 & 2.04 & $.0009,-.0405$ \\
9 & 6.11 & 3.69 & 2.42 & $.0013,-.0777$ \\
10 & 4.41 & 1.57 & 2.84 & $.0017,-.1021$ \\
\hline
\end{tabular}

Table 11: Robustness, $\epsilon=0.5$ with $\theta=0$

\begin{tabular}{cccccc}
\hline \hline$\sigma$ & $\begin{array}{c}\text { Equity } \\
\text { Return }\end{array}$ & $\begin{array}{c}\text { Bond } \\
\text { Return }\end{array}$ & $\begin{array}{c}\text { Equity } \\
\text { Premium }\end{array}$ & $\begin{array}{c}\text { EE Wedges } \\
\omega^{e}, \omega^{b}\end{array}$ & $\theta^{*}$ \\
\hline 1 & 2.83 & 2.70 & 0.13 & 0,0 & - \\
2 & 4.58 & 4.30 & 0.28 & 0,0 & - \\
3 & 6.27 & 1 & 5.27 & $.0453,-.0453$ & .218 \\
4 & 7.89 & 1 & 6.89 & $.0576,-.0576$ & .060 \\
5 & 9.42 & 1 & 8.42 & $.0687,-.0687$ & .020 \\
6 & 10.88 & 1 & 9.88 & $.0786,-.0786$ & $0.06 \times 10^{-1}$ \\
7 & 12.24 & 1 & 11.24 & $.0873,-.0873$ & $0.02 \times 10^{-1}$ \\
8 & 9.13 & 1 & 8.13 & $.0555,-.0950$ & $3.49 \times 10^{-4}$ \\
9 & 6.11 & 1 & 5.11 & $.0252,-.1016$ & $4.20 \times 10^{-5}$ \\
10 & 4.41 & 1 & 3.41 & $.0067,-.1072$ & $2.38 \times 10^{-6}$ \\
\hline
\end{tabular}

Table 12: Robustness, $\epsilon=0.5$ with exogenous liquidity differences

The choice of $\epsilon$ has implications for consumption inequality. In the benchmark calibration with $\epsilon=0.8$, the variance of the log of total (i.e., including general goods and fruit in both subperiods) per capita consumption is 0.013 , and the variance of the log of per capita consumption in the first subperiod (which is the only source of consumption inequality in the model) is 0.67 . The parametrization with $\epsilon=0.5$ used to generate Tables 11 and 12 implies that the variance of the log of total consumption equals 0.005 , whereas the variance of the log of consumption in the first subperiod equals 0.156. (See footnote 22.) 


\begin{tabular}{ccccc}
\hline \hline$\sigma$ & $\begin{array}{c}\text { Equity } \\
\text { Return }\end{array}$ & $\begin{array}{c}\text { Bond } \\
\text { Return }\end{array}$ & $\begin{array}{c}\text { Equity } \\
\text { Premium }\end{array}$ & $\begin{array}{c}\text { EE Wedges } \\
\omega^{e}, \omega^{b}\end{array}$ \\
\hline 1 & 2.8301 & 2.7038 & .1262 & 0,0 \\
2 & 4.5842 & 4.2973 & .2868 & 0,0 \\
3 & 6.2722 & 5.7905 & .4817 & 0,0 \\
4 & 7.8871 & 7.1774 & .7097 & 0,0 \\
5 & 9.4238 & 8.4542 & .9696 & 0,0 \\
6 & 10.8780 & 9.6180 & 1.260 & 0,0 \\
7 & 12.2454 & 10.6663 & 1.579 & 0,0 \\
8 & 8.7428 & 6.6923 & 2.0506 & $.00100,-.04400$ \\
9 & 5.9525 & 3.5163 & 2.4362 & $.00138,-.07924$ \\
10 & 4.3440 & 1.4950 & 2.8489 & $.00170,-. .10280$ \\
\hline
\end{tabular}

Table 13: Robustness, $\epsilon=1$ with $\theta=0$

\begin{tabular}{cccccc}
\hline \hline$\sigma$ & $\begin{array}{c}\text { Equity } \\
\text { Return }\end{array}$ & $\begin{array}{c}\text { Bond } \\
\text { Return }\end{array}$ & $\begin{array}{c}\text { Equity } \\
\text { Premium }\end{array}$ & $\begin{array}{c}\text { EE Wedges } \\
\omega^{e}, \omega^{b}\end{array}$ & $\theta^{*}$ \\
\hline 1 & 2.83 & 2.70 & 0.13 & 0,0 & - \\
2 & 4.58 & 1 & 3.58 & $.0316,-.0316$ & .1693 \\
3 & 6.27 & 1 & 5.27 & $.0453,-.0453$ & .0467 \\
4 & 7.89 & 1 & 6.89 & $.0576,-.0576$ & .0140 \\
5 & 9.42 & 1 & 8.42 & $.0687,-.0687$ & $.037 \times 10^{-1}$ \\
6 & 10.88 & 1 & 9.88 & $.0786,-.0786$ & $.083 \times 10^{-2}$ \\
7 & 12.24 & 1 & 11.24 & $.0873,-.0873$ & $1.62 \times 10^{-4}$ \\
8 & 8.74 & 1 & 7.74 & $.0520,-.0950$ & $1.57 \times 10^{-5}$ \\
9 & 5.95 & 1 & 4.95 & $.0238,-.1016$ & $1.02 \times 10^{-6}$ \\
10 & 4.34 & 1 & 3.34 & $.0061,-.1072$ & $2.71 \times 10^{-8}$ \\
\hline
\end{tabular}

Table 14: Robustness, $\epsilon=1$ with exogenous liquidity differences

\section{A.5 Generalized Nash bargaining}

So far, in the determination of the terms of trade in decentralized meetings, I have focused on the formulation in which the buyer (the agent with the low endowment) makes a take-it-or-leave-it offer. Here I show how to generalize the theoretical analysis to the case where the terms of trade are instead determined according to the Nash bargaining solution with bargaining power $\eta \in[0,1]$ for the buyer and $1-\eta$ for the seller. At the end of this section, I also report how the baseline quantitative results of Section 4.2, which assumed $\eta=1$, depend on the value of $\eta$.

As it will become clear below, having $\eta<1$ introduces the possibility that buyers may 
be better off not bringing all the assets they own to the bargaining table. For this reason, I generalize the model to allow agents to choose which part of their portfolios to bring into a meeting. For $j=h, l$, let $\hat{V}_{j}(\mathbf{a}, \mathbf{s})=\max _{\hat{\mathbf{a}} \leq \mathbf{a}} V_{j}(\hat{\mathbf{a}}, \mathbf{s})$, where $V_{h}(\mathbf{a}, \mathbf{s})$ and $V_{l}(\mathbf{a}, \mathbf{s})$ are as in Section 2 , and $\hat{\mathbf{a}}=\left(\hat{a}^{b}, \hat{a}^{s}\right)$ is the portfolio that the agent chooses to bring into the random matching round. (The timing is that the agent chooses â right before the bargaining takes place, but after having learned the realization of the aggregate shock, his individual endowment realization, and the type of match he will be in.) Explicitly,

$$
\begin{aligned}
\hat{V}_{h}(\mathbf{a}, \mathbf{s})= & \frac{\hat{\alpha}}{2} \sum_{i=1,2} \theta_{i} \max _{\hat{\mathbf{a}} \leq \mathbf{a}}\left[\int\left\{u\left[(1+\epsilon) \kappa d-q^{i}(\tilde{\mathbf{a}}, \hat{\mathbf{a}})\right]+W\left[\mathbf{a}+\mathbf{p}^{i}(\tilde{\mathbf{a}}, \hat{\mathbf{a}}), \mathbf{s}\right]\right\} d \mathbf{G}(\tilde{\mathbf{a}})\right] \\
& +\left(1-\frac{\hat{\alpha}}{2}\right)\{u[(1+\epsilon) \kappa d]+W(\mathbf{a}, \mathbf{s})\} \\
\hat{V}_{l}(\mathbf{a}, \mathbf{s})= & \frac{\hat{\alpha}}{2} \sum_{i=1,2} \theta_{i} \max _{\hat{\mathbf{a}} \leq \mathbf{a}}\left[\int\left\{u\left[(1-\epsilon) \kappa d+q^{i}(\hat{\mathbf{a}}, \tilde{\mathbf{a}})\right]+W\left[\mathbf{a}-\mathbf{p}^{i}(\hat{\mathbf{a}}, \tilde{\mathbf{a}}), \mathbf{s}\right]\right\} d \mathbf{G}(\tilde{\mathbf{a}})\right] \\
& +\left(1-\frac{\hat{\alpha}}{2}\right)\{u[(1-\epsilon) \kappa d]+W(\mathbf{a}, \mathbf{s})\} .
\end{aligned}
$$

The value of an agent who enters the centralized market holding portfolio $\mathbf{a}_{t}$ in a period when the aggregate state of the economy is $\mathbf{s}_{t}$, namely, $W\left(\mathbf{a}_{t}, \mathbf{s}_{t}\right)$, is still given by (1), but with $V(\mathbf{a}, \mathbf{s})=\frac{1}{2}\left[\hat{V}_{l}(\mathbf{a}, \mathbf{s})+\hat{V}_{h}(\mathbf{a}, \mathbf{s})\right] ;$ i.e.,

$$
\begin{aligned}
V(\mathbf{a}, \mathbf{s})= & \alpha \sum_{i=1,2} \theta_{i} \max _{\hat{\mathbf{a}} \leq \mathbf{a}}\left[\int\left\{u\left[(1-\epsilon) \kappa d+q^{i}(\hat{\mathbf{a}}, \tilde{\mathbf{a}})\right]+W\left[\mathbf{a}-\mathbf{p}^{i}(\hat{\mathbf{a}}, \tilde{\mathbf{a}}), \mathbf{s}\right]\right\} d \mathbf{G}(\tilde{\mathbf{a}})\right] \\
& +\alpha \sum_{i=1,2} \theta_{i} \max _{\hat{\mathbf{a}} \leq \mathbf{a}}\left[\int\left\{u\left[(1+\epsilon) \kappa d-q^{i}(\tilde{\mathbf{a}}, \hat{\mathbf{a}})\right]+W\left[\mathbf{a}+\mathbf{p}^{i}(\tilde{\mathbf{a}}, \hat{\mathbf{a}}), \mathbf{s}\right]\right\} d \mathbf{G}(\tilde{\mathbf{a}})\right] \\
& +(1-2 \alpha)[\bar{u}(d)+W(\mathbf{a}, \mathbf{s})] .
\end{aligned}
$$

Consider a meeting of type $i$ in the decentralized market between a buyer who owns portfolio $\mathbf{a}$ and brings $\hat{\mathbf{a}} \leq \mathbf{a}$ to the meeting, and a seller who owns $\tilde{\mathbf{a}}$ and brings $\tilde{\mathbf{a}}^{\prime}$ to the meeting. The terms of trade $\left[q^{i}\left(\hat{\mathbf{a}}, \tilde{\mathbf{a}}^{\prime}\right), \mathbf{p}^{i}\left(\hat{\mathbf{a}}, \tilde{\mathbf{a}}^{\prime}\right)\right]$, for $i=1,2$, are determined by Nash bargaining. Let $u_{b}(q) \equiv u[(1-\epsilon) \kappa d+q]-u[(1-\epsilon) \kappa d]$ and $c_{s}(q) \equiv u[(1+\epsilon) \kappa d]-u[(1+\epsilon) \kappa d-q]$. Then, for $i=1,2$, the terms of trade $\left(q^{i}, \mathbf{p}^{i}\right)$ maximize

$$
\left[u_{b}\left(q^{i}\right)+W\left(\mathbf{a}-\mathbf{p}^{i}, \mathbf{s}\right)-W(\mathbf{a}, \mathbf{s})\right]^{\eta}\left[-c_{s}\left(q^{i}\right)+W\left(\tilde{\mathbf{a}}+\mathbf{p}^{i}, \mathbf{s}\right)-W(\tilde{\mathbf{a}}, \mathbf{s})\right]^{1-\eta}
$$


subject to $\mathbf{p}^{1}=\left(p^{b}, 0\right)$ and $p^{b} \leq \hat{a}^{b}$ if $i=1$ (matches where only bonds can be used as means of payment), or subject to $\mathbf{p}^{2}=\left(p^{b}, p^{s}\right) \leq \hat{\mathbf{a}}$ if $i=2$ (matches where both bonds and shares can be used for payment). In an unrestricted match, the agent cannot spend more than all the assets he chooses to bring into the meeting. And in a restricted match, he cannot spend more than all the bonds he brings into the meeting. Let

$$
q(z ; \kappa d)= \begin{cases}\epsilon \kappa d & \text { if } z \geq g_{\eta}(\epsilon \kappa d) \\ q_{\eta}(z ; \kappa d) & \text { if } z<g_{\eta}(\epsilon \kappa d)\end{cases}
$$

where

$$
g_{\eta}(q)=\frac{\eta u_{b}^{\prime}(q) c_{s}(q)+(1-\eta) c_{s}^{\prime}(q) u_{b}(q)}{\eta u_{b}^{\prime}(q)+(1-\eta) c_{s}^{\prime}(q)}
$$

and $q_{\eta}(z ; \kappa d)$ denotes the $q$ that solves $g_{\eta}(q)=z$. For $i=1,2$, the bargaining solution in period $t$ is $q^{i}\left(\hat{\mathbf{a}}_{t}, \tilde{\mathbf{a}}_{t}^{\prime}\right)=q\left(\boldsymbol{\lambda}_{t}^{i} \hat{\mathbf{a}}_{t} ; \kappa d_{t}\right)$, where $\boldsymbol{\lambda}_{t}^{1} \equiv\left(\lambda_{t}^{b}, 0\right)$, and $\boldsymbol{\lambda}_{t}^{2}=\boldsymbol{\lambda}_{t}$. Intuitively, $q(z ; \kappa d)$ is the quantity of coconuts that get traded in a meeting in which the buyer brings portfolio with real value $z$ to the bargaining table, during a period when the aggregate endowment of coconuts is $\kappa d$. Differentiation implies

$$
\frac{\partial q(z ; \kappa d)}{\partial z}=\frac{1}{g_{\eta}^{\prime}(q)}
$$

where

$$
g_{\eta}^{\prime}(q)=\frac{\eta c_{s}^{\prime}(q)\left[u_{b}^{\prime}(q)\right]^{2}+(1-\eta) u_{b}^{\prime}(q)\left[c_{s}^{\prime}(q)\right]^{2}+\eta(1-\eta)\left[c_{s}^{\prime \prime}(q) u_{b}^{\prime}(q)-u_{b}^{\prime \prime}(q) c_{s}^{\prime}(q)\right]\left[u_{b}(q)-c_{s}(q)\right]}{\left[\eta u_{b}^{\prime}(q)+(1-\eta) c_{s}^{\prime}(q)\right]^{2}} .
$$

Using the fact that $W\left[\mathbf{a}+\mathbf{p}^{i}(\mathbf{a}, \tilde{\mathbf{a}}), \mathbf{s}\right]=W(\mathbf{a}, \mathbf{s})+\lambda \mathbf{p}^{i}(\mathbf{a}, \tilde{\mathbf{a}})$ and the bargaining solution, the expected value of search can be written more compactly:

$$
V(\mathbf{a}, \mathbf{s})=W(\mathbf{a}, \mathbf{s})+\alpha \sum_{i=1,2} \theta_{i} \max _{\hat{\mathbf{a}} \leq \mathbf{a}} \Gamma_{b}\left(\boldsymbol{\lambda}^{i} \hat{\mathbf{a}}\right)+\alpha \Gamma_{s}+\bar{u}(d)
$$

where

$$
\Gamma_{b}(z) \equiv u_{b}[q(z ; \kappa d)]-g_{\eta}[q(z ; \kappa d)]
$$

is expected gain from trade when the agent acts as a buyer and carries a portfolio with real value $z$ into the bilateral match, and $\Gamma_{s} \equiv \sum_{i=1,2} \theta_{i} \int\left\{-c_{s}\left[q\left(\boldsymbol{\lambda}^{i} \tilde{\mathbf{a}} ; \kappa d\right)\right]+g_{\eta}\left[q\left(\boldsymbol{\lambda}^{i} \tilde{\mathbf{a}} ; \kappa d_{t}\right)\right]\right\} d \mathbf{G}(\tilde{\mathbf{a}})$ is the expected gain from trade when he acts as a seller. 
Consider the problem $\max _{z} \Gamma_{b}(z)$, i.e., the unconstrained version of the maximization problems in the second and third terms of (31). First, notice that according to the bargaining solution, $\Gamma_{b}(z)$ is constant for all $z \geq g_{\eta}(\epsilon \kappa d)$. Moreover, note that as $z$ approaches $g_{\eta}(\epsilon \kappa d)$ from below,

$$
g_{\eta}^{\prime}(q) \rightarrow u^{\prime}(\kappa d)+\eta(1-\eta) \frac{-4 u^{\prime \prime}(\kappa d)}{u^{\prime}(\kappa d)}\left\{u(\kappa d)-\frac{u[(1-\epsilon) \kappa d]+u[(1+\epsilon) \kappa d]}{2}\right\} .
$$

Thus, $\lim _{z \uparrow g_{\eta}(\epsilon \kappa d)} \Gamma_{b}^{\prime}(z)=\eta(1-\eta) \frac{4 u^{\prime \prime}(\kappa d)}{u^{\prime}(\kappa d)}\left\{u(\kappa d)-\frac{u[(1-\epsilon) \kappa d]+u[(1+\epsilon) \kappa d]}{2}\right\} \leq 0$, with strict inequality unless $\eta=1$. Therefore, $\max _{z \geq 0} \Gamma_{b}(z)=\max _{0 \leq z \leq g_{\eta}(\epsilon \kappa d)} \Gamma_{b}(z)$. Since the objective function is continuous and the choice set compact, a solution $z_{b}^{*}$ exists for this problem. Given $z_{b}^{*}$, let $q_{b}^{*}$ be defined by $g_{\eta}\left(q_{b}^{*}\right)=z_{b}^{*}$. Intuitively, $q_{b}^{*}$ is the quantity traded that maximizes the buyer's gains from trade in the bilateral meeting, and $z_{b}^{*}$ is the real value of assets needed to purchase this quantity.

The previous reasoning implies that $z_{b}^{*}<g_{\eta}(\epsilon \kappa d)$ and $q_{b}^{*}<\epsilon \kappa d$ for $\eta \in[0,1)$, but $q_{b}^{*}=\epsilon \kappa d$ if $\eta=1$. Thus, the solution to $\max _{\hat{\mathbf{a}} \leq \mathbf{a}} \Gamma_{b}\left(\boldsymbol{\lambda}^{i} \hat{\mathbf{a}}\right)$ takes the following form. If $\boldsymbol{\lambda}^{i} \mathbf{a} \geq g_{\eta}\left(q_{b}^{*}\right)$, then the buyer only brings a portfolio $\hat{\mathbf{a}} \leq \mathbf{a}$ such that $\boldsymbol{\lambda}^{i} \hat{\mathbf{a}}=g_{\eta}\left(q_{b}^{*}\right)$ to the bilateral match, and purchases $q_{b}^{*}$ from the seller. (If $\eta=1$, he brings any portfolio $\hat{\mathbf{a}} \leq \mathbf{a}$ such that $\boldsymbol{\lambda}^{i} \hat{\mathbf{a}} \geq u[(1+\epsilon) \kappa d]-u(\kappa d)$ and purchases $\epsilon \kappa d$ from the seller.) Conversely, if $\boldsymbol{\lambda}^{i} \mathbf{a}<g_{\eta}\left(q_{b}^{*}\right)$, then the buyer brings all the assets he owns into the bilateral meeting, and he purchases $q_{\eta}\left(\boldsymbol{\lambda}^{i} \mathbf{a} ; \kappa d\right)<q_{b}^{*} .{ }^{44}$ Let

$$
q_{b}(z ; \kappa d)= \begin{cases}q_{b}^{*} & \text { if } z \geq g_{\eta}\left(q_{b}^{*}\right) \\ q_{\eta}(z ; \kappa d) & \text { if } z<g_{\eta}\left(q_{b}^{*}\right)\end{cases}
$$

Intuitively, $q_{b}(z ; \kappa d)$ is the quantity of coconuts that get traded in a meeting between a seller and a buyer who owns portfolio with real value $z$. This is to be contrasted with $q(z ; \kappa d)$, which was the quantity of coconuts that get traded in a meeting where the buyer brings portfolio with real value $z$ to the bargaining table. Thus, for $i=1,2$, the bargaining solution in period

\footnotetext{
${ }^{44}$ This second part presumes that $\Gamma_{b}(z)$ is nondecreasing on $\left[0, z_{b}^{*}\right]$, as will be the case in the parametrizations I will consider. Otherwise the agent may sometimes prefer not to bring all the assets he owns into the bilateral bargain, even though $\boldsymbol{\lambda}^{i} \mathbf{a}<g_{\eta}\left(q_{b}^{*}\right)$. More generally, the statement is that if $\boldsymbol{\lambda}^{i} \mathbf{a}<g_{\eta}\left(q_{b}^{*}\right)$, then the buyer brings into the bilateral match a portfolio $\hat{\mathbf{a}} \leq \mathbf{a}$ such that $\boldsymbol{\lambda}^{i} \hat{\mathbf{a}}=\tilde{z}_{b}$, where $\tilde{z}_{b}=\arg \max _{0 \leq z \leq \boldsymbol{\lambda}^{i} \mathbf{a}} \Gamma_{b}(z)$, and he purchases $q_{\eta}\left(\tilde{z}_{b} ; \kappa d\right)<q_{b}^{*}$.
} 
$t$, taking into account the buyer's choice of which portfolio to bring to the bargaining table, implies that - in a period when the aggregate endowment of coconuts is $\kappa d_{t}$ - the quantity traded between a buyer who owns portfolio $\mathbf{a}_{t}$ and any seller is $q_{b}\left(\boldsymbol{\lambda}_{t}^{i} \mathbf{a}_{t} ; \kappa d_{t}\right)$, where $\boldsymbol{\lambda}_{t}^{1} \equiv\left(\lambda_{t}^{b}, 0\right)$ and $\lambda_{t}^{2}=\lambda_{t}$.

I can now write (31) as

$$
V(\mathbf{a}, \mathbf{s})=\alpha \sum_{i=1,2} \theta_{i} \mathcal{S}_{b}\left(\boldsymbol{\lambda}^{i} \mathbf{a} ; d\right)+W(\mathbf{a}, \mathbf{s})+\alpha \Gamma_{s}+\bar{u}(d)
$$

where $\mathcal{S}_{b}\left(\boldsymbol{\lambda}^{i} \mathbf{a} ; d\right)=u_{b}\left[q_{b}\left(\boldsymbol{\lambda}^{i} \mathbf{a} ; \kappa d\right)\right]-g_{\eta}\left[q_{b}\left(\boldsymbol{\lambda}^{i} \mathbf{a} ; \kappa d\right)\right]$. Then, focus on the agent's maximization problem in the centralized market, i.e., (1). The optimal choices of $c_{t}$ and $n_{t}$ still satisfy (24) and (25). The portfolio choice, $\mathbf{a}_{t+1}$, satisfies (26), but now,

$$
\begin{aligned}
& \frac{\partial V\left(\mathbf{a}_{t+1}, \mathbf{s}_{t+1}\right)}{\partial a_{t+1}^{b}}=\left[1+\alpha \sum_{i=1,2} \theta_{i}\left(\frac{u_{b}^{\prime}\left[q_{b}\left(\boldsymbol{\lambda}_{t+1}^{i} \mathbf{a}_{t+1} ; \kappa d_{t+1}\right)\right]}{g_{\eta}^{\prime}\left[q_{b}\left(\boldsymbol{\lambda}_{t+1}^{i} \mathbf{a}_{t+1} ; \kappa d_{t+1}\right)\right]}-1\right)\right] \lambda_{t+1}^{b}, \\
& \frac{\partial V\left(\mathbf{a}_{t+1}, \mathbf{s}_{t+1}\right)}{\partial a_{t+1}^{s}}=\left[1+\alpha \theta_{2}\left(\frac{u_{b}^{\prime}\left[q_{b}\left(\boldsymbol{\lambda}_{t+1} \mathbf{a}_{t+1} ; \kappa d_{t+1}\right)\right]}{g_{\eta}^{\prime}\left[q_{b}\left(\boldsymbol{\lambda}_{t+1} \mathbf{a}_{t+1} ; \kappa d_{t+1}\right)\right]}-1\right)\right] \lambda_{t+1}^{s},
\end{aligned}
$$

are obtained by differentiating (32). These conditions, together with $\lambda_{t}^{1}=\left(\lambda_{t}^{b}, 0\right), \lambda_{t}^{2}=\left(\lambda_{t}^{b}, \lambda_{t}^{s}\right)$, $\lambda_{t}^{b}=\frac{A_{t}}{w_{t}}$, and $\lambda_{t}^{s}=\left(\phi_{t}^{s}+d_{t}\right) \lambda_{t}^{b}$, imply that the Euler equation $U^{\prime}\left(c_{t}\right) \phi_{t}^{i}=\beta E_{t} \frac{\partial V\left(\mathbf{a}_{t+1}, \mathbf{s}_{t+1}\right)}{\partial a_{t+1}^{i}}$ can be written as

$$
\begin{aligned}
U^{\prime}\left(d_{t}\right) \phi_{t}^{s} & =\beta E_{t} U^{\prime}\left(d_{t+1}\right) L_{t+1}^{s}\left[\phi_{t+1}^{s}+d_{t+1}\right] \\
U^{\prime}\left(d_{t}\right) \phi_{t}^{b} & =\beta E_{t} U^{\prime}\left(d_{t+1}\right) L_{t+1}^{b}
\end{aligned}
$$

with

$$
\begin{aligned}
& L_{t+1}^{s}=1+\alpha(1-\theta)\left\{\frac{\left.u_{b}^{\prime}\left(q_{b}\left[U^{\prime}\left(d_{t+1}\right)\left(\phi_{t+1}^{s}+d_{t+1}+B_{t+1}\right) ; \kappa d_{t+1}\right)\right]\right)}{\left.g_{\eta}^{\prime}\left(q_{b}\left[U^{\prime}\left(d_{t+1}\right)\left(\phi_{t+1}^{s}+d_{t+1}+B_{t+1}\right) ; \kappa d_{t+1}\right)\right]\right)}-1\right\} \\
& L_{t+1}^{b}=L_{t+1}^{s}+\alpha \theta\left\{\frac{u_{b}^{\prime}\left(q_{b}\left[U^{\prime}\left(d_{t+1}\right) B_{t+1} ; \kappa d_{t+1}\right]\right)}{g_{\eta}^{\prime}\left(q_{b}\left[\left(U^{\prime}\left(d_{t+1}\right) B_{t+1} ; \kappa d_{t+1}\right]\right)\right.}-1\right\}
\end{aligned}
$$

where I have let $\theta \equiv \theta_{1}$ to simplify the notation. Note that (33)-(36) generalize (2)-(5) to the case of an arbitrary bargaining power $\eta \in[0,1]$. 
Now suppose that $U(c)=u(c)=\frac{c^{1-\sigma}}{1-\sigma}$, with $0<\sigma<\infty$. For this parametrization, $q_{b}\left(z ; \kappa d_{t+1}\right)$, i.e., the quantity of coconuts traded in a meeting where the buyer can pay from a portfolio of end-of-subperiod real value $z$ when the aggregate dividend is $d_{t+1}$, is given by

$$
q_{b}(z ; \kappa d)= \begin{cases}q_{b}^{*} & \text { if } z \geq g_{\eta}\left(q_{b}^{*}\right) \\ q_{\eta}(z ; \kappa d) & \text { if } z<g_{\eta}\left(q_{b}^{*}\right)\end{cases}
$$

where $q_{b}^{*}=\arg \max _{q}\left[u_{b}(q)-g_{\eta}(q)\right]$, and $q_{\eta}(z ; \kappa d)$ is the $q$ that solves

$$
g_{\eta}(q)=z
$$

but with $g_{\eta}(q)$ specialized to

$$
\begin{aligned}
g_{\eta}(q) \equiv & \frac{\eta[(1-\epsilon) \kappa d+q]^{-\sigma}}{\eta[(1-\epsilon) \kappa d+q]^{-\sigma}+(1-\eta)[(1+\epsilon) \kappa d-q]^{-\sigma}}\left\{\frac{[(1+\epsilon) \kappa d]^{1-\sigma}}{1-\sigma}-\frac{[(1+\epsilon) \kappa d-q]^{1-\sigma}}{1-\sigma}\right\}+ \\
& \frac{(1-\eta)[(1+\epsilon) \kappa d-q]^{-\sigma}}{\eta[(1-\epsilon) \kappa d+q]^{-\sigma}+(1-\eta)[(1+\epsilon) \kappa d-q]^{-\sigma}}\left\{\frac{[(1-\epsilon) \kappa d+q]^{1-\sigma}}{1-\sigma}-\frac{[(1-\epsilon) \kappa d]^{1-\sigma}}{1-\sigma}\right\} .
\end{aligned}
$$

I again specify that the government chooses the stock of bonds according to a policy rule $B_{t+1}=f\left(d_{t}, x_{t}\right)$, where $f\left(d_{t}, x_{t}\right)=\hat{B} E\left[d_{t+1} \mid\left(d_{t}, i\right)\right]$ if $x_{t}=\gamma_{i}$, and with $\hat{B} \geq 0$. Thus, if $d_{t+1}=x_{t+1} d_{t}$, with $x_{t}=\gamma_{i}$ and $x_{t+1}=\gamma_{j}$, we have $B_{t+1}=B_{i} d_{t}=\frac{B_{i}}{\gamma_{j}} d_{t+1}$. As before, I focus on equilibria that are stationary in growth rates, with share prices homogeneous of degree one in $d$, so that $\phi^{s}(d, j)=\phi_{j}^{s} d$, where $\phi_{j}^{s}$ is a constant. Also, this implies that $\phi^{b}(d, i)$ is independent of $d$, so I can write $\phi_{i}^{b}=\phi^{b}(d, i)$. Now consider a period $t+1$ in which $d_{t+1}=x_{t+1} d_{t}$, with $x_{t}=\gamma_{i}$ and $x_{t+1}=\gamma_{j}$. In such a period, the quantity traded in bilateral meetings is $\min \left\{q_{\eta}\left(z_{t+1} ; \kappa d_{t+1}\right), q_{b}^{*}\right\}$, with $z_{t+1}=\left(1+\phi_{j}^{s}+\frac{B_{i}}{\gamma_{j}}\right) d_{t+1}^{1-\sigma}$ in unrestricted matches and $z_{t+1}=\frac{B_{i}}{\gamma_{j}} d_{t+1}^{1-\sigma}$ in restricted matches.

The first observation is that just as in the case with $\eta=1$, the quantity traded when the buyer is unconstrained, i.e., $q_{b}^{*}$, is linear in the aggregate dividend, $d$. To see this, notice that choosing $q$ to maximize $u_{b}(q)-g_{\eta}(q)$ is equivalent to choosing $Q$ to maximize

$\frac{[(1-\epsilon) \kappa+Q]^{1-\sigma}}{1-\sigma}-\frac{\eta[(1-\epsilon) \kappa+Q]^{-\sigma}\left\{\frac{[(1+\epsilon) \kappa]^{1-\sigma}}{1-\sigma}-\frac{[(1+\epsilon) \kappa-Q]^{1-\sigma}}{1-\sigma}\right\}+(1-\eta)[(1+\epsilon) \kappa-Q]^{-\sigma}\left\{\frac{[(1-\epsilon) \kappa+Q]^{1-\sigma}}{1-\sigma}-\frac{[(1-\epsilon) \kappa]}{1-\sigma}\right\}}{\eta[(1-\epsilon) \kappa+Q]^{-\sigma}+(1-\eta)[(1+\epsilon) \kappa-Q]^{-\sigma}}$,

where $Q=q / d$. Let $\bar{q}_{b}^{*}$ denote the solution to the latter problem. Then, $q_{b}^{*}=\bar{q}_{b}^{*} d$. 
The second observation is that also as in the case with $\eta=1$, the quantity traded that solves (37) is linear in the aggregate dividend, $d$, both in restricted and in unrestricted matches. Consider restricted matches; i.e., set $z=\frac{B_{i}}{\gamma_{j}} d^{1-\sigma}$ in (37), and divide both sides by $d^{1-\sigma}$ to get

$$
\left.\frac{\eta[(1-\epsilon) \kappa+Q]^{-\sigma}\left\{\frac{[(1+\epsilon) \kappa]^{1-\sigma}}{1-\sigma}-\frac{[(1+\epsilon) \kappa-Q]}{1-\sigma}\right\}+(1-\eta)[(1+\epsilon) \kappa-Q]^{-\sigma}\left\{\frac{[(1-\epsilon) \kappa+Q]^{1-\sigma}}{1-\sigma}-\frac{[(1-\epsilon) \kappa]}{1-\sigma}\right\}}{\eta[(1-\epsilon) \kappa+Q]^{-\sigma}+(1-\eta)[(1+\epsilon) \kappa-Q]^{-\sigma}}\right\}=\frac{B_{i}}{\gamma_{j}},
$$

where $Q=q / d$. This equation implies that $Q$ is independent of $d$, or equivalently, that the quantity traded in restricted matches is $\min \left\{\bar{q}_{i j}^{1}, \bar{q}_{b}^{*}\right\} d$, where $\bar{q}_{i j}^{1}$ is the $Q$ that solves (38), which is independent of $d$. A similar argument implies that in unrestricted matches, the quantity traded is $\min \left\{\bar{q}_{i j}^{2}\left(\phi_{j}^{s}\right), \bar{q}_{b}^{*}\right\} d$, where $\bar{q}_{i j}^{2}\left(\phi_{j}^{s}\right)$ is the $Q$ that solves

$$
\frac{\eta[(1-\epsilon) \kappa+Q]^{-\sigma}\left\{\frac{[(1+\epsilon) \kappa]^{1-\sigma}}{1-\sigma}-\frac{[(1+\epsilon) \kappa-Q]^{1-\sigma}}{1-\sigma}\right\}+(1-\eta)[(1+\epsilon) \kappa-Q]^{-\sigma}\left\{\frac{[(1-\epsilon) \kappa+Q]^{1-\sigma}}{1-\sigma}-\frac{[(1-\epsilon) \kappa]^{1-\sigma}}{1-\sigma}\right\}}{\eta[(1-\epsilon) \kappa+Q]^{-\sigma}+(1-\eta)[(1+\epsilon) \kappa-Q]^{-\sigma}}=1+\phi_{j}^{s}+\frac{B_{i}}{\gamma_{j}},
$$

which is also independent of $d$. In other words, $q_{\eta}\left[\left(1+\phi_{j}^{s}+\frac{B_{i}}{\gamma_{j}}\right) d^{1-\sigma} ; \kappa d\right]=\bar{q}_{i j}^{2}\left(\phi_{j}^{s}\right) d$ in unrestricted matches, and $q_{\eta}\left(\frac{B_{i}}{\gamma_{j}} d^{1-\sigma} ; \kappa d\right)=\bar{q}_{i j}^{1} d$ in restricted matches.

Since $q_{b}\left[\left(1+\phi_{j}^{s}+\frac{B_{i}}{\gamma_{j}}\right) d^{1-\sigma} ; \kappa d\right]=\min \left\{\bar{q}_{i j}^{2}\left(\phi_{j}^{s}\right), \bar{q}_{b}^{*}\right\} d$ and $q_{b}\left(\frac{B_{i}}{\gamma_{j}} d^{1-\sigma} ; \kappa d\right)=\min \left\{\bar{q}_{i j}^{1}, \bar{q}_{b}^{*}\right\} d$, and (36) become

$$
\begin{aligned}
& L_{t+1}^{s}=1+\alpha(1-\theta)\left\{\frac{u_{b}^{\prime}\left(\min \left\{\bar{q}_{i j}^{2}\left(\phi_{j}^{s}\right), \bar{q}_{b}^{*}\right\} d_{t+1}\right)}{g_{\eta}^{\prime}\left(\min \left\{\bar{q}_{i j}^{2}\left(\phi_{j}^{s}\right), \bar{q}_{b}^{*}\right\} d_{t+1}\right)}-1\right\} \\
& L_{t+1}^{b}=L_{t+1}^{s}+\alpha \theta\left\{\frac{u_{b}^{\prime}\left(\min \left\{\bar{q}_{i j}^{1}, \bar{q}_{b}^{*}\right\} d_{t+1}\right)}{g_{\eta}^{\prime}\left(\min \left\{\bar{q}_{i j}^{1}, \bar{q}_{b}^{*}\right\} d_{t+1}\right)}-1\right\},
\end{aligned}
$$

where $u_{b}^{\prime}(q d)=[(1-\epsilon) \kappa+q]^{-\sigma} d^{-\sigma}$ and $g_{\eta}^{\prime}(q d)$ specializes to

$$
\left.\left\{\eta \Delta_{1}(q)[(1+\epsilon) \kappa-q]^{-\sigma}+(1-\eta) \Delta_{2}(q)[(1-\epsilon) \kappa+q)\right]^{-\sigma}+\eta(1-\eta) \Psi(q)\right\} d^{-\sigma}
$$

for $k=1,2$, with

$$
\Delta_{1}(q)=\left\{\frac{[(1-\epsilon) \kappa+q]^{-\sigma}}{\eta[(1-\epsilon) \kappa+q]^{-\sigma}+(1-\eta)[(1+\epsilon) \kappa-q]^{-\sigma}}\right\}^{2}, \Delta_{2}(q)=\left\{\frac{[(1+\epsilon) \kappa-q]^{-\sigma}}{\eta[(1-\epsilon) \kappa+q]^{-\sigma}+(1-\eta)[(1+\epsilon) \kappa-q]^{-\sigma}}\right\}^{2}
$$

and

$$
\begin{aligned}
\Psi(q)= & \frac{\left(\frac{\sigma}{1-\sigma}\right)\left\{[(1+\epsilon) \kappa-q]^{-\sigma-1}[(1-\epsilon) \kappa+q]^{-\sigma}+[(1-\epsilon) \kappa+q]^{-\sigma-1}[(1+\epsilon) \kappa-q]^{-\sigma}\right\}}{\left\{\eta[(1-\epsilon) \kappa+q]^{-\sigma}+(1-\eta)[(1+\epsilon) \kappa-q]^{-\sigma}\right\}} \times \\
& \frac{\left\{[(1-\epsilon) \kappa+q]^{1-\sigma}+[(1+\epsilon) \kappa-q]^{1-\sigma}-[(1-\epsilon) \kappa]^{1-\sigma}-[(1+\epsilon) \kappa]^{1-\sigma}\right\}}{\left\{\eta[(1-\epsilon) \kappa+q]^{-\sigma}+(1-\eta)[(1+\epsilon) \kappa-q]^{-\sigma}\right\}}
\end{aligned}
$$


Notice that $u_{b}^{\prime}\left(q d_{t+1}\right)=u_{b}^{\prime}(q) d_{t+1}^{-\sigma}$ and $g_{\eta}^{\prime}\left(q d_{t+1}\right)=g_{\eta}^{\prime}(q) d_{t+1}^{-\sigma}$, so the liquidity factors $L_{t+1}^{s}$ and $L_{t+1}^{b}$ in (39) and (40) are independent of $d_{t+1}$.

From (29) and (30), and (33)-(36), it is clear that as $\eta \rightarrow 1$, the equilibrium reduces to the one studied in Section 4. Conversely, as $\eta \rightarrow 0, g_{\eta}^{\prime}(q) \rightarrow u_{b}^{\prime}(q)$, and therefore $L_{i j}^{s} \rightarrow 1$ and $L_{i j}^{b} \rightarrow 1$ for all $i$ and $j$. If buyers have no bargaining power, assets have no value in exchange, and consequently, their value stems only from the streams of dividends they yield.

Table 15 reports the average equity return (ER), bond return (BR), and equity premium (EP) for $\eta=0,0.25,0.5,0.75$, and 1 , and values of $\sigma$ ranging from 1 to 10 . Table 15 generalizes Table 3, which assumed $\eta=1$.

\begin{tabular}{|c|c|c|c|c|c|c|c|c|c|c|c|c|c|c|c|}
\hline$\eta=$ & & 0 & & & 0.25 & & & 0.50 & & & 0.75 & & & 1 & \\
\hline$\sigma$ & ER & B R & EP & ER & BR & EP & ER & BR & EP & ER & B R & EP & ER & B R & EP \\
\hline 1 & 2.83 & 2.70 & 0.13 & 2.83 & 2.70 & 0.13 & 2.83 & 2.70 & 0.13 & 2.83 & 2.70 & 0.13 & 2.83 & 2.70 & 0.13 \\
\hline 2 & 4.58 & 4.30 & 0.28 & 4.58 & 4.30 & 0.28 & 4.58 & 4.30 & 0.28 & 4.58 & 4.30 & 0.28 & 4.58 & 4.30 & 0.28 \\
\hline 3 & 6.27 & 5.79 & 0.48 & 6.27 & 5.79 & 0.48 & 6.27 & 5.79 & 0.48 & 6.27 & 5.79 & 0.48 & 6.27 & 5.79 & 0.48 \\
\hline 4 & 7.89 & 7.18 & 0.71 & 7.89 & 7.18 & 0.71 & 7.89 & 7.18 & 0.71 & 7.89 & 7.18 & 0.71 & 7.89 & 7.18 & 0.71 \\
\hline 5 & 9.42 & 8.45 & 0.97 & 5.27 & 4.27 & 1.00 & 6.95 & 5.94 & 1.01 & 9.25 & 8.27 & 0.98 & 9.42 & 8.45 & 0.97 \\
\hline 6 & 10.88 & 9.62 & 1.26 & 3.09 & 1.80 & 1.29 & 3.69 & 2.40 & 1.29 & 4.59 & 3.30 & 1.29 & 10.88 & 9.62 & 1.26 \\
\hline 7 & 12.24 & 10.67 & 1.57 & 2.37 & 0.75 & 1.62 & 2.57 & 0.95 & 1.62 & 2.88 & 1.26 & 1.62 & 12.24 & 10.67 & 1.57 \\
\hline 8 & 13.52 & 11.60 & 1.92 & 2.17 & 0.20 & 1.97 & 2.23 & 0.26 & 1.97 & 2.33 & 0.36 & 1.97 & 8.80 & 6.76 & 2.04 \\
\hline 9 & 14.70 & 12.41 & 2.29 & 2.14 & -0.21 & 2.35 & 2.16 & -0.19 & 2.35 & 2.19 & -0.16 & 2.35 & 5.97 & 3.54 & 2.43 \\
\hline 10 & 15.79 & 13.10 & 2.69 & 2.17 & -0.58 & 2.75 & 2.18 & -0.57 & 2.75 & 2.19 & -0.56 & 2.75 & 4.35 & 1.50 & 2.85 \\
\hline
\end{tabular}

Table 15: Bargaining power in the baseline economy with $\theta=0$

First, note that as discussed above, $\eta=0$ implies that assets provide no liquidity services, i.e., that $L_{i j}^{s}=L_{i j}^{b}=1$ for all $i$ and $j$. Thus, for every value of $\sigma$, the asset returns corresponding to $\eta=0$ are identical to those of the Mehra-Prescott model reported in Table 2. Second, note that the asset returns in the column labeled $\eta=1$ correspond to the returns in Table 3 . For relatively low values of $\sigma$, e.g., $\sigma \leq 3$, the equilibrium is independent of $\eta$. In particular, the trading constraints are slack in every state of the world for these values of $\sigma$ (i.e., $L_{i j}^{s}=L_{i j}^{b}=1$ for all $i$ and $j$ ), so asset returns for $\sigma=1$ through 3 in Table 15 are the same as in the 
Mehra-Prescott economy of Table $2 .^{45}$

Recall that in Section 4.2 I reported that for $\sigma \leq 7$, the returns in the economy with $\eta=1$ were the same as in Mehra-Prescott. Table 15 shows that this is not the case for $\eta<1$. For example, consider the economy with $\sigma=5$. Note that asset returns are indeed the same for the Mehra-Prescott economy (e.g., $\eta=0)$ and the economy of Section $4.2(\eta=1)$. However, the equity and bond returns are U-shaped, and the equity premium is hump-shaped with respect to $\eta$. For example, the implied equity premium is $0.97 \%$ if $\eta=0,1 \%$ if $\eta=0.25,1.01 \%$ if $\eta=0.5,0.98 \%$ if $\eta=0.75$, and again $0.97 \%$ if $\eta=1$. The reason for this nonmonotonicity is that a reduction in $\eta$ has two opposing effects.

First, a smaller $\eta$ means that at the margin, the buyer gets fewer coconuts per unit of assets and reaps smaller gains from trade in decentralized exchanges, which tends to reduce the value of assets as a medium of exchange, and hence their liquidity return. But on the other hand, there is a corresponding general equilibrium effect, namely, that since assets are less valuable in exchange, their prices tend to be lower (agents would like to hold less of them, but they all have to be held in equilibrium), and this tends to tighten the trading constraints, so when they bind, assets are more valuable in exchange at the margin. The former effect tends to dominate for low values of $\eta$, and the latter for relatively large values. Interestingly, for intermediate values of $\sigma$, e.g., between (and including) 4 and 7, the equity premia reported in Section 4.2 for the case of $\eta=1$ are only lower bounds relative to the premia that can be generated with intermediate bargaining powers. Conversely, for relatively large values of $\sigma$, e.g., 8 through 10, for intermediate values of $\eta$ one finds equity premia that are smaller — if only slightly — than those of Section 4.2. For example, $2.75 \%$ instead of $2.85 \%$ for $\sigma=10$. From the results in Table 15, it seems reasonable to conclude that the findings reported in Section 4.2 are robust relative to the precise parametrization of the buyer's bargaining power.

\footnotetext{
${ }^{45}$ The equilibrium is not independent of $\eta$ for $\sigma=4$, despite the fact that the returns are the same across the values of $\eta$ reported in Table 15. For example, if $\sigma=4$ and $\eta=0.05$, then the equity return is $6.78 \%$, the bond return $6.04 \%$, and the equity premium $0.74 \%$. In this case, $\Omega\left(B_{i}\right)=\{1,2\}$ for all $i$. There are also more intricate binding patterns for other values of $\eta$. For instance, for $\sigma=4$ and $\eta=0.08$, one finds $\Omega\left(B_{1}\right)=\Omega\left(B_{2}\right)=\{2\}$. That is, in this economy the assets yield a liquidity return only in the low-growth state.
} 
In terms of the binding patterns for each parametrization, for $\sigma=8,9,10, \Omega\left(B_{i}\right)=\{1,2\}$ for all $i$ and all values of $\eta$. For $\sigma=7, \Omega\left(B_{i}\right)=\varnothing$ for all $i$, if $\eta=1$, but $\Omega\left(B_{i}\right)=\{1,2\}$ for intermediate values of $\eta$, e.g., ranging from (and including) 0.001 and 0.999 . For $\sigma=6$, the sets $\Omega\left(B_{i}\right)$ of states in which the assets yield a liquidity return depend more subtly on the value of $\eta$. Specifically, assets yield a liquidity return in all states, i.e., $\Omega\left(B_{i}\right)=\{1,2\}$ for all $i$, if $\eta \leq 0.986$. If $\eta$ is larger than 0.986 but smaller than or equal to 0.991 , then $\Omega\left(B_{1}\right)=\Omega\left(B_{2}\right)=\{2\}$, i.e., assets yield a liquidity return only in the low-growth state. Finally, $\Omega\left(B_{i}\right)=\varnothing$ for all $i$, if $\eta$ is larger than 0.991. Similar binding patterns are found for $\sigma=5$ and 4 .

So far I have explored the quantitative implications of varying $\eta$ but only in the context of economies with no exogenous liquidity differences between bonds and equity, i.e., only in economies with $\theta=0$. To conclude, I report how the economy corresponding to the fourth row of Table 4, namely, the baseline parametrization but with $\sigma=4$ and $\theta=0.0222$, behaves as $\eta$ varies.
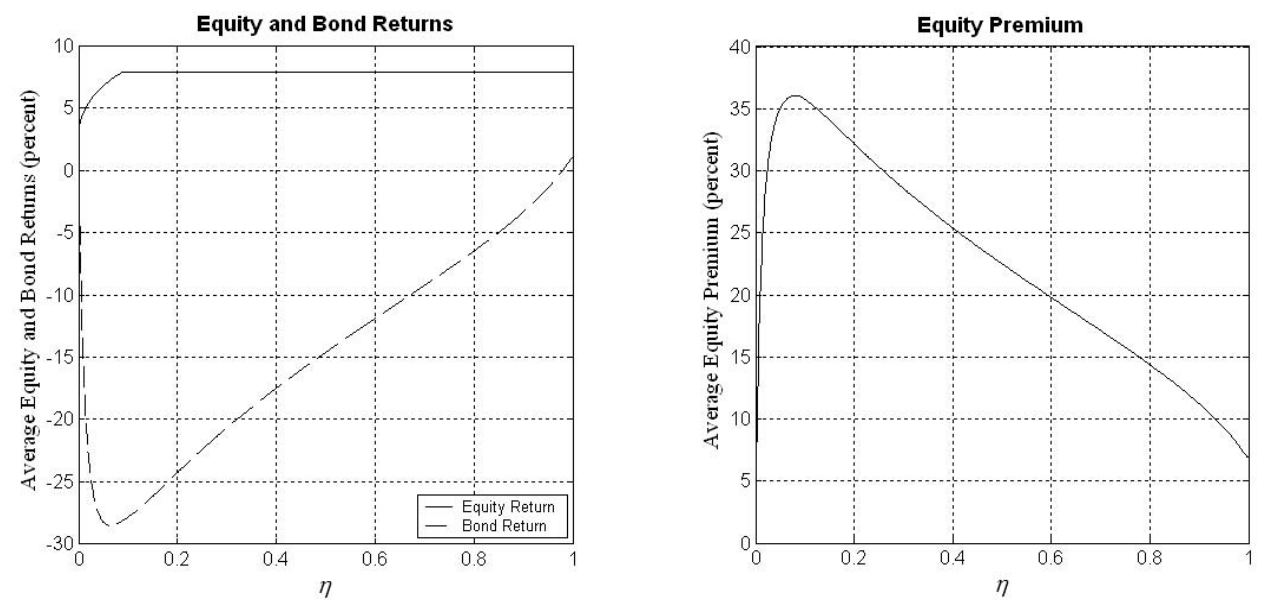

Figure 6: Bargaining power and asset returns for the baseline economy $(\sigma=4$ and $\theta=0.0222)$

The first panel of Figure 6 displays the equity and bond returns, and the second panel the equity premium, all as functions of $\eta$. Note that at $\eta=1$ the bond return is exactly $1 \%$ and the equity premium $6.89 \%$, as is to be expected from the fourth row of Table 4 . Bonds yield a 
liquidity return in all states of the world, i.e., $\Omega_{\theta}\left(B_{i}\right)=\{1,2\}$ for $i=1$ and 2 . Equity yields a liquidity return only for relatively low values of $\eta$, e.g., less than 0.089 , but for higher values, $\Omega\left(B_{i}\right)=\varnothing$ for $i=1$ and 2 . (This is the point at which the equity return becomes flat in the left panel.) Note that depending on the value of $\eta$, the bond return can be extremely low, almost $-30 \%$, and as high as $1 \%$. Accordingly, the equity premium can range from $0.7 \%$ (for $\eta=0$ ) to $36 \%$ (for $\eta=0.075$ ) or $6.89 \%$ (for $\eta=1$ ). From Figure 6 it seems clear that, once again, the equity premia reported in Section 4.2 are - if anything - on the low side of the magnitudes that can be generated by the theory if buyers have some, but not all, the bargaining power in decentralized trades. 